\title{
32. DATA REPORT: STRUCTURAL MEASUREMENTS FROM SITES 894 AND 895, HESS DEEP1
}

\author{
C.J. MacLeod, ${ }^{2}$ C.E. Manning, ${ }^{3}$ F. Boudier, ${ }^{4}$ B. Célérier, ${ }^{4}$ L.A. Kennedy, ${ }^{5}$ P. Kelso,${ }^{6}$ E. Kikawa, ${ }^{7}$ J.E. Pariso,${ }^{8}$ and C. Richter ${ }^{9}$
}

\section{BACKGROUND}

Structural geological studies of the core recovered from Ocean Drilling Program (ODP) Leg 147 are vital in understanding both the geometry of accretion and dynamics of the axial magma chamber(s) beneath the fast-spreading East Pacific Rise and the rifting processes that led to formation of the Hess Deep. Knowledge of the orientation of structures and other physical property measurements in the geographical reference frame is crucial. Unfortunately, however, cores are not normally oriented in azimuth; furthermore, they are typically broken into small, independently-rotated pieces by the drilling process, so that there is no comparative reference between adjacent core pieces. Even dip measurements made relative to the axis of the borehole may not be of significance if the borehole is deviated, and/or tectonic rotation of the section has occurred subsequent to the creation of the structures. Downhole tools that provide the direct orientation of core as it enters the core barrel are in development, but these are expensive, beset by technical problems and hence rarely used (see discussions in MacLeod et al., 1992, 1994).

An alternative technique, used in this paper, is to reorient structural features relative to the stable magnetic remanence directions of demagnetized core samples. By so doing it is assumed that samples from different core pieces acquired their remanences in the same field direction (and slowly, so that the effects of secular variation are averaged out). This technique provides a relative reference, but can only be related to modern geographical coordinates if it can be demonstrated that no tectonic rotations have occurred subsequent to acquisition of the magnetization.

A mechanism for reorienting core without recourse to paleomagnetic data has been developed by MacLeod et al. (1992, 1994). By comparing inclined, planar features in the cores with their direct representations on electrical or acoustic images of the borehole wall, which are themselves reliably oriented in the geographical reference frame, cores can be fully reoriented. Indeed, by orienting core pieces in this way the azimuthal components of stable magnetic remanence vectors can also be restored to geographical coordinates, and tectonic rotations detected. Application of this technique to Hole $894 \mathrm{G}$, the only hole drilled on Leg 147 for which borehole imaging data were

'Mével, C., Gillis, K.M., Allan, J.F., and Meyer, P.S.(Eds.), 1996. Proc. ODP, Sci. Results, 147: College Station, TX (Ocean Drilling Program).

2Department of Earth Sciences, University of Wales College of Cardiff, P.O. Box 914, Cardiff CF1 3YE, United Kingdom. Formerly at: Institute of Oceanographic Sciences, Brook Road, Wormley, Surrey GU8 5UB, United Kingdom; and Borehole Research, Department of Geology, University of Leicester, Leicester LE1 7RH, United Kingdom.macleod@cardiff.ac.uk

${ }^{3}$ Department of Earth and Space Sciences, University of California at Los Angeles, Los Angeles, CA 90024-1567, U.S.A.

${ }^{4}$ ISTEEM, Université de Montpellier II, 34095 Montpellier cedex 05, France.

${ }^{3}$ Center for Tectonophysics, Texas A\&M University, College Station, TX 77843, U.S.A.

${ }^{6}$ Lake Superior State University, Sault Sainte Marie, MI 49783, U.S.A.

Department of Marine Geology. Geological Survey of Japan, 1-1-3 Higashi, Tsukuba, Ibaraki 305, Japan. Current address: Faculty of Education, Toyama University, Toyama, Japan.

'School of Oceanography, University of Washington, Seattle, WA 98195, U.S.A

'Ocean Drilling Program, Texas A\&M University Research Park, 1000 Discovery Drive, College Station, TX 77845, U.S.A. acquired, is made by MacLeod et al. (1995), Célérier et al. (this volume) and MacLeod, Célérier, et al. (this volume).

\section{METHODS}

The fundamental basis of any structural study of borehole cores must be a systematic, self-consistent database that documents the position and (at least relative) orientation of all structural features. The purpose of this data report is to present in an internally consistent format measurements of the orientations of more than 1200 structures at Sites 894 and 895 . Following the procedure described by MacLeod et al. (1992, 1994; summarized in Gillis, Mével, Allan, et al., 1993), the axis of the borehole was considered vertical, and azimuths of structures were measured relative to artificial "core-liner coordinates." "North" was defined as perpendicular to the cut surface and lying in the working half of the core. It is stressed that these "core coordinate" measurements (figures given in italics in Tables 1 and 2) have no geographical significance; the orientations of individual structures are comparable only within contiguous core pieces.

Preliminary, partial restoration of the core fabric measurements to a common reference frame was made by rotating the stable magnetic remanence direction measured in the same contiguous core pieces to the same horizontal direction (magnetic North, assuming normal polarity: MacLeod, Célérier, et al., this volume). This was possible for between one-third and one-half of the measurements made (columns under the heading "Rest D only, strike and dip," shown in bold type in Tables 1 and 2), by using the stable remanence directions from both shipboard and post-cruise samples. The origin of these paleomagnetic measurements is given in the column entitled "Meas. by": $\mathrm{S}=$ shipboard data $\mathrm{C}=$ samples analyzed by $\mathrm{C}$. Richter; $\mathrm{J}=$ samples analyzed by J. Pariso; and P = samples analyzed by P. Kelso.

In this work, a second rotation was also made to bring the measurements to a common magnetic inclination as well as declination (columns under the heading "Rest D + I, strike and dip," shown in bold type in Tables 1 and 2), because inclinations of the stable remanence directions at Site 894 and Hole $895 \mathrm{E}$ are consistently skewed by approximately $35^{\circ}-40^{\circ}$ down toward the declination direction in comparison to the expected field direction for the latitude of the drill site of $+4.6^{\circ}$ (Pariso et al., this volume; Kelso et al., this volume; MacLeod, Célérier, et al., this volume). The disparity in inclinations is most readily interpreted in terms of a tectonic rotation of the drill sites with a horizontal component of this magnitude (Gillis, Mével, Allan, et al., 1993; MacLeod, Célérier, et al., this volume).

By comparing fracture distributions on borehole electrical imagery with the distribution of veins oriented with respect to the stable remanence direction, MacLeod et al. (1995), Célérier et al. (this volume) and MacLeod, Manning, et al. (this volume) have suggested that there has also been a vertical axis component of rotation (estimated at approximately $30^{\circ}$ counterclockwise), such that the stable remanence directions do not point toward present-day north. The "restored" data given in bold type in Tables 1 and 2 are reoriented only with respect to this stable remanence direction and cannot, therefore, be regarded as fully restored to present-day geographical coordinates. Full discussion of these complexities, and interpretation of the data tabulated here, is made elsewhere in specialist papers (e.g., Boudier 
et al., this volume; Célérier et al., this volume; MacLeod et al., 1995; MacLeod, Boudier, et al., this volume; MacLeod, Célérier, et al., this volume: Manning and MacLeod, this volume; Richter et al., this volume).

\section{EXPLANATION OF TABLES}

The depths quoted in Tables 1 and 2, given in meters below seafloor (mbsf), are curated depths (i.e., assuming that all recovered core came from the top of the drilled interval). "Lith" = lithology, with (in Table 1) $\mathrm{G}=$ gabbro, $\mathrm{OG}=$ olivine gabbro, $\mathrm{B}=$ basalt (dike rock), $\mathrm{GN}$ = gabbronorite, and OGN = olivine gabbronorite. Unit $=$ lithostratigraphic unit, defined in Gillis, Mével, Allan, et al. (1993). Abbreviations of feature types (Table 1) are as follows: fol cataclas = foliated cataclasite; ultracataclas = ultracataclasite; fault brecc $=$ fault breccia; fels dike $=$ felsic dikelet; and dike $\operatorname{marg}=$ chilled dike margin. Foliations in Site 894 rocks are defined by the alignment of anisometric plagioclase crystals, and are interpreted by MacLeod, Boudier, et al. (this volume) as magmatic flow fabrics. Vein fill (Table 1) is as defined in Manning and MacLeod (this volume) and Manning et al. (this volume): $\mathrm{A}=$ amphibole vein type; $\mathrm{C}=$ chlorite vein type $( \pm$ calc-silicates); $\mathrm{S}=$ chlorite-smectite vein type (i.e., \pm clay); and $\mathrm{Z}=$ zeolite-calcite vein type. Lithologies in Table 2 are as follows: $\mathrm{DN}=$ dunite; IMP DN = dunite with interstitial plagioclase \pm clinopyroxene "impregnations"; $\mathrm{HZ}=$ harzburgite; TROCT = troctolite; ROD G = altered ("rodingitized") gabbro; $\mathrm{G}=$ gabbro; $\mathrm{DN} / \mathrm{HZ}=$ planar contact between harzburgite and dunite. Feature types (Table 2) are as follows: vein = vein, usually of serpentine (sensu lato), however, no systematic subdivision of vein type by mineralogy was undertaken in this study; vein netwk = network of parallel, very fine serpentine veins; ign contact $=$ igneous contact $;$ plag impr $=$ planar impregnation of plagioclase; foln in $\mathrm{G}=$ foliation in mafic rocks. Foliations in harzburgites and dunites are interpreted by Boudier et al. (this volume) as high-temperature, solid-state deformation fabrics.

Combined strikes (str) and dips (dip) of planar structural features are reconstituted from two apparent dip measurements, with or with- out a direct strike measurement. Direction of dip is $90^{\circ}$ clockwise from the strike direction in all cases. Both the combined structural measurements and the stable magnetic remanence directions $(\mathrm{dec}=$ declination, and inc = inclination of magnetic vector) are given in synthetic "core-liner coordinates" (shown in italics in Tables 1 and 2), discussed above.

Spatially reoriented measurements are shown in bold in Tables 1 and 2. In the columns "Rest (D only)" structural measurements have been rotated so that the horizontal component (declination) of the stable magnetic remanence direction points towards north. In columns "Rest D + I" structural measurements have been restored in inclination, too, such that the measured stable remanence declination is set to "North" $\left(000^{\circ}\right)$ and inclination to $+4.6^{\circ}$.

\section{REFERENCES}

Gillis, K., Mével, C., Allan, J., et al., 1993. Proc. ODP, Init. Repts., 147: College Station, TX (Ocean Drilling Program).

MacLeod, C.J., Célérier, B., and Harvey, P.K., 1995. Further techniques for core reorientation by core-log integration: application to structural studies of the lower oceanic crust in Hess Deep, eastern Pacific. Scientific Drilling, 5:77-86.

MacLeod, C.J., Parson, L.M., and Sager, W.W., 1994. Reorientation of cores using the Formation MicroScanner and Borehole Televiewer: application to structural and paleomagnetic studies with the Ocean Drilling Program. In Hawkins, J., Parson, L., Allan, J., et al., Proc. ODP, Sci. Results, 135: College Station, TX (Ocean Drilling Program), 301-311.

MacLeod, C.J., Parson, L.M., Sager, W.W., and the ODP Leg 135 Scientific Party, 1992. Identification of tectonic rotations in boreholes by the integration of core information with Formation MicroScanner and Borehole Televiewer images. In Hurst, A., Griffiths, C.M., and Worthington, P.F. (Eds.), Geological Applications of Wireline Logs II. Geol. Soc. Spec. Publ. London, 65:235-246.

\author{
Date of initial receipt: 8 August 1994 \\ Date of acceptance: 24 February 1995 \\ Ms 147SR-034
}

Table 1. Structural data from Site 894.

\begin{tabular}{|c|c|c|c|c|c|c|c|c|c|c|c|c|c|c|c|}
\hline \multirow[b]{2}{*}{ Sample number } & \multirow{2}{*}{$\begin{array}{l}\text { Depth } \\
\text { (mbsf) }\end{array}$} & \multirow[b]{2}{*}{ Lith. } & \multirow[b]{2}{*}{ Unit } & \multirow[b]{2}{*}{ Feature type } & \multirow{2}{*}{$\begin{array}{l}\text { Vein } \\
\text { fill }\end{array}$} & \multirow[b]{2}{*}{ Feat. \# } & \multicolumn{2}{|c|}{ Combined } & \multicolumn{2}{|c|}{ Stable mag. rem. } & \multirow{2}{*}{$\begin{array}{c}\text { Meas. } \\
\text { by }\end{array}$} & \multicolumn{2}{|c|}{ Rest (D only) } & \multicolumn{2}{|c|}{ Rest (D + I) } \\
\hline & & & & & & & $\operatorname{str}\left({ }^{\circ}\right)$ & $\operatorname{dip}\left({ }^{\circ}\right)$ & $\operatorname{dec}\left({ }^{\circ}\right)$ & $\operatorname{inc}\left({ }^{\circ}\right)$ & & $\operatorname{str}\left({ }^{\circ}\right)$ & $\operatorname{dip}\left({ }^{\circ}\right)$ & $\operatorname{str}\left({ }^{\circ}\right)$ & $\operatorname{dip}\left({ }^{\circ}\right)$ \\
\hline $\begin{array}{l}\text { Hole 894E- } \\
\quad 3 \mathrm{R}-1,69 \mathrm{~cm} \text { (Piece 10) }\end{array}$ & 19.59 & G & & vein & & 1 & 191 & 82 & & & & & & & \\
\hline $\begin{array}{l}\text { Hole 894F- } \\
\text { IR-1, } 5 \mathrm{~cm} \text { (Piece 1) } \\
\text { 2R-1, } 7 \mathrm{~cm} \text { (Piece 1) } \\
\text { 2R-1, } 16 \mathrm{~cm} \text { (Piece2) } \\
2 \mathrm{R}-1,22 \mathrm{~cm} \text { (Piece 2) } \\
2 \mathrm{R}-1,30 \mathrm{~cm} \text { (Piece 3) } \\
2 \mathrm{R}-1,29 \mathrm{~cm} \text { (Piece 3) } \\
2 \mathrm{R}-1,33 \mathrm{~cm} \text { (Piece 3) } \\
\text { 2R-1, } 56 \mathrm{~cm} \text { (Piece 7) } \\
3 \mathrm{R}-1,19 \mathrm{~cm} \text { (Piece 4) } \\
\text { 3R-1, } 37 \mathrm{~cm} \text { (Piece 6) } \\
\text { 3R-1, } 54 \mathrm{~cm} \text { (Piece 9) }\end{array}$ & $\begin{array}{r}0.05 \\
9.37 \\
9.46 \\
9.52 \\
9.60 \\
9.59 \\
9.63 \\
9.86 \\
19.09 \\
19.27 \\
19.44\end{array}$ & $\begin{array}{l}\text { G } \\
\text { G } \\
\text { G } \\
\text { G } \\
\text { G } \\
\text { G } \\
\text { G } \\
\text { G } \\
\text { G } \\
\text { G } \\
\text { G }\end{array}$ & $\begin{array}{l}1 \\
1 \\
1 \\
1 \\
1 \\
1 \\
1 \\
1 \\
1 \\
1 \\
1 \\
1\end{array}$ & $\begin{array}{l}\text { fol catacl } \\
\text { fault } \\
\text { vein } \\
\text { vein } \\
\text { fault } \\
\text { fault } \\
\text { cataclasite } \\
\text { ultraclasite } \\
\text { ultracatacl } \\
\text { ultracatacl } \\
\text { ultracatacl }\end{array}$ & & $\begin{array}{l}1 \\
1 \\
1 \\
2 \\
1 \\
1 \\
2 \\
3 \\
1 \\
1 \\
1 \\
1\end{array}$ & $\begin{array}{l}000 \\
349 \\
144 \\
154 \\
019 \\
177 \\
247 \\
168 \\
220\end{array}$ & $\begin{array}{l}70 \\
85 \\
68 \\
57 \\
83 \\
59 \\
40 \\
31 \\
42\end{array}$ & & & & & & & \\
\hline $\begin{array}{l}\text { Hole 894G- } \\
\text { IR-1, } 11 \mathrm{~cm} \text { (Piece 2) } \\
\text { IR-1, } 12 \mathrm{~cm} \text { (Piece 2) } \\
\text { 2R-1, } 18 \mathrm{~cm} \text { (Piece 3) } \\
\text { 2R-1, } 37 \mathrm{~cm} \text { (Piece 5) } \\
\text { 2R-1, } 41 \mathrm{~cm} \text { (Piece 5) } \\
\text { 2R-1, } 60 \mathrm{~cm} \text { (Piece 9) } \\
2 \mathrm{R}-1,64 \mathrm{~cm} \text { (Piece 9) } \\
2 \mathrm{R}-1,67 \mathrm{~cm} \text { (Piece 9) } \\
\text { 2R-1, } 120 \mathrm{~cm} \text { (Piece 15) } \\
\text { 2R-1, } 114 \mathrm{~cm} \text { (Piece 15) } \\
\text { 2R-1, } 116 \mathrm{~cm} \text { (Piece 15) } \\
\text { 2R-1, } 143 \mathrm{~cm} \text { (Piece 18) } \\
\text { 2R-1, } 138 \mathrm{~cm} \text { (Piece 18) } \\
\text { 2R-2, } 13 \mathrm{~cm} \text { (Piece 3) } \\
\text { 2R-2, } 14 \mathrm{~cm} \text { (Piece 3) } \\
\text { 2R-2, } 32 \mathrm{~cm} \text { (Piece 7) } \\
\text { 2R-2, } 32 \mathrm{~cm} \text { (Piece 7) }\end{array}$ & $\begin{array}{l}18.71 \\
18.72 \\
28.78 \\
28.97 \\
29.01 \\
29.20 \\
29.24 \\
29.27 \\
29.80 \\
29.74 \\
29.76 \\
30.03 \\
29.98 \\
30.26 \\
30.27 \\
30.45 \\
30.45\end{array}$ & $\begin{array}{l}\text { OG } \\
\text { OG } \\
B \\
\text { B } \\
\text { B } \\
\text { B } \\
\text { B } \\
\text { B } \\
\text { B } \\
\text { B } \\
\text { B } \\
\text { B } \\
\text { B } \\
\text { B } \\
\text { B } \\
\text { B } \\
\text { B }\end{array}$ & $\begin{array}{l}1 \\
1 \\
2 \\
2 \\
2 \\
2 \\
2 \\
2 \\
2 \\
2 \\
2 \\
2 \\
2 \\
2 \\
2 \\
2 \\
2\end{array}$ & $\begin{array}{l}\text { vein } \\
\text { vein } \\
\text { vein } \\
\text { vein } \\
\text { vein } \\
\text { vein } \\
\text { vein } \\
\text { vein } \\
\text { vein } \\
\text { vein } \\
\text { vein } \\
\text { vein } \\
\text { vein } \\
\text { vein } \\
\text { vein } \\
\text { vein } \\
\text { vein }\end{array}$ & $\begin{array}{l}S \\
S \\
S \\
S \\
S \\
S \\
S \\
Z \\
S \\
S \\
S \\
S \\
S \\
S \\
S \\
S \\
S\end{array}$ & $\begin{array}{l}1 \\
2 \\
1 \\
1 \\
2 \\
1 \\
2 \\
3 \\
1 \\
2 \\
3 \\
1 \\
2 \\
1 \\
2 \\
2 \\
3\end{array}$ & $\begin{array}{l}260 \\
240 \\
356 \\
346 \\
168 \\
305 \\
274 \\
070 \\
190 \\
184 \\
331 \\
149 \\
003 \\
002 \\
233 \\
217 \\
205\end{array}$ & $\begin{array}{r}38 \\
25 \\
57 \\
51 \\
61 \\
64 \\
42 \\
54 \\
65 \\
81 \\
81 \\
62 \\
18 \\
86 \\
40 \\
5 \\
48\end{array}$ & $\begin{array}{l}074.9 \\
074.9 \\
074.9 \\
145.0 \\
145.0 \\
145.0\end{array}$ & $\begin{array}{l}26.8 \\
26.8 \\
26.8 \\
21 \\
21 \\
21\end{array}$ & $\begin{array}{l}\mathrm{S} \\
\mathrm{S} \\
\mathrm{S} \\
\mathrm{P} \\
\mathrm{P} \\
\mathrm{P}\end{array}$ & $\begin{array}{l}230 \\
199 \\
355 \\
045 \\
039 \\
186\end{array}$ & $\begin{array}{l}64 \\
42 \\
54 \\
65 \\
81 \\
81\end{array}$ & $\begin{array}{l}154.5 \\
\\
219.2 \\
172.9 \\
011.0 \\
048.9 \\
219.8 \\
183.2\end{array}$ & $\begin{array}{l}\mathbf{4 8 . 2} \\
\mathbf{3 9 . 6} \\
\mathbf{5 5 . 2} \\
\mathbf{7 7 . 0} \\
\mathbf{8 8 . 5} \\
\mathbf{7 9 . 7}\end{array}$ \\
\hline
\end{tabular}


Table 1 (continued).

\begin{tabular}{|c|c|c|c|c|c|c|c|c|}
\hline & Depth & & & & Vein & & Con & ined \\
\hline Sample number & (mbsf) & Lith. & Unit & Feature type & fill & Feat. \# & $\operatorname{str}\left({ }^{\circ}\right)$ & $\operatorname{dip}\left({ }^{\circ}\right)$ \\
\hline 2R-2, $40 \mathrm{~cm}$ (Piece 7) & 30.53 & B & 2 & vein & C & 4 & 257 & 83 \\
\hline $2 \mathrm{R}-2,30 \mathrm{~cm}$ (Piece 7) & 30.43 & B & 2 & vein & $S$ & 5 & 347 & 48 \\
\hline $2 \mathrm{R}-2,54 \mathrm{~cm}$ (Piece 10) & 30.67 & B & 2 & vein & $\mathrm{S}$ & 1 & 323 & 5 \\
\hline 2R-2, $58 \mathrm{~cm}$ (Piece 10) & 30.71 & B & 2 & vein & $\mathrm{S}$ & 2 & 292 & 5 \\
\hline 2R-2, $64 \mathrm{~cm}$ (Piece 10) & 30.77 & B & 2 & vein & C & 3 & 041 & 29 \\
\hline $2 \mathrm{R}-2,65 \mathrm{~cm}$ (Piece 10) & 30.78 & B & 2 & vein & $\mathrm{Z}$ & 4 & 169 & 71 \\
\hline 2R-2, $73 \mathrm{~cm}$ (Piece 11) & 30.86 & B & 2 & vein & $\mathrm{S}$ & $i$ & 334 & 65 \\
\hline $2 \mathrm{R}-2,67 \mathrm{~cm}$ (Piece 11) & 30.80 & B & 2 & vein & $\mathrm{S}$ & 2 & 023 & 55 \\
\hline $2 \mathrm{R}-2,74 \mathrm{~cm}$ (Piece 11) & 30.87 & B & 2 & vein & $\mathrm{S}$ & 5 & 356 & 69 \\
\hline $2 \mathrm{R}-2,73 \mathrm{~cm}$ (Piece 11) & 30.86 & B & 2 & vein & C & 6 & 220 & 47 \\
\hline $2 \mathrm{R}-2,76 \mathrm{~cm}$ (Piece 11 ) & 30.89 & B & 2 & vein & $\mathrm{S}$ & 8 & 147 & 25 \\
\hline $2 \mathrm{R}-2,102 \mathrm{~cm}$ (Piece 15) & 31.15 & B & 2 & vein & $\mathrm{C}$ & 1 & 108 & 24 \\
\hline $2 \mathrm{R}-2,107 \mathrm{~cm}$ (Piece 15) & 31.20 & B & 2 & vein & C & 2 & 095 & 36 \\
\hline $2 \mathrm{R}-2,98 \mathrm{~cm}$ (Piece 15) & 31.11 & B & 2 & vein & $\mathrm{C}$ & 3 & 154 & 18 \\
\hline 2R-2, $95 \mathrm{~cm}$ (Piece 15) & 31.08 & B & 2 & vein & $\mathrm{C}$ & 4 & 146 & 79 \\
\hline $2 \mathrm{R}-2,122 \mathrm{~cm}$ (Piece 17) & 31.35 & B & 2 & vein & $\mathrm{S}$ & 1 & 005 & 43 \\
\hline $2 \mathrm{R}-2,141 \mathrm{~cm}$ (Piece 19) & 31.54 & OG & 3 & vein & $\mathrm{s}$ & 1 & 056 & 63 \\
\hline 2R-2, $137 \mathrm{~cm}$ (Piece 19) & 31.50 & OG & 3 & vein & $\mathrm{S}$ & 2 & 011 & 36 \\
\hline 2R-2, $146 \mathrm{~cm}$ (Piece 19) & 31.59 & OG & 3 & fault & $\mathrm{S}$ & 3 & 010 & 64 \\
\hline 2R-2, $138 \mathrm{~cm}$ (Piece 19) & 31.51 & OG & 3 & vein & S & 4 & 129 & 57 \\
\hline $2 \mathrm{R}-3,15 \mathrm{~cm}$ (Piece 3$)$ & 31.75 & OG & 3 & vein & $\mathrm{C}$ & 1 & 232 & 25 \\
\hline $2 \mathrm{R}-3,16.5 \mathrm{~cm}$ (Piece 3$)$ & 31.77 & OG & 3 & vein & $\mathrm{s}$ & 2 & 118 & 53 \\
\hline $2 \mathrm{R}-3,17.5 \mathrm{~cm}$ (Piece 3 ) & 31.78 & OG & 3 & vein & $\mathrm{C}$ & 3 & 168 & 43 \\
\hline $2 \mathrm{R}-3,18 \mathrm{~cm}$ (Piece 3$)$ & 31.78 & OG & 3 & vein & $\mathrm{C}$ & 4 & 140 & So \\
\hline $2 \mathrm{R}-3,18 \mathrm{~cm}$ (Piece 3$)$ & 31.78 & OG & 3 & vein & C & 6 & 324 & 52 \\
\hline 2R-3, $19 \mathrm{~cm}$ (Piece 3) & 31.79 & OG & 3 & vein & $\mathrm{C}$ & 7 & 320 & 64 \\
\hline $2 \mathrm{R}-3,26.5 \mathrm{~cm}$ (Piece 4) & 31.87 & OG & 3 & vein & $\mathrm{C}$ & i & 005 & 23 \\
\hline $2 \mathrm{R}-3,26 \mathrm{~cm}$ (Piece 4) & 31.86 & OG & 3 & vein & C & 2 & 064 & 22 \\
\hline 2R-3, $42.5 \mathrm{~cm}$ (Piece 5) & 32.03 & OG & 3 & vein & C & 1 & 000 & 60 \\
\hline 2R-3, $39 \mathrm{~cm}$ (Piece 5) & 31.99 & OG & 3 & fol catacl & $\mathrm{C}$ & 2 & 144 & 85 \\
\hline 2R-3, $53 \mathrm{~cm}$ (Piece 6) & 32.13 & OG & 3 & fol catacl & $\mathrm{C}$ & $i$ & 354 & 90 \\
\hline $2 \mathrm{R}-3,55 \mathrm{~cm}$ (Piece 6) & 32.15 & OG & 3 & vein & $\mathrm{C}$ & 2 & $2 / 4$ & 43 \\
\hline 2R $-3,53 \mathrm{~cm}$ (Piece 6) & 32.13 & OG & 3 & vein & $\mathrm{C}$ & 3 & 158 & 81 \\
\hline $2 \mathrm{R}-3,82 \mathrm{~cm}$ (Piece 11) & 32.42 & OG & 3 & fol catacl & $\mathrm{C}$ & 1 & 168 & 90 \\
\hline $2 \mathrm{R}-3,80 \mathrm{~cm}$ (Piece 11 ) & 32.40 & OG & 3 & vein & $\mathrm{C}$ & 2 & 194 & 85 \\
\hline $2 \mathrm{R}-3,80 \mathrm{~cm}$ (Piece 11) & 32.40 & OG & 3 & vein & $\mathrm{C}$ & 3 & 268 & 44 \\
\hline $2 \mathrm{R}-3,94 \mathrm{~cm}$ (Piece 12) & 32.54 & OG & 3 & fol catacl & $\mathrm{C}$ & 1 & 004 & 90 \\
\hline $2 \mathrm{R}-3,82 \mathrm{~cm}$ (Piece 12) & 32.42 & OG & 3 & vein & $\mathrm{C}$ & 2 & 182 & 67 \\
\hline 2R-3, $110 \mathrm{~cm}$ (Piece 14) & 32.70 & OG & 3 & vein & $\mathrm{s}$ & 1 & 292 & 35 \\
\hline 2R-3, $112 \mathrm{~cm}$ (Piece 14) & 32.72 & OG & 3 & vein & C & 2 & 288 & 9 \\
\hline $2 \mathrm{R}-3,114.5 \mathrm{~cm}$ (Piece 14) & 32.75 & OG & 3 & vein & $\mathrm{S}$ & 3 & 247 & 43 \\
\hline $4 \mathrm{R}-1,36 \mathrm{~cm}$ (Piece 7) & 45.36 & GN & 4 & vein & S & 1 & 300 & 80 \\
\hline $4 \mathrm{R}-1,36 \mathrm{~cm}$ (Piece 7) & 45.36 & GN & 4 & vein & S & 2 & 159 & 26 \\
\hline $4 \mathrm{R}-1,72 \mathrm{~cm}$ (Piece 12) & 45.72 & GN & 4 & vein & $\mathrm{C}$ & 1 & 008 & 50 \\
\hline $4 \mathrm{R}-1,77 \mathrm{~cm}$ (Piece 12) & 45.77 & GN & 4 & vein & $\mathrm{s}$ & 2 & 340 & 52 \\
\hline $4 \mathrm{R}-1,72 \mathrm{~cm}$ (Piece 12) & 45.72 & GN & 4 & vein & $\mathrm{S}$ & 3 & 134 & 52 \\
\hline $4 \mathrm{R}-1,74 \mathrm{~cm}$ (Piece 12$)$ & 45.74 & GN & 4 & vein & $\mathrm{s}$ & 4 & 177 & 57 \\
\hline $4 \mathrm{R}-1,73 \mathrm{~cm}$ (Piece 12) & 45.73 & GN & 4 & vein & S & 5 & 330 & 49 \\
\hline $4 \mathrm{R}-1,78 \mathrm{~cm}$ (Piece 12) & 45.78 & GN & 4 & vein & S & 6 & 178 & 62 \\
\hline $4 \mathrm{R}-1,78 \mathrm{~cm}$ (Piece 12) & 45.78 & GN & 4 & vein & S & 7 & 088 & 90 \\
\hline $4 \mathrm{R}-1,83 \mathrm{~cm}$ (Piece 13 ) & 45.83 & $\mathrm{GN}$ & 4 & vein & S & 1 & 093 & 57 \\
\hline $4 \mathrm{R}-1,85 \mathrm{~cm}$ (Piece 13) & 45.85 & $\mathrm{GN}$ & 4 & vein & $\mathrm{s}$ & 2 & 052 & 45 \\
\hline $4 \mathrm{R}-1,90 \mathrm{~cm}$ (Piece 13) & 45.90 & GN & 4 & vein & S & 3 & 357 & 81 \\
\hline $4 \mathrm{R}-1,115 \mathrm{~cm}$ (Piece 16) & 46.15 & $\mathrm{GN}$ & 4 & vein & A & i & 190 & 70 \\
\hline $4 \mathrm{R}-1,115 \mathrm{~cm}$ (Piece 16) & 46.15 & GN & 4 & foliation & & 2 & 318 & 46 \\
\hline $4 \mathrm{R}-2,35 \mathrm{~cm}$ (Piece 7 ) & 46.69 & GN & 4 & vein & $\mathrm{S}$ & 1 & 149 & 81 \\
\hline $4 \mathrm{R}-2.33 \mathrm{~cm}$ (Piece 7) & 46.67 & GN & 4 & vein & s & 2 & 288 & 53 \\
\hline $4 \mathrm{R}-2,34 \mathrm{~cm}$ (Piece 7) & 46.68 & $\mathrm{GN}$ & 4 & vein & S & 3 & 002 & 86 \\
\hline $4 \mathrm{R}-2,39 \mathrm{~cm}$ (Piece 7) & 46.73 & GN & 4 & vein & S & 4 & 223 & 43 \\
\hline $4 \mathrm{R}-2,36 \mathrm{~cm}$ (Piece 7) & 46.70 & GN & 4 & vein & $\mathrm{A}$ & 5 & 083 & 68 \\
\hline $4 \mathrm{R}-2,54 \mathrm{~cm}$ (Piece 9) & 46.88 & GN & 4 & vein & $\mathrm{S}$ & i & 324 & 56 \\
\hline 4R-2, $57 \mathrm{~cm}$ (Piece 9) & 46.91 & GN & 4 & vein & $\mathrm{s}$ & 2 & 323 & 52 \\
\hline $4 \mathrm{R}-2,63 \mathrm{~cm}$ (Piece 9) & 46.97 & GN & 4 & vein & $\mathrm{S}$ & 3 & 316 & 49 \\
\hline $4 \mathrm{R}-2,49 \mathrm{~cm}$ (Piece 9) & 46.83 & GN & 4 & vein & S & 4 & 337 & 55 \\
\hline $4 \mathrm{R}-2,54 \mathrm{~cm}$ (Piece 9) & 46.88 & GN & 4 & vein & S & 5 & 120 & 84 \\
\hline $4 \mathrm{R}-2,78 \mathrm{~cm}$ (Piece 10) & 47.12 & GN & 4 & vein & $\mathrm{s}$ & 1 & 255 & 31 \\
\hline $4 \mathrm{R}-2,73 \mathrm{~cm}$ (Piece 10) & 47.07 & GN & 4 & foliation & & 3 & 270 & 55 \\
\hline $4 \mathrm{R}-2,92 \mathrm{~cm}$ (Piece 11) & 47.26 & $\mathrm{GN}$ & 4 & vein & s & 1 & 012 & 76 \\
\hline $4 \mathrm{R}-2,115 \mathrm{~cm}$ (Piece 14) & 47.49 & GN & 4 & vein & S & 1 & 000 & 89 \\
\hline $4 \mathrm{R}-2,116 \mathrm{~cm}$ (Piece 14) & 47.50 & GN & 4 & vein & S & 2 & 000 & 0 \\
\hline $4 \mathrm{R}-2,120 \mathrm{~cm}$ (Piece 14) & 47.54 & GN & 4 & vein & S & 3 & 000 & 0 \\
\hline 6R-1. $16 \mathrm{~cm}$ (Piece 4) & 54.96 & GN & 6 & vein & $\mathrm{C}$ & 1 & 312 & 56 \\
\hline $6 \mathrm{R}-1,18 \mathrm{~cm}$ (Piece 4) & 54.98 & GN & 6 & vein & C & 2 & 326 & 40 \\
\hline $6 \mathrm{R}-1,20 \mathrm{~cm}$ (Piece 4) & 55.00 & GN & 6 & vein & A & 3 & 111 & 68 \\
\hline $6 \mathrm{R}-1,17 \mathrm{~cm}$ (Piece 4) & 54.97 & GN & 6 & vein & $\mathrm{s}$ & 4 & 203 & 72 \\
\hline $6 \mathrm{R}-1,21 \mathrm{~cm}$ (Piece 4) & 55.01 & GN & 6 & vein & $\mathrm{C}$ & 5 & 335 & 42 \\
\hline $6 \mathrm{R}-1,23 \mathrm{~cm}$ (Piece 4) & 55.03 & GN & 6 & vein & $\mathrm{C}$ & 6 & 328 & 40 \\
\hline $6 \mathrm{R}-1.29 \mathrm{~cm}$ (Piece 4) & 55.09 & GN & 6 & vein & $\mathrm{s}$ & 7 & 020 & 46 \\
\hline $6 \mathrm{R}-1,39 \mathrm{~cm}$ (Piece 4) & 55.19 & GN & 6 & vein & A & 8 & 154 & 71 \\
\hline $6 \mathrm{R}-1,31 \mathrm{~cm}$ (Picce 4) & 55.11 & GN & 6 & vein & $\mathrm{C}$ & 9 & 346 & 41 \\
\hline $6 \mathrm{R}-1,40 \mathrm{~cm}$ (Piece 4) & 55.20 & GN & 6 & vein & $\mathrm{C}$ & 14 & 262 & 42 \\
\hline $6 \mathrm{R}-1,40 \mathrm{~cm}$ (Piece 4) & 55.20 & $\mathrm{GN}$ & 6 & vein & $\mathrm{C}$ & 16 & 092 & 39 \\
\hline $6 \mathrm{R}-1,46 \mathrm{~cm}$ (Piece 5) & 55.26 & GN & 6 & vein & C & 1 & 154 & 58 \\
\hline $6 \mathrm{R}-1,47 \mathrm{~cm}$ (Piece 5) & 55.27 & GN & 6 & vein & $\mathrm{C}$ & 3 & 183 & 42 \\
\hline 6R-1, $49 \mathrm{~cm}$ (Piece 5) & 55.29 & $\mathrm{GN}$ & 6 & vein & $\mathrm{C}$ & 4 & 205 & 48 \\
\hline $6 \mathrm{R}-1,45 \mathrm{~cm}$ (Piece 5) & 55.25 & GN & 6 & vein & $\mathrm{S}$ & 5 & 175 & 43 \\
\hline $6 \mathrm{R}-1,58 \mathrm{~cm}$ (Piece 5 ) & 55.38 & GN & 6 & vein & $\mathrm{C}$ & 6 & 350 & 32 \\
\hline $6 \mathrm{R}-1,57 \mathrm{~cm}$ (Piece 5) & 55.37 & $\mathrm{GN}$ & 6 & vein & $\mathrm{C}$ & 7 & 199 & 64 \\
\hline
\end{tabular}


Table 1 (continued).

\begin{tabular}{|c|c|c|c|c|c|c|c|c|}
\hline & Depth & & & & Vein & & Con & pined \\
\hline Sample number & (mbsf) & Lith. & Unit & Feature type & fill & Feat. \# & $\operatorname{str}\left({ }^{\circ}\right)$ & $\operatorname{dip}\left({ }^{\circ}\right)$ \\
\hline 6R-1, $57 \mathrm{~cm}$ (Piece 5) & 55.37 & $\mathrm{GN}$ & 6 & vein & A & 8 & 175 & 70 \\
\hline 6R-1, $62 \mathrm{~cm}$ (Piece 5) & 55.42 & GN & 6 & vein & C & 9 & 173 & 48 \\
\hline $6 \mathrm{R}-1,63 \mathrm{~cm}$ (Piece 5) & 55.43 & GN & 6 & vein & $\mathrm{S}$ & 10 & 049 & 75 \\
\hline $6 \mathrm{R}-1,74 \mathrm{~cm}$ (Piece 7) & 55.54 & GN & 6 & vein & $\mathrm{C}$ & 1 & 246 & 33 \\
\hline 6R-1. $78 \mathrm{~cm}$ (Piece 7) & 55.58 & $\mathrm{GN}$ & 6 & vein & C & 3 & 208 & 33 \\
\hline 6R-1, $73 \mathrm{~cm}$ (Piece 7) & 55.53 & GN & 6 & vein & $\mathrm{S}$ & 5 & 181 & 50 \\
\hline $6 \mathrm{R}-1,96 \mathrm{~cm}$ (Piece 8) & 55.76 & GN & 6 & vein & S & 1 & 163 & 63 \\
\hline $6 \mathrm{R}-1,91 \mathrm{~cm}$ (Piece 8) & 55.71 & GN & 6 & vein & A & 3 & 237 & 18 \\
\hline 6R-1, $87 \mathrm{~cm}$ (Piece 8) & 55.67 & GN & 6 & vein & $\mathrm{S}$ & 4 & 182 & 48 \\
\hline 6R-1, $98 \mathrm{~cm}$ (Piece 8) & 55.78 & GN & 6 & vein & $\mathrm{S}$ & 2 & 180 & 44 \\
\hline $6 \mathrm{R}-1,99 \mathrm{~cm}$ (Piece 8) & 55.79 & GN & 6 & vein & $\mathrm{S}$ & 5 & 056 & 87 \\
\hline $6 \mathrm{R}-1,116 \mathrm{~cm}$ (Piece 10) & 55.96 & GN & 6 & vein & C & 1 & 221 & 19 \\
\hline $6 \mathrm{R}-1,118 \mathrm{~cm}$ (Piece 10) & 55.98 & GN & 6 & vein & C & 2 & 170 & 90 \\
\hline $6 \mathrm{R}-1,121 \mathrm{~cm}$ (Piece 10) & 56.01 & GN & 6 & vein & $\mathrm{S}$ & 3 & 345 & 75 \\
\hline $6 \mathrm{R}-1,138 \mathrm{~cm}$ (Piece 12) & 56.18 & GN & 6 & vein & C & 1 & 198 & 42 \\
\hline $6 \mathrm{R}-1,135 \mathrm{~cm}$ (Piece 12) & 56.15 & $\mathrm{GN}$ & 6 & vein & $\mathrm{C}$ & 2 & 176 & 82 \\
\hline $6 \mathrm{R}-1,142 \mathrm{~cm}$ (Piece 12) & 56.22 & GN & 6 & vein & C & 3 & 217 & 36 \\
\hline $6 \mathrm{R}-1,134 \mathrm{~cm}$ (Piece 12) & 56.14 & $\mathrm{GN}$ & 6 & vein & $\mathrm{S}$ & 4 & 255 & 66 \\
\hline $6 \mathrm{R}-1,136 \mathrm{~cm}$ (Piece 12) & 56.16 & GN & 6 & vein & A & 5 & 005 & 85 \\
\hline 6R-2, $24 \mathrm{~cm}$ (Piece 1) & 56.47 & GN & 6 & vein & $\mathrm{S}$ & 1 & 171 & 50 \\
\hline $6 \mathrm{R}-2,23 \mathrm{~cm}$ (Piece 1) & 56.46 & $\mathrm{GN}$ & 6 & vein & $\mathrm{S}$ & 2 & 385 & 48 \\
\hline $6 \mathrm{R}-2,20 \mathrm{~cm}$ (Piece 1) & 56.43 & GN & 6 & vein & $\mathrm{C}$ & 3 & 182 & 50 \\
\hline $6 \mathrm{R}-2,17 \mathrm{~cm}$ (Piece 1) & 56.40 & GN & 6 & vein & C & 4 & 192 & 41 \\
\hline $6 \mathrm{R}-2,16 \mathrm{~cm}$ (Piece 1) & 56.39 & $\mathrm{GN}$ & 6 & vein & $\mathrm{C}$ & 5 & 178 & 64 \\
\hline $6 \mathrm{R}-2,1 \mathrm{~cm}$ (Piece 1) & 56.24 & GN & 6 & vein & C & 7 & 132 & 26 \\
\hline $6 \mathrm{R}-2,28 \mathrm{~cm}$ (Piece 2) & 56.51 & GN & 6 & vein & C & 1 & 353 & 42 \\
\hline $6 \mathrm{R}-2,29 \mathrm{~cm}$ (Piece 2) & 56.52 & GN & 6 & vein & C & 3 & 303 & 69 \\
\hline $6 \mathrm{R}-2,30 \mathrm{~cm}$ (Piece 2) & 56.53 & GN & 6 & vein & $S$ & 4 & 082 & 75 \\
\hline $6 \mathrm{R}-2,40 \mathrm{~cm}$ (Piece 4) & 56.63 & GN & 6 & ign contact & & 1 & 204 & 7 \\
\hline $6 \mathrm{R}-2,45 \mathrm{~cm}$ (Piece 4) & 56.68 & GN & 6 & vein & C & 2 & 188 & 50 \\
\hline $6 \mathrm{R}-2,46 \mathrm{~cm}$ (Piece 4) & 56.69 & GN & 6 & vein & C & 3 & 170 & 48 \\
\hline $6 \mathrm{R}-2,58 \mathrm{~cm}$ (Piece 5) & 56.81 & GN & 6 & vein & $\mathrm{S}$ & 1 & 346 & 49 \\
\hline $6 \mathrm{R}-2,56 \mathrm{~cm}$ (Piece 5) & 56.79 & GN & 6 & vein & C & 2 & 194 & 41 \\
\hline $6 \mathrm{R}-2,69 \mathrm{~cm}$ (Piece 5) & 56.92 & $\mathrm{GN}$ & 6 & vein & C & 3 & 167 & 61 \\
\hline $6 \mathrm{R}-2,67 \mathrm{~cm}$ (Piece 5) & 56.90 & GN & 6 & vein & $\mathrm{S}$ & 4 & 007 & 40 \\
\hline $6 \mathrm{R}-2,66 \mathrm{~cm}$ (Piece 5) & 56.89 & GN & 6 & vein & S & 5 & 165 & 47 \\
\hline $6 \mathrm{R}-2,68 \mathrm{~cm}$ (Piece 5) & 56.91 & GN & 6 & vein & $S$ & 6 & 144 & 30 \\
\hline $6 \mathrm{R}-2,54 \mathrm{~cm}$ (Piece 5) & 56.77 & GN & 6 & vein & C & 7 & 204 & 53 \\
\hline $6 \mathrm{R}-2.50 \mathrm{~cm}$ (Piece 5) & 56.73 & $\mathrm{GN}$ & 6 & vein & $\mathrm{S}$ & 8 & 189 & 46 \\
\hline 6R-2, $83 \mathrm{~cm}$ (Piece 6) & 57.06 & GN & 6 & vein & S & 1 & 208 & 59 \\
\hline 6R-2, $72 \mathrm{~cm}$ (Piece 6) & 56.95 & $\mathrm{GN}$ & 6 & vein & $\mathrm{C}$ & 2 & 084 & 59 \\
\hline $6 \mathrm{R}-2,73 \mathrm{~cm}$ (Piece 6) & 56.96 & GN & 6 & vein & $\mathrm{S}$ & 3 & 125 & 68 \\
\hline $6 \mathrm{R}-2,72 \mathrm{~cm}$ (Piece 6) & 56.95 & GN & 6 & vein & C & 4 & 084 & 62 \\
\hline 6R-2,91 cm (Piece 6) & 57.14 & GN & 6 & vein & $\mathrm{S}$ & 5 & 343 & 70 \\
\hline $6 \mathrm{R}-2,101 \mathrm{~cm}$ (Piece 8) & 57.24 & GN & 6 & vein & S & 1 & 184 & 44 \\
\hline $6 \mathrm{R}-2,100 \mathrm{~cm}$ (Piece 8) & 57.23 & GN & 6 & vein & S & 2 & 171 & 48 \\
\hline 6R-2, $105 \mathrm{~cm}$ (Piece 9) & 57.28 & GN & 6 & vein & $\mathrm{S}$ & 1 & 349 & $7 I$ \\
\hline 6R-2, $111 \mathrm{~cm}$ (Piece 9) & 57.34 & GN & 6 & vein & S & 2 & 354 & 63 \\
\hline $6 \mathrm{R}-2,109 \mathrm{~cm}$ (Piece 9) & 57,32 & GN & 6 & vein & $\mathrm{S}$ & 3 & 035 & 32 \\
\hline 6R-2, $109 \mathrm{~cm}$ (Piece 9) & 57.32 & GN & 6 & vein & S & 4 & 209 & 67 \\
\hline $6 \mathrm{R}-2,122 \mathrm{~cm}$ (Piece 10) & 57.45 & GN & 6 & vein & C & 1 & 189 & 52 \\
\hline $6 \mathrm{R}-2,121 \mathrm{~cm}$ (Piece 10) & 57.44 & GN & 6 & vein & $\mathrm{S}$ & 3 & 318 & 30 \\
\hline $6 \mathrm{R}-3,2 \mathrm{~cm}$ (Piece 1) & 57.75 & GN & 6 & vein & S & 1 & 357 & 64 \\
\hline 7R-1, $45 \mathrm{~cm}$ (Piece 9) & 65.25 & GN & 6 & vein & C & 1 & 348 & 56 \\
\hline $7 \mathrm{R}-1,56 \mathrm{~cm}$ (Piece 11) & 65.36 & GN & 6 & vein & A & 1 & 347 & 59 \\
\hline 7R-1, $94 \mathrm{~cm}$ (Piece 14) & 65.74 & $\mathrm{GN}$ & 6 & vein & $\mathrm{S}$ & 1 & 186 & 71 \\
\hline $7 \mathrm{R}-1,88 \mathrm{~cm}$ (Piece 14) & 65.68 & GN & 6 & vein & $\mathrm{S}$ & 2 & 189 & 75 \\
\hline 7R-1,87 cm (Piece 14) & 65.67 & GN & 6 & vein & $\mathrm{S}$ & 3 & 215 & 51 \\
\hline $7 \mathrm{R}-1.103 \mathrm{~cm}$ (Piece 14) & 65.83 & GN & 6 & vein & $\mathrm{S}$ & 4 & 170 & 56 \\
\hline $7 \mathrm{R}-1,105 \mathrm{~cm}$ (Piece I5) & 65.85 & GN & 6 & fault brecc & & & & \\
\hline $7 \mathrm{R}-1,132 \mathrm{~cm}$ (Piece 19) & 66.12 & GN & 6 & vein & S & 1 & 350 & 67 \\
\hline 7R-1, $132 \mathrm{~cm}$ (Piece 19) & 66.12 & $\mathrm{GN}$ & 6 & vein & S & 2 & 122 & 76 \\
\hline 7R-2, I cm (Piece 1) & 66.23 & GN & 6 & vein & $\mathrm{S}$ & 1 & 171 & 63 \\
\hline $7 \mathrm{R}-2,5 \mathrm{~cm}$ (Piece 1) & 66.27 & GN & 6 & vein & $\mathrm{S}$ & 2 & 170 & 51 \\
\hline $7 \mathrm{R}-2,10 \mathrm{~cm}$ (Piece 1) & 66.32 & GN & 6 & vein & S & 3 & 190 & 73 \\
\hline 7R-2, $8 \mathrm{~cm}$ (Piece 1) & 66.30 & $\mathrm{GN}$ & 6 & foliation & & 4 & 195 & 56 \\
\hline 7R-2, $28 \mathrm{~cm}$ (Piece 3 ) & 66.50 & GN & 6 & vein & S & 1 & 174 & 65 \\
\hline $7 \mathrm{R}-2,32 \mathrm{~cm}$ (Piece 4) & 66.54 & GN & 6 & vein & C & i & 166 & 50 \\
\hline $7 \mathrm{R}-2,36 \mathrm{~cm}$ (Piece 4) & 66.58 & GN & 6 & vein & C & 11 & 189 & 90 \\
\hline $7 \mathrm{R}-2,39 \mathrm{~cm}$ (Piece 4) & 66.61 & GN & 6 & fels dikelet & & 12 & 198 & 69 \\
\hline 7R-2, $54 \mathrm{~cm}$ (Piece 6) & 66.76 & GN & 6 & vein & C & 1 & 150 & 90 \\
\hline 7R-2, $59 \mathrm{~cm}$ (Piece 6) & 66.81 & GN & 6 & vein & C & 2 & 298 & 15 \\
\hline $7 \mathrm{R}-2,56 \mathrm{~cm}$ (Piece 6) & 66.78 & $\mathrm{GN}$ & 6 & vein & $S$ & 3 & 343 & 76 \\
\hline $7 \mathrm{R}-2,53 \mathrm{~cm}$ (Piece 6) & 66.75 & GN & 6 & vein & $\mathrm{S}$ & 4 & 343 & 80 \\
\hline $7 \mathrm{R}-2,60 \mathrm{~cm}$ (Piece 6) & 66.82 & $\mathrm{GN}$ & 6 & vein & $\mathrm{C}$ & 5 & 026 & 57 \\
\hline $7 \mathrm{R}-2,60 \mathrm{~cm}$ (Piece 6) & 66.82 & GN & 6 & vein & C & 6 & 306 & 13 \\
\hline $7 \mathrm{R}-2,70 \mathrm{~cm}$ (Piece 7) & 66.92 & GN & 6 & vein & C & 1 & 342 & 44 \\
\hline $7 \mathrm{R}-2,70 \mathrm{~cm}$ (Piece 7) & 66.92 & GN & 6 & vein & $\mathrm{C}$ & 2 & 357 & 50 \\
\hline $8 \mathrm{R}-1,11 \mathrm{~cm}$ (Piece 2) & 68.61 & GN & 6 & vein & C & 1 & 232 & 66 \\
\hline $8 \mathrm{R}-1,11 \mathrm{~cm}$ (Piece 2) & 68.61 & GN & 6 & vein & C & 2 & 161 & 46 \\
\hline $8 \mathrm{R}-1,13 \mathrm{~cm}$ (Piece 2) & 68.63 & GN & 6 & vein & $\mathrm{C}$ & 3 & 116 & 57 \\
\hline $8 \mathrm{R}-1,14 \mathrm{~cm}$ (Piece 2) & 68.64 & GN & 6 & vein & C & 4 & 281 & 39 \\
\hline $8 \mathrm{R}-1,14 \mathrm{~cm}$ (Piece 2) & 68.64 & GN & 6 & vein & C & 5 & 133 & 75 \\
\hline $8 \mathrm{R}-1,42 \mathrm{~cm}$ (Piece 5) & 68.92 & $\mathrm{GN}$ & 6 & vein & $\mathrm{C}$ & 3 & 350 & 37 \\
\hline $8 \mathrm{R}-1.47 \mathrm{~cm}$ (Piece 5) & 68.97 & GN & 6 & vein & $\mathrm{C}$ & 4 & 022 & 55 \\
\hline $8 \mathrm{R}-1,51 \mathrm{~cm}$ (Piece 5) & 69.01 & GN & 6 & vein & C & 5 & 048 & 49 \\
\hline $8 \mathrm{R}-1,61 \mathrm{~cm}$ (Piece 5) & 69.11 & GN & 6 & vein & C & 6 & 178 & 50 \\
\hline $8 \mathrm{R}-1,70 \mathrm{~cm}$ (Piece 5) & 69.20 & GN & 6 & vein & $\mathbf{Z}$ & 7 & 045 & 59 \\
\hline
\end{tabular}


Table 1 (continued).

\begin{tabular}{|c|c|c|c|c|c|c|c|c|}
\hline & Depth & & & & Vein & & Con & bined \\
\hline Sample number & (mbsf) & Lith. & Unit & Feature type & fill & Feat. \# & $\operatorname{str}\left({ }^{\circ}\right)$ & $\operatorname{dip}\left({ }^{\circ}\right)$ \\
\hline $8 \mathrm{R}-1,74 \mathrm{~cm}$ (Piece 5) & 69.24 & GN & 6 & vein & $\mathrm{Z}$ & 8 & 220 & 62 \\
\hline $8 \mathrm{R}-1,41 \mathrm{~cm}$ (Piece 5 ) & 68.91 & GN & 6 & vein & $\mathrm{C}$ & 9 & 301 & 64 \\
\hline $8 \mathrm{R}-1,71 \mathrm{~cm}$ (Piece 5) & 69.21 & GN & 6 & vein & $\mathrm{Z}$ & 10 & 177 & 60 \\
\hline $8 \mathrm{R}-1,63 \mathrm{~cm}$ (Piece 5) & 69.13 & GN & 6 & vein & $\mathrm{C}$ & 11 & 354 & 86 \\
\hline $8 \mathrm{R}-1,37 \mathrm{~cm}$ (Piece 5) & 68.87 & $\mathrm{GN}$ & 6 & vein & $\mathrm{C}$ & 12 & 240 & 40 \\
\hline $8 \mathrm{R}-1,68 \mathrm{~cm}$ (Piece 5) & 69.18 & GN & 6 & vein & $\mathrm{C}$ & 13 & 179 & 62 \\
\hline $8 \mathrm{R}-1,83 \mathrm{~cm}$ (Piece 5) & 69.33 & $\mathrm{GN}$ & 6 & vein & $\mathrm{C}$ & 14 & 307 & 53 \\
\hline $8 \mathrm{R}-1,82 \mathrm{~cm}$ (Piece 5) & 69.32 & GN & 6 & vein & $\mathrm{C}$ & is & 320 & 43 \\
\hline $8 \mathrm{R}-1,116 \mathrm{~cm}$ (Piece 10) & 69.66 & $\mathrm{GN}$ & 6 & vein & $\mathrm{C}$ & 1 & 205 & 59 \\
\hline $8 \mathrm{R}-1,120 \mathrm{~cm}$ (Piece 10) & 69.70 & $\mathrm{GN}$ & 6 & vein & $\mathrm{C}$ & 2 & 188 & 69 \\
\hline $8 \mathrm{R}-1,137 \mathrm{~cm}$ (Piece 10) & 69.87 & GN & 6 & vein & $\mathrm{C}$ & 3 & 160 & 56 \\
\hline $8 \mathrm{R}-1,140 \mathrm{~cm}$ (Piece 11) & 69.90 & $\mathrm{GN}$ & 6 & vein & $\mathrm{C}$ & 1 & 121 & 59 \\
\hline $8 \mathrm{R}-1,140 \mathrm{~cm}$ (Piece 11) & 69.90 & GN & 6 & vein & $\mathrm{C}$ & 2 & 168 & 59 \\
\hline $8 \mathrm{R}-1,145 \mathrm{~cm}$ (Piece 11) & 69.95 & $\mathrm{GN}$ & 6 & vein & $\mathrm{C}$ & 3 & 118 & 75 \\
\hline $8 \mathrm{R}-1,149 \mathrm{~cm}$ (Piece 11) & 69.99 & GN & 6 & vein & $\mathrm{C}$ & 4 & 191 & 55 \\
\hline $8 \mathrm{R}-2,1 \mathrm{~cm}$ (Piece 1) & 70.02 & GN & 6 & vein & $\mathrm{C}$ & 1 & 357 & 80 \\
\hline $8 \mathrm{R}-2,3 \mathrm{~cm}$ (Piece 1) & 70.04 & GN & 6 & vein & $\mathrm{C}$ & 2 & 169 & 59 \\
\hline $8 \mathrm{R}-2,6 \mathrm{~cm}$ (Piece 1) & 70.07 & GN & 6 & vein & $\mathrm{C}$ & 3 & 192 & 60 \\
\hline $8 \mathrm{R}-2,13 \mathrm{~cm}$ (Piece 1) & 70.14 & GN & 6 & vein & $\mathrm{C}$ & 4 & 158 & 53 \\
\hline $8 \mathrm{R}-2,2 \mathrm{~cm}$ (Piece 1) & 70.03 & GN & 6 & vein & $\mathrm{C}$ & 5 & 232 & 71 \\
\hline $8 \mathrm{R}-2,32 \mathrm{~cm}$ (Piece 4) & 70.33 & GN & 6 & vein & $\mathrm{S}$ & 1 & 354 & 52 \\
\hline $8 \mathrm{R}-2,31 \mathrm{~cm}$ (Piece 4) & 70.32 & GN & 6 & vein & S & 2 & 176 & 90 \\
\hline $8 \mathrm{R}-2,31 \mathrm{~cm}($ Piece 4$)$ & 70.32 & GN & 6 & vein & S & 3 & 187 & 90 \\
\hline $8 \mathrm{R}-2,33 \mathrm{~cm}$ (Piece 4) & 70.34 & GN & 6 & foliation & & 4 & 240 & 80 \\
\hline $8 \mathrm{R}-2,87 \mathrm{~cm}$ (Piece 8 ) & 70.88 & GN & 6 & vein & S & 1 & 182 & 90 \\
\hline $8 \mathrm{R}-2,87 \mathrm{~cm}$ (Piece 8) & 70.88 & GN & 6 & foliation & & 2 & 075 & 83 \\
\hline $8 \mathrm{R}-2,107 \mathrm{~cm}$ (Piece 10) & 71.08 & GN & 6 & vein & S & $i$ & 193 & 79 \\
\hline $9 \mathrm{R}-1,8 \mathrm{~cm}$ (Piece 2$)$ & 70.09 & GN & 6 & vein & S & i & 170 & 68 \\
\hline $9 \mathrm{R}-1,13 \mathrm{~cm}$ (Piece 2) & 70.14 & GN & 6 & vein & $\mathrm{A}$ & 2 & 183 & 61 \\
\hline $9 \mathrm{R}-1,33 \mathrm{~cm}$ (Piece 6) & 74.73 & GN & 6 & vein & $\mathrm{S}$ & 1 & 168 & 38 \\
\hline $9 \mathrm{R}-1,35 \mathrm{~cm}$ (Piece 6) & 74.75 & GN & 6 & vein & s & 2 & 155 & 16 \\
\hline 9R-1, $48 \mathrm{~cm}$ (Piece 7) & 74.88 & GN & 6 & vein & $\mathrm{S}$ & 1 & 176 & 42 \\
\hline $9 \mathrm{R}-1,79 \mathrm{~cm}$ (Piece 11) & 75.19 & GN & 6 & vein & S & i & 307 & 59 \\
\hline $9 \mathrm{R}-1,86 \mathrm{~cm}$ (Piece 11) & 75.26 & GN & 6 & vein & S & 2 & 040 & 58 \\
\hline $9 \mathrm{R}-1,99 \mathrm{~cm}$ (Piece 15) & 75.39 & GN & 6 & vein & A & 1 & 345 & 47 \\
\hline 9R-1, $102 \mathrm{~cm}$ (Piece 15) & 75.42 & GN & 6 & foliation & & 2 & 240 & 63 \\
\hline $9 \mathrm{R}-1,99 \mathrm{~cm}$ (Piece 15) & 75.39 & GN & 6 & ign contact & & 3 & 190 & 15 \\
\hline $9 \mathrm{R}-1,113 \mathrm{~cm}$ (Piece 16) & 75.53 & GN & 6 & vein & $\mathrm{C}$ & 1 & 170 & 72 \\
\hline $9 \mathrm{R}-1,116 \mathrm{~cm}$ (Piece 16) & 75.56 & GN & 6 & vein & S & 2 & 177 & 69 \\
\hline $9 \mathrm{R}-1,127 \mathrm{~cm}$ (Piece 16) & 75.67 & GN & 6 & vein & S & 3 & 183 & 40 \\
\hline $9 \mathrm{R}-2,9 \mathrm{~cm}$ (Piece I) & 75.81 & GN & 6 & yein & $\mathrm{C}$ & 1 & 115 & 74 \\
\hline 9R-2, $4 \mathrm{~cm}$ (Piece 1) & 75.76 & GN & 6 & vein & $\mathrm{s}$ & 2 & 170 & 73 \\
\hline 9R-2, $20 \mathrm{~cm}$ (Piece 1) & 75.92 & GN & 6 & vein & $\mathrm{C}$ & 3 & 171 & 62 \\
\hline $9 \mathrm{R}-2,26 \mathrm{~cm}$ (Piece 1) & 75.98 & GN & 6 & vein & $\mathrm{C}$ & 4 & 157 & 58 \\
\hline $9 \mathrm{R}-2,29 \mathrm{~cm}$ (Piece 1) & 76.01 & GN & 6 & vein & $\mathrm{C}$ & 5 & 146 & 64 \\
\hline $9 \mathrm{R}-2,32 \mathrm{~cm}$ (Piece 1) & 76.04 & GN & 6 & vein & $\mathrm{C}$ & 6 & 178 & 48 \\
\hline $9 \mathrm{R}-2,13 \mathrm{~cm}$ (Piece 1) & 75.85 & GN & 6 & vein & $\mathrm{C}$ & 7 & 197 & 51 \\
\hline $9 \mathrm{R}-2,2 \mathrm{~cm}$ (Piece 1) & 75.74 & GN & 6 & vein & $\mathrm{C}$ & 8 & 101 & 78 \\
\hline 9R-2, $35 \mathrm{~cm}$ (Piece 2) & 76.07 & GN & 6 & vein & $\mathrm{C}$ & 1 & 182 & 59 \\
\hline 9R-2, $38 \mathrm{~cm}$ (Piece 2) & 76.10 & GN & 6 & vein & $\mathrm{C}$ & 2 & 122 & 73 \\
\hline $9 \mathrm{R}-2,40 \mathrm{~cm}$ (Piece 2) & 76.12 & $\mathrm{GN}$ & 6 & vein & $\mathrm{C}$ & 3 & 185 & 41 \\
\hline $9 \mathrm{R}-2,45 \mathrm{~cm}$ (Piece 2 ) & 76.17 & GN & 6 & vein & $\mathrm{C}$ & 4 & 180 & 63 \\
\hline $9 \mathrm{R}-2,49 \mathrm{~cm}$ (Piece 2) & 76.21 & GN & 6 & vein & $\mathrm{C}$ & 6 & 165 & 36 \\
\hline $9 \mathrm{R}-2,51 \mathrm{~cm}$ (Piece 2) & 76.23 & GN & 6 & vein & $\mathrm{C}$ & 7 & 172 & 89 \\
\hline $9 \mathrm{R}-2,74 \mathrm{~cm}$ (Piece 4) & 76.46 & $\mathrm{GN}$ & 6 & vein & $\mathrm{C}$ & 4 & 186 & 63 \\
\hline $9 \mathrm{R}-2,76 \mathrm{~cm}$ (Piece 4) & 76.48 & GN & 6 & vein & $\mathrm{S}$ & 5 & 183 & 71 \\
\hline $9 \mathrm{R}-2,88 \mathrm{~cm}$ (Piece 5) & 76.60 & GN & 6 & vein & S & 1 & 183 & 48 \\
\hline $9 \mathrm{R}-2,96 \mathrm{~cm}$ (Piece 5) & 76.68 & GN & 6 & vein & S & 3 & 190 & 52 \\
\hline $9 \mathrm{R}-2,106 \mathrm{~cm}$ (Piece 5) & 76.78 & GN & 6 & vein & S & 4 & 151 & 55 \\
\hline $9 \mathrm{R}-2,118 \mathrm{~cm}$ (Piece 5) & 76.90 & GN & 6 & vein & S & 5 & 150 & 74 \\
\hline $9 \mathrm{R}-2,78 \mathrm{~cm}$ (Piece 5) & 76.50 & GN & 6 & vein & s & 6 & 180 & 37 \\
\hline $9 \mathrm{R}-2,80 \mathrm{~cm}$ (Piece 5) & 76.52 & GN & 6 & vein & s & 7 & 325 & 38 \\
\hline $9 \mathrm{R}-2,89 \mathrm{~cm}$ (Piece 5) & 76.61 & GN & 6 & vein & S & 9 & 172 & 50 \\
\hline $9 \mathrm{R}-2,95 \mathrm{~cm}$ (Piece 5) & 76.67 & $\mathrm{GN}$ & 6 & vein & S & 10 & 029 & 16 \\
\hline $9 \mathrm{R}-2,105 \mathrm{~cm}$ (Piece 5) & 76.77 & GN & 6 & vein & s & 11 & 028 & 51 \\
\hline $9 \mathrm{R}-3,7 \mathrm{~cm}$ (Piece 1) & 77.05 & GN & 6 & vein & S & 1 & 166 & 50 \\
\hline $9 \mathrm{R}-3,11 \mathrm{~cm}$ (Piece 1) & 77.09 & $\mathrm{GN}$ & 6 & vein & $S$ & 2 & 180 & 60 \\
\hline $9 \mathrm{R}-3,19 \mathrm{~cm}$ (Piece 1) & 77.17 & GN & 6 & vein & $\mathrm{S}$ & 4 & 142 & 53 \\
\hline $9 \mathrm{R}-3,16 \mathrm{~cm}$ (Piece 1) & 77.14 & $\mathrm{GN}$ & 6 & vein & S & 5 & 016 & 49 \\
\hline 9R-3,22 cm (Piece 1) & 77.20 & $\mathrm{GN}$ & 6 & vein & $\mathrm{s}$ & 6 & 173 & 47 \\
\hline 9R-3,29 cm (Piece 1) & 77.27 & GN & 6 & vein & S & 9 & 015 & 75 \\
\hline $9 \mathrm{R}-3,30 \mathrm{~cm}$ (Piece 1) & 77.28 & $\mathrm{GN}$ & 6 & vein & $S$ & 10 & 169 & 55 \\
\hline $9 \mathrm{R}-3,33 \mathrm{~cm}$ (Piece 1) & 77.31 & $\mathrm{GN}$ & 6 & vein & $\mathrm{S}$ & 11 & 191 & 37 \\
\hline 9R-3, $51 \mathrm{~cm}$ (Piece 4) & 77.49 & $\mathrm{GN}$ & 6 & vein & A & 1 & 247 & 74 \\
\hline 9R-3,64 cm (Piece 5) & 77.62 & GN & 6 & vein & $\mathrm{S}$ & 2 & 217 & 26 \\
\hline $9 \mathrm{R}-3,72 \mathrm{~cm}$ (Piece 5) & 77.70 & $\mathrm{GN}$ & 6 & vein & $\mathrm{S}$ & 3 & 020 & 56 \\
\hline $9 \mathrm{R}-3,84 \mathrm{~cm}$ (Piece 5) & 77.82 & $\mathrm{GN}$ & 6 & vein & S & 4 & 254 & 40 \\
\hline $9 \mathrm{R}-3,85 \mathrm{~cm}$ (Piece 5 ) & 77.83 & GN & 6 & vein & S & 5 & 280 & 77 \\
\hline $9 \mathrm{R}-3,91 \mathrm{~cm}$ (Piece 5) & 77.89 & GN & 6 & vein & $S$ & 7 & 017 & 75 \\
\hline $9 \mathrm{R}-3,94 \mathrm{~cm}$ (Piece 5) & 77.92 & GN & 6 & vein & $S$ & 10 & 345 & 74 \\
\hline 9R-3,96 cm (Piece 5) & 77.94 & $\mathrm{GN}$ & 6 & vein & $\mathrm{S}$ & 8 & 334 & 54 \\
\hline 9R-3,99 cm (Piece 5) & 77.97 & OGN & 7 & vein & $\mathrm{S}$ & 9 & 260 & 81 \\
\hline $9 \mathrm{R}-3,102 \mathrm{~cm}$ (Piece 6) & 78.00 & OGN & 7 & vein & $\mathrm{S}$ & 2 & 160 & 87 \\
\hline 9R-3, $104 \mathrm{~cm}$ (Piece 6) & 78.02 & OGN & 7 & vein & $\mathrm{S}$ & 1 & 015 & 53 \\
\hline $9 \mathrm{R}-3,103 \mathrm{~cm}$ (Piece 6) & 78.01 & OGN & 7 & vein & S & 3 & 000 & 63 \\
\hline 9R-3, $129 \mathrm{~cm}$ (Piece 10) & 78.27 & OGN & 7 & vein & $\mathrm{S}$ & 1 & 169 & 48 \\
\hline $9 \mathrm{R}-3,130 \mathrm{~cm}$ (Piece 10$)$ & 78.28 & OGN & 7 & vein & $\mathrm{S}$ & 2 & 191 & 48 \\
\hline $9 \mathrm{R}-3,134 \mathrm{~cm}$ (Piece 11) & 78.32 & OGN & 7 & vein & S & 1 & 158 & $\begin{array}{l}40 \\
62\end{array}$ \\
\hline
\end{tabular}


Table 1 (continued).

\begin{tabular}{|c|c|c|c|c|c|c|c|c|}
\hline & Depth & & & & Vein & & Com & ined \\
\hline Sample number & (mbsf) & Lith. & Unit & Feature type & fill & Feat. \# & $\operatorname{str}\left({ }^{\circ}\right)$ & $\operatorname{dip}\left({ }^{\circ}\right)$ \\
\hline 9R-3, $135 \mathrm{~cm}$ (Piece 11) & 78.33 & OGN & 7 & vein & S & 2 & 152 & 90 \\
\hline $9 \mathrm{R}-4,10 \mathrm{~cm}$ (Piece 2) & 78.51 & OGN & 7 & vein & C & 1 & 336 & 64 \\
\hline $9 \mathrm{R}-4,16 \mathrm{~cm}$ (Piece 2) & 78.57 & OGN & 7 & vein & C & 2 & 345 & 70 \\
\hline $9 \mathrm{R}-4,20 \mathrm{~cm}$ (Piece 2) & 78.61 & $\mathrm{OGN}$ & 7 & vein & $\mathrm{C}$ & 3 & 345 & 70 \\
\hline $9 \mathrm{R}-4,30 \mathrm{~cm}$ (Piece 3 ) & 78.71 & OGN & 7 & vein & C & 1 & 013 & 85 \\
\hline $9 \mathrm{R}-4,58 \mathrm{~cm}$ (Piece 4) & 78.99 & OGN & 7 & vein & $\mathrm{C}$ & i & 190 & 45 \\
\hline $9 \mathrm{R}-4,57 \mathrm{~cm}$ (Piece 4) & 78.98 & OGN & 7 & vein & C & 2 & 025 & 48 \\
\hline $9 \mathrm{R}-4,80 \mathrm{~cm}$ (Piece 6) & 79.21 & $\mathrm{OGN}$ & 7 & vein & $\mathrm{C}$ & i & 009 & 68 \\
\hline 9R-4, $81 \mathrm{~cm}$ (Piece 6) & 79.22 & OGN & 7 & foliation & & 2 & 120 & 74 \\
\hline $9 \mathrm{R}-4.91 \mathrm{~cm}$ (Piece 7) & 79.32 & OGN & 7 & vein & C & 1 & 282 & 61 \\
\hline $9 \mathrm{R}-4,91 \mathrm{~cm}$ (Piece 7) & 79.32 & OGN & 7 & vein & C & 2 & 183 & 67 \\
\hline $9 \mathrm{R}-4,92 \mathrm{~cm}$ (Piece 7) & 79.33 & OGN & 7 & foliation & & 3 & 320 & 78 \\
\hline $9 \mathrm{R}-4,111 \mathrm{~cm}$ (Piece 9) & 79.52 & OGN & 7 & vein & S & 1 & 130 & 40 \\
\hline 9R-4, $109 \mathrm{~cm}$ (Piece 9) & 79.50 & OGN & 7 & vein & A & 2 & 187 & 49 \\
\hline $9 \mathrm{R}-4,128 \mathrm{~cm}$ (Piece 11) & 79.69 & OGN & 7 & foliation & & 2 & 120 & 90 \\
\hline $9 \mathrm{R}-4,131 \mathrm{~cm}$ (Piece 11$)$ & 79.72 & OGN & 7 & vein & A & 1 & 040 & 75 \\
\hline $9 \mathrm{R}-4,140 \mathrm{~cm}$ (Piece 12) & 79.81 & OGN & 7 & vein & A & 1 & 315 & 14 \\
\hline $9 \mathrm{R}-4,138 \mathrm{~cm}$ (Piece 12) & 79.79 & OGN & 7 & vein & A & 2 & 048 & 67 \\
\hline 9R-5, $6 \mathrm{~cm}$ (Piece 1) & 79.94 & OGN & 7 & vein & $\mathrm{A}$ & 1 & 015 & 65 \\
\hline 9R-5, $0 \mathrm{~cm}$ (Piece 1) & 79.88 & OGN & 7 & vein & $\mathrm{C}$ & 2 & 004 & 73 \\
\hline $9 \mathrm{R}-5,15 \mathrm{~cm}$ (Piece 2) & 80.03 & $\mathrm{OGN}$ & 7 & foliation & & 1 & 277 & 74 \\
\hline 9R-5, $26 \mathrm{~cm}$ (Piece 3) & 80.14 & OGN & 7 & foliation & & 1 & 155 & 56 \\
\hline 10R-1, $48 \mathrm{~cm}$ (Piece 1) & 79.58 & $\mathrm{G}$ & 8 & fault brecc & & 1 & & \\
\hline $10 \mathrm{R}-1,46 \mathrm{~cm}$ (Piece 10) & 79.56 & G & 8 & vein & C & 1 & 166 & 67 \\
\hline $10 \mathrm{R}-1,58 \mathrm{~cm}$ (Piece 10) & 79.68 & G & 8 & vein & S & 2 & 183 & 48 \\
\hline 10R-1, $60 \mathrm{~cm}$ (Piece 11) & 79.70 & G & 8 & vein & $\mathrm{S}$ & 1 & 019 & 52 \\
\hline $10 \mathrm{R}-1,56 \mathrm{~cm}$ (Piece 11) & 79.66 & $\mathrm{G}$ & 8 & vein & S & 2 & 200 & 52 \\
\hline 10R-1, $65 \mathrm{~cm}$ (Piece 11) & 79.75 & $\mathrm{G}$ & 8 & vein & S & 3 & 193 & 46 \\
\hline 10R-1, $56 \mathrm{~cm}$ (Piece 11) & 79.66 & G & 8 & vein & S & 4 & 131 & 52 \\
\hline 10R-1, $85 \mathrm{~cm}$ (Piece 11) & 79.95 & G & 8 & foliation & & 5 & 240 & 74 \\
\hline 10R-1, $92 \mathrm{~cm}$ (Piece 13) & 80.02 & G & 8 & foliation & & 6 & 115 & 67 \\
\hline 10R-1, $106 \mathrm{~cm}$ (Piece 13) & 80.16 & G & 8 & foliation & & 7 & 218 & 57 \\
\hline 10R-1, $80 \mathrm{~cm}$ (Piece 13) & 79.90 & G & 8 & vein & S & 1 & 107 & 68 \\
\hline 10R-1, $87 \mathrm{~cm}$ (Piece 13) & 79.97 & G & 8 & vein & $\mathrm{S}$ & 2 & 150 & 45 \\
\hline $10 \mathrm{R}-1,88 \mathrm{~cm}$ (Piece 13$)$ & 79.98 & G & 8 & vein & $\mathrm{s}$ & 4 & 127 & 53 \\
\hline 10R-1, $87 \mathrm{~cm}$ (Piece 13) & 79.97 & G & 8 & vein & S & 5 & 232 & 50 \\
\hline $10 \mathrm{R}-1,110 \mathrm{~cm}$ (Piece 15) & 80.20 & G & 8 & vein & $\mathrm{Z}$ & 1 & 188 & 52 \\
\hline 10R-1, $117 \mathrm{~cm}$ (Piece 17) & 80.27 & G & 8 & vein & S & 1 & 216 & 32 \\
\hline 10R-2, $12 \mathrm{~cm}$ (Piece 2) & 80.60 & G & 8 & foliation & & 3 & 249 & 52 \\
\hline $10 \mathrm{R}-2,10 \mathrm{~cm}$ (Piece 2) & 80.58 & G & 8 & vein & S & 1 & 010 & 45 \\
\hline $10 \mathrm{R}-2,8 \mathrm{~cm}$ (Piece 2$)$ & 80.56 & G & 8 & vein & $\mathrm{s}$ & 2 & 247 & 64 \\
\hline $10 \mathrm{R}-2,9 \mathrm{~cm}$ (Piece 2) & 80.57 & G & 8 & vein & S & 3 & 097 & 46 \\
\hline IIR-1, $35 \mathrm{~cm}$ (Piece 1) & 84.45 & GN & 9 & fault brecc & & 1 & & \\
\hline $11 \mathrm{R}-1.48 \mathrm{~cm}$ (Piece 7) & 84.58 & GN & 9 & fault brecc & & 1 & & \\
\hline $11 \mathrm{R}-1,49 \mathrm{~cm}$ (Piece 10) & 84.59 & GN & 9 & vein & $S$ & i & 356 & 60 \\
\hline $11 \mathrm{R}-1,52 \mathrm{~cm}$ (Piece 10) & 84.62 & $\mathrm{GN}$ & 9 & vein & S & 2 & 358 & 60 \\
\hline $11 \mathrm{R}-1,81 \mathrm{~cm}$ (Piece 12) & 84.91 & $\mathrm{GN}$ & 9 & vein & C & 2 & 012 & 46 \\
\hline $11 \mathrm{R}-1,78 \mathrm{~cm}$ (Piece 12) & 84.88 & $\mathrm{GN}$ & 9 & vein & $\mathrm{C}$ & 1 & 028 & 52 \\
\hline $11 \mathrm{R}-1,79 \mathrm{~cm}$ (Piece 12) & 84.89 & GN & 9 & vein & C & 3 & 030 & 46 \\
\hline $11 \mathrm{R}-1,74 \mathrm{~cm}$ (Piece 12) & 84.84 & $\mathrm{GN}$ & 9 & vein & S & 4 & 333 & 41 \\
\hline $1 \mathrm{IR}-1,94 \mathrm{~cm}$ (Piece 14) & 85.04 & GN & 9 & vein & C & 1 & 347 & 49 \\
\hline 11R-1, $95 \mathrm{~cm}$ (Piece 14) & 85.05 & GN & 9 & vein & C & 2 & 355 & 47 \\
\hline $11 \mathrm{R}-1,100 \mathrm{~cm}$ (Piece 14) & 85.10 & $\mathrm{GN}$ & 9 & vein & $\mathrm{C}$ & 3 & 357 & 32 \\
\hline $11 \mathrm{R}-1,117 \mathrm{~cm}$ (Piece 16) & 85.27 & $\mathrm{GN}$ & 9 & vein & $\mathrm{C}$ & 8 & 164 & 43 \\
\hline $11 \mathrm{R}-1,112 \mathrm{~cm}$ (Piece 16) & 85.22 & GN & 9 & vein & C & 1 & 123 & 50 \\
\hline $11 \mathrm{R}-1,129 \mathrm{~cm}$ (Piece 17) & 85.39 & GN & 9 & vein & C & 7 & 358 & 42 \\
\hline $11 \mathrm{R}-1,121 \mathrm{~cm}$ (Piece 17) & 85.31 & $\mathrm{GN}$ & 9 & vein & C & 1 & 045 & 52 \\
\hline IIR-2, $27 \mathrm{~cm}$ (Piece 5) & 85.84 & GN & 9 & vein & $\mathrm{S}$ & 1 & 016 & 52 \\
\hline $11 \mathrm{R}-2,28 \mathrm{~cm}$ (Piece 5) & 85.85 & GN & 9 & vein & C & 2 & 345 & 61 \\
\hline $11 \mathrm{R}-2,33 \mathrm{~cm}$ (Piece 5) & 85.90 & $\mathrm{GN}$ & 9 & vein & S & 3 & 010 & 47 \\
\hline $11 \mathrm{R}-2,39 \mathrm{~cm}$ (Piece 5) & 85.96 & GN & 9 & vein & C & 4 & 008 & 60 \\
\hline $11 \mathrm{R}-2,41 \mathrm{~cm}$ (Piece 5) & 85.98 & $\mathrm{GN}$ & 9 & vein & S & 5 & 015 & 43 \\
\hline $1 \mathrm{R}-2,35 \mathrm{~cm}$ (Piece 5) & 85.92 & GN & 9 & vein & S & 6 & 020 & 46 \\
\hline $11 \mathrm{R}-2,35 \mathrm{~cm}$ (Piece 5) & 85.92 & $\mathrm{GN}$ & 9 & foliation & & 7 & 320 & 70 \\
\hline $11 \mathrm{R}-2.59 \mathrm{~cm}$ (Piece 7) & 86.16 & $\mathrm{GN}$ & 9 & vein & S & 1 & 178 & 79 \\
\hline $11 \mathrm{R}-2,67 \mathrm{~cm}$ (Piece 8) & 86.24 & $\mathrm{GN}$ & 9 & vein & $\mathrm{S}$ & 1 & 355 & 66 \\
\hline $11 \mathrm{R}-2,67 \mathrm{~cm}$ (Piece 8) & 86.24 & $\mathrm{GN}$ & 9 & vein & $\mathrm{S}$ & 2 & 356 & 64 \\
\hline $11 \mathrm{R}-2,85 \mathrm{~cm}$ (Piece 9) & 86.42 & $\mathrm{GN}$ & 9 & vein & $\mathrm{S}$ & 1 & 021 & 67 \\
\hline 1IR-2, $79 \mathrm{~cm}$ (Piece 9) & 86.36 & GN & 9 & vein & S & 2 & 173 & 49 \\
\hline $11 \mathrm{R}-2,95 \mathrm{~cm}$ (Piece 10) & 86.52 & GN & 9 & vein & S & 1 & 189 & 66 \\
\hline $11 \mathrm{R}-2,92 \mathrm{~cm}$ (Piece 10) & 86.49 & GN & 9 & vein & $\mathrm{S}$ & 2 & 108 & 50 \\
\hline $11 \mathrm{R}-2,95 \mathrm{~cm}$ (Piece 10) & 86.52 & GN & 9 & vein & S & 3 & 161 & 24 \\
\hline $11 \mathrm{R}-2,112 \mathrm{~cm}$ (Piece 11) & 86.69 & $\mathrm{GN}$ & 9 & vein & $\mathrm{S}$ & 1 & 357 & 75 \\
\hline $11 \mathrm{R}-2,115 \mathrm{~cm}$ (Piece 12) & 86.72 & GN & 9 & vein & S & i & 081 & 75 \\
\hline $11 \mathrm{R}-2,129 \mathrm{~cm}$ (Piece 12) & 86.86 & GN & 9 & vein & $\mathrm{S}$ & 2 & 021 & 63 \\
\hline $11 \mathrm{R}-2,120 \mathrm{~cm}$ (Piece 12) & 86.77 & GN & 9 & vein & $\mathrm{Z}$ & 3 & 257 & 38 \\
\hline $11 \mathrm{R}-2,123 \mathrm{~cm}$ (Piece 12) & 86.80 & GN & 9 & foliation & & 4 & 135 & 72 \\
\hline $11 \mathrm{R}-3,0 \mathrm{~cm}$ (Piece 1) & 87.02 & GN & 9 & vein & $\mathrm{S}$ & 1 & 107 & 60 \\
\hline $11 \mathrm{R}-3,11 \mathrm{~cm}$ (Piece 1) & 87.13 & GN & 9 & vein & S & 2 & 178 & 64 \\
\hline $11 \mathrm{R}-3,16 \mathrm{~cm}$ (Piece 3 ) & 87.18 & GN & 9 & vein & $\mathrm{S}$ & 1 & 009 & 72 \\
\hline 1IR-3, $45 \mathrm{~cm}$ (Piece 6) & 87.47 & OGN & 10 & vein & $\mathrm{s}$ & $i$ & 164 & 90 \\
\hline $11 \mathrm{R}-3,47 \mathrm{~cm}$ (Piece 6) & 87.49 & OGN & 10 & vein & A & 2 & 345 & 69 \\
\hline $12 \mathrm{R}-1,8 \mathrm{~cm}$ (Piece 1) & 93.88 & GN & 11 & fault brecc & & 1 & & \\
\hline $12 \mathrm{R}-1,35 \mathrm{~cm}$ (Piece 5) & 94.15 & GN & 11 & fault brecc & & $i$ & & \\
\hline $12 \mathrm{R}-1.80 \mathrm{~cm}$ (Piece 10) & 94.60 & GN & 11 & fault brecc & & i & & \\
\hline 12R-1, $92 \mathrm{~cm}$ (Piece II) & 94.72 & GN & 11 & fault brecc & & 1 & & \\
\hline $12 \mathrm{R}-1,97 \mathrm{~cm}$ (Piece 12) & 94.77 & GN & 11 & vein & C & 1 & 172 & 60 \\
\hline 12R-1, $104 \mathrm{~cm}$ (Piece 12) & 94.84 & GN & 11 & vein & $\mathrm{S}$ & 2 & 123 & 18 \\
\hline
\end{tabular}


Table 1 (continued).

\begin{tabular}{|c|c|c|c|c|c|c|c|c|}
\hline & Depth & & & & Vein & & Con & pined \\
\hline Sample number & (mbsf) & Lith. & Unit & Feature type & fill & Feat. \# & $\operatorname{str}\left({ }^{\circ}\right)$ & $\operatorname{dip}\left({ }^{\circ}\right)$ \\
\hline $12 \mathrm{R}-1,109 \mathrm{~cm}$ (Piece 12) & 94.89 & GN & 11 & vein & $S$ & 3 & 198 & 46 \\
\hline $12 \mathrm{R}-1,115 \mathrm{~cm}$ (Piece 12$)$ & 94.95 & GN & 11 & vein & $\mathrm{S}$ & 4 & 194 & 41 \\
\hline $12 \mathrm{R}-1,121 \mathrm{~cm}$ (Piece 12) & 95.01 & GN & 11 & vein & $\mathrm{C}$ & 5 & 195 & 51 \\
\hline $12 \mathrm{R}-1,101 \mathrm{~cm}$ (Piece 12) & 94.81 & GN & 11 & vein & $\mathrm{S}$ & 6 & 223 & 58 \\
\hline $12 \mathrm{R}-1,103 \mathrm{~cm}$ (Piece 12) & 94.83 & GN & 11 & vein & $\mathrm{s}$ & 7 & 220 & 52 \\
\hline $12 \mathrm{R}-1,110 \mathrm{~cm}$ (Piece 12) & 94.90 & GN & 11 & vein & $\mathrm{C}$ & 8 & 195 & 42 \\
\hline 12R-1, $128 \mathrm{~cm}$ (Piece 13) & 95.08 & GN & 11 & fault brecc & & 1 & & \\
\hline 12R-2, $53 \mathrm{~cm}$ (Piece 4) & 95.85 & GN & 11 & vein & C & i & 182 & 44 \\
\hline $12 \mathrm{R}-2,58 \mathrm{~cm}$ (Piece 4) & 95.90 & GN & 11 & vein & $\mathrm{S}$ & 2 & 205 & 56 \\
\hline $12 \mathrm{R}-2,21 \mathrm{~cm}$ (Piece 4) & 95.53 & GN & II & vein & $\mathrm{C}$ & 3 & 138 & 43 \\
\hline $12 \mathrm{R}-2,35 \mathrm{~cm}$ (Piece 4) & 95.67 & $\mathrm{GN}$ & 11 & vein & $\mathrm{S}$ & 4 & 119 & 55 \\
\hline 12R-2, $34 \mathrm{~cm}$ (Piece 4) & 95.66 & GN & 11 & vein & $\mathrm{S}$ & 5 & 245 & 30 \\
\hline 12R-2, $33 \mathrm{~cm}$ (Piece 4) & 95.65 & GN & 11 & vein & $\mathrm{S}$ & 6 & 139 & 35 \\
\hline $12 \mathrm{R}-2,42 \mathrm{~cm}$ (Piece 4) & 95.74 & GN & 11 & foliation & & 10 & 045 & 90 \\
\hline $12 \mathrm{R}-2,62 \mathrm{~cm}$ (Piece 4) & 95.94 & GN & 11 & vein & $\mathrm{S}$ & 8 & 258 & 61 \\
\hline 12R-2, $64 \mathrm{~cm}$ (Piece 4) & 95.96 & GN & 11 & vein & $\mathrm{S}$ & 9 & 243 & 44 \\
\hline $12 \mathrm{R}-2,70 \mathrm{~cm}$ (Piece 5) & 96.02 & GN & 11 & fault brecc & & 1 & & \\
\hline $12 \mathrm{R}-2,87 \mathrm{~cm}$ (Piece 9) & 96.19 & GN & 11 & vein & $\mathrm{S}$ & i & 135 & 55 \\
\hline 12R-2, $98 \mathrm{~cm}$ (Piece 9) & 96.30 & GN & 11 & vein & $\mathrm{S}$ & 2 & 165 & 41 \\
\hline $12 \mathrm{R}-2,90 \mathrm{~cm}$ (Piece 9) & 96.22 & GN & 11 & vein & $\mathrm{S}$ & 3 & 245 & 90 \\
\hline 12R-2, $96 \mathrm{~cm}$ (Piece 9) & 96.28 & GN & 11 & vein & $\mathrm{S}$ & 4 & 190 & 79 \\
\hline 12R-2, $98 \mathrm{~cm}$ (Piece 9) & 96.30 & GN & 11 & ign contact & & 5 & 180 & 85 \\
\hline 12R-2, $98 \mathrm{~cm}$ (Piece 9) & 96.30 & GN & 11 & foliation & & 6 & 165 & 61 \\
\hline 12R-2, $109 \mathrm{~cm}$ (Piece 10) & 96.41 & GN & 11 & vein & $\mathrm{C}$ & 1 & 068 & 44 \\
\hline 12R-2, $129 \mathrm{~cm}$ (Piece 10) & 96.61 & GN & 11 & vein & $\mathrm{S}$ & 2 & 070 & 42 \\
\hline $12 \mathrm{R}-2,120 \mathrm{~cm}$ (Piece 10$)$ & 96.52 & GN & 11 & vein & C & 3 & 162 & 41 \\
\hline 12R-2, $108 \mathrm{~cm}$ (Piece 10) & 96.40 & GN & 11 & vein & $\mathrm{S}$ & 4 & 125 & 82 \\
\hline $12 \mathrm{R}-2,129 \mathrm{~cm}$ (Piece 10) & 96.61 & GN & 11 & vein & $\mathrm{S}$ & 5 & 180 & 70 \\
\hline $12 \mathrm{R}-2,136 \mathrm{~cm}$ (Piece 10$)$ & 96.68 & GN & 11 & foliation & & 6 & 180 & so \\
\hline $12 \mathrm{R}-2,134 \mathrm{~cm}$ (Piece 11) & 96.66 & GN & 11 & vein & C & 1 & 005 & 49 \\
\hline $12 \mathrm{R}-2,140 \mathrm{~cm}$ (Piece 11$)$ & 96.72 & GN & 11 & foliation & & 2 & 030 & 56 \\
\hline $12 \mathrm{R}-3,8 \mathrm{~cm}$ (Piece 1$)$ & 96.84 & GN & 11 & vein & $\mathrm{S}$ & 1 & 059 & 85 \\
\hline $12 \mathrm{R}-3,18 \mathrm{~cm}$ (Piece 1) & 96.94 & GN & 11 & vein & $\mathrm{C}$ & 2 & 352 & 63 \\
\hline $12 \mathrm{R}-3,10 \mathrm{~cm}$ (Piece 1) & 96.86 & GN & 11 & vein & $\mathrm{S}$ & 3 & 075 & 56 \\
\hline 12R-3, $19 \mathrm{~cm}$ (Piece 1) & 96.95 & GN & 11 & vein & $\mathrm{S}$ & 4 & 077 & 50 \\
\hline $12 \mathrm{R}-3,26 \mathrm{~cm}$ (Piece 1) & 97.02 & GN & 11 & vein & $\mathrm{S}$ & 5 & 036 & 39 \\
\hline $12 \mathrm{R}-3,30 \mathrm{~cm}$ (Piece 1) & 97.06 & GN & 11 & vein & $\mathrm{s}$ & 6 & 335 & 80 \\
\hline $12 \mathrm{R}-3,32 \mathrm{~cm}$ (Piece 1) & 97.08 & GN & ii & vein & $\mathrm{S}$ & 7 & 025 & 32 \\
\hline $12 \mathrm{R}-3,34 \mathrm{~cm}$ (Piece 1) & 97.10 & GN & 11 & vein & $\mathrm{S}$ & 8 & 099 & 44 \\
\hline $12 \mathrm{R}-3,39 \mathrm{~cm}$ (Piece 1) & 97.15 & GN & 11 & vein & $\mathrm{S}$ & 9 & 104 & 24 \\
\hline $12 \mathrm{R}-3,45 \mathrm{~cm}$ (Piece 1) & 97.21 & GN & 11 & vein & $\mathrm{S}$ & 10 & 009 & 83 \\
\hline $12 \mathrm{R}-3,60 \mathrm{~cm}$ (Piece 2) & 97.36 & GN & 11 & vein & $\mathrm{S}$ & 1 & 006 & 65 \\
\hline $12 \mathrm{R}-3,50 \mathrm{~cm}$ (Piece 2) & 97.26 & GN & 11 & vein & $\mathrm{S}$ & 2 & 297 & 43 \\
\hline $12 \mathrm{R}-3,54 \mathrm{~cm}$ (Piece 2) & 97.30 & GN & 11 & vein & $\mathrm{S}$ & 3 & 350 & 30 \\
\hline $12 \mathrm{R}-3,57 \mathrm{~cm}$ (Piece 2) & 97.33 & GN & 11 & foliation & & 4 & 284 & 80 \\
\hline $12 \mathrm{R}-3,60 \mathrm{~cm}$ (Piece 3) & 97.36 & GN & 11 & vein & $\mathrm{S}$ & 1 & 348 & 49 \\
\hline $12 \mathrm{R}-3,72 \mathrm{~cm}$ (Piece 3) & 97.48 & GN & 11 & vein & $\mathrm{S}$ & 2 & 040 & 54 \\
\hline $12 \mathrm{R}-3,69 \mathrm{~cm}$ (Piece 3) & 97.45 & GN & 11 & vein & $\mathrm{S}$ & 3 & 007 & 50 \\
\hline $12 \mathrm{R}-3,80 \mathrm{~cm}$ (Piece 4) & 97.56 & GN & 11 & vein & $\mathrm{S}$ & 1 & 359 & 48 \\
\hline 12R-3, $87 \mathrm{~cm}$ (Piece 4) & 97.63 & GN & 11 & vein & $\mathrm{S}$ & 2 & 355 & 55 \\
\hline 12R-3, $94 \mathrm{~cm}$ (Piece 4) & 97.70 & GN & 11 & vein & $\mathrm{s}$ & 3 & 280 & 71 \\
\hline $12 \mathrm{R}-3,84 \mathrm{~cm}$ (Piece 4) & 97.60 & GN & 11 & vein & $\mathrm{S}$ & 4 & 005 & 48 \\
\hline $12 \mathrm{R}-3,103 \mathrm{~cm}$ (Piece 5) & 97.79 & GN & 11 & vein & $\mathrm{C}$ & 1 & 334 & 43 \\
\hline $12 \mathrm{R}-3,114 \mathrm{~cm}$ (Piece 7$)$ & 97.90 & $\mathrm{GN}$ & 11 & vein & C & i & 344 & 41 \\
\hline $12 \mathrm{R}-3,124 \mathrm{~cm}$ (Piece 7) & 98.00 & GN & 11 & vein & C & 2 & 300 & 40 \\
\hline $12 \mathrm{R}-3,141 \mathrm{~cm}$ (Piece 8) & 98.17 & GN & 11 & vein & $\mathrm{C}$ & I & 016 & 46 \\
\hline $12 \mathrm{R}-3,149 \mathrm{~cm}$ (Piece 8 ) & 98.25 & GN & 11 & vein & $\mathrm{S}$ & 2 & 305 & 55 \\
\hline $12 \mathrm{R}-3,147 \mathrm{~cm}$ (Piece 8 ) & 98.23 & GN & 11 & foliation & & 3 & 050 & 70 \\
\hline $12 \mathrm{R}-4,12 \mathrm{~cm}$ (Piece 2) & 98.38 & GN & 11 & vein & $\mathrm{S}$ & 1 & 340 & 17 \\
\hline $12 \mathrm{R}-4,9 \mathrm{~cm}$ (Piece 2) & 98.35 & GN & 11 & vein & $\mathrm{S}$ & 2 & 025 & 77 \\
\hline $12 \mathrm{R}-4,16 \mathrm{~cm}$ (Piece 3 ) & 98.42 & GN & 11 & vein & $\mathrm{S}$ & 1 & 026 & 70 \\
\hline $12 \mathrm{R}-4,40 \mathrm{~cm}$ (Piece 4) & 98.66 & GN & 11 & vein & S & 1 & 182 & 69 \\
\hline $12 \mathrm{R}-4,39 \mathrm{~cm}$ (Piece 4) & 98.65 & GN & 11 & vein & $\mathrm{S}$ & 10 & 270 & 59 \\
\hline $12 \mathrm{R}-4,43 \mathrm{~cm}$ (Piece 4) & 98.69 & GN & 11 & vein & $\mathrm{S}$ & 3 & 186 & 65 \\
\hline $12 \mathrm{R}-4,40 \mathrm{~cm}$ (Piece 4) & 98.66 & GN & 11 & vein & $\mathrm{S}$ & 4 & 186 & 65 \\
\hline $12 \mathrm{R}-4,43 \mathrm{~cm}$ (Piece 4) & 98.69 & GN & 11 & vein & $\mathrm{S}$ & 5 & 186 & 65 \\
\hline $12 \mathrm{R}-4,46 \mathrm{~cm}$ (Piece 4) & 98.72 & GN & 11 & vein & $\mathrm{S}$ & 6 & 186 & 62 \\
\hline $12 \mathrm{R}-4,55 \mathrm{~cm}$ (Piece 4) & 98.81 & GN & 11 & vein & $\mathrm{S}$ & 7 & 140 & 68 \\
\hline $12 \mathrm{R}-4,33 \mathrm{~cm}$ (Piece 4) & 98.59 & GN & 11 & vein & $\mathrm{A}$ & 12 & 044 & 45 \\
\hline $12 \mathrm{R}-4,50 \mathrm{~cm}$ (Piece 4) & 98.76 & GN & 11 & foliation & & 13 & 315 & 68 \\
\hline $12 \mathrm{R}-4,56 \mathrm{~cm}$ (Piece 4) & 98.82 & GN & 11 & vein & $\mathrm{S}$ & 8 & 165 & 63 \\
\hline $12 \mathrm{R}-4,123 \mathrm{~cm}$ (Piece 13) & 99.49 & GN & 11 & vein & $\mathrm{S}$ & 1 & 176 & 65 \\
\hline $12 \mathrm{R}-4,122 \mathrm{~cm}$ (Piece 13) & 99.48 & GN & 11 & vein & $\mathrm{s}$ & 2 & 088 & 51 \\
\hline $12 \mathrm{R}-4,123 \mathrm{~cm}$ (Piece 13) & 99.49 & GN & 11 & foliation & & 3 & 175 & 60 \\
\hline $12 \mathrm{R}-4,134 \mathrm{~cm}$ (Piece 14) & 99.60 & GN & 11 & vein & $\mathrm{S}$ & 1 & 017 & 68 \\
\hline $12 \mathrm{R}-4,131 \mathrm{~cm}$ (Piece 14) & 99.57 & GN & 11 & vein & $\mathrm{S}$ & 4 & 072 & 58 \\
\hline $12 \mathrm{R}-4,140 \mathrm{~cm}$ (Piece 15) & 99.66 & GN & 11 & vein & $\mathrm{S}$ & 1 & 179 & 90 \\
\hline $12 \mathrm{R}-4,140 \mathrm{~cm}$ (Piece 15$)$ & 99.66 & GN & 11 & vein & $\mathrm{S}$ & 2 & 185 & 90 \\
\hline $12 \mathrm{R}-5,35 \mathrm{~cm}$ (Piece 7) & 100.12 & GN & 11 & vein & $\mathrm{S}$ & 1 & 090 & 31 \\
\hline 12R-5, $44 \mathrm{~cm}$ (Piece 7) & 100.21 & GN & 11 & vein & C & 2 & 180 & 50 \\
\hline $12 \mathrm{R}-5,45 \mathrm{~cm}$ (Piece 7) & 100.22 & GN & 11 & vein & C & 3 & 180 & $5 I$ \\
\hline $12 \mathrm{R}-5,49 \mathrm{~cm}$ (Piece 7) & 100.26 & GN & 11 & vein & $\mathrm{C}$ & 4 & 200 & 66 \\
\hline 12R-5, $53 \mathrm{~cm}$ (Piece 7) & 100.30 & GN & 11 & vein & C & 5 & 200 & 66 \\
\hline 12R-5, $54 \mathrm{~cm}$ (Piece 7) & 100.31 & GN & 11 & vein & $\mathrm{S}$ & 6 & 090 & 40 \\
\hline $12 \mathrm{R}-5,60 \mathrm{~cm}$ (Piece 7) & 100.37 & GN & 11 & vein & $\mathrm{S}$ & 7 & 180 & 81 \\
\hline 12R-5, $56 \mathrm{~cm}$ (Piece 7) & 100.33 & GN & 11 & vein & C & 8 & 037 & 28 \\
\hline $12 \mathrm{R}-5,55 \mathrm{~cm}$ (Piece 7) & 100.32 & GN & 11 & foliation & & 9 & 020 & 81 \\
\hline 12R-5, $88 \mathrm{~cm}$ (Piece 9) & 100.65 & GN & 11 & foliation & & 4 & 140 & 90 \\
\hline
\end{tabular}


Table 1 (continued).

\begin{tabular}{|c|c|c|c|c|c|c|c|c|c|c|c|c|c|c|c|}
\hline \multirow[b]{2}{*}{ Sample number } & \multirow{2}{*}{$\begin{array}{l}\text { Depth } \\
\text { (mbsf) }\end{array}$} & \multirow[b]{2}{*}{ Lith. } & \multirow[b]{2}{*}{ Unit } & \multirow[b]{2}{*}{ Feature type } & \multirow{2}{*}{$\begin{array}{l}\text { Vein } \\
\text { fill }\end{array}$} & \multirow[b]{2}{*}{ Feat. \# } & \multicolumn{2}{|c|}{ Combined } & \multicolumn{2}{|c|}{ Stable mag. rem. } & \multirow{2}{*}{$\begin{array}{c}\text { Meas. } \\
\text { by }\end{array}$} & \multicolumn{2}{|c|}{ Rest (D only) } & \multicolumn{2}{|c|}{ Rest (D+I) } \\
\hline & & & & & & & $\operatorname{str}\left({ }^{\circ}\right)$ & $\operatorname{dip}\left({ }^{\circ}\right)$ & $\operatorname{dec}\left({ }^{\circ}\right)$ & $\operatorname{inc}\left({ }^{\circ}\right)$ & & $\operatorname{str}\left({ }^{\circ}\right)$ & $\operatorname{dip}\left({ }^{\circ}\right)$ & $\operatorname{str}\left({ }^{\circ}\right)$ & $\operatorname{dip}\left({ }^{\circ}\right)$ \\
\hline 12R-5, $88 \mathrm{~cm}$ (Piece 9) & 100.65 & GN & 11 & vein & S & 1 & 170 & 45 & -012.0 & 47.1 & $\mathrm{~s}$ & 182 & 45 & 147.0 & 57.5 \\
\hline 12R-5, $87 \mathrm{~cm}$ (Piece 9) & 100.64 & GN & 11 & vein & $\mathrm{s}$ & 2 & 250 & 87 & -012.0 & 47.1 & $\mathrm{~s}$ & 262 & 87 & 258.7 & 45.0 \\
\hline 12R-5, $76 \mathrm{~cm}$ (Piece 9) & 100.53 & GN & 11 & vein & S & 3 & 021 & 21 & -012.0 & 47.1 & $\mathrm{~S}$ & 033 & 21 & 068.8 & 56.2 \\
\hline 12R-5, $97 \mathrm{~cm}$ (Piece 11) & 100.74 & GN & 11 & vein & S & 1 & 359 & 66 & & & & & & & \\
\hline $12 \mathrm{R}-5,99 \mathrm{~cm}$ (Piece 11$)$ & 100.76 & GN & 11 & vein & $\mathrm{S}$ & 2 & 000 & 66 & & & & & & & \\
\hline $12 \mathrm{R}-5,105 \mathrm{~cm}$ (Piece 11 ) & 100.82 & $\mathrm{GN}$ & 11 & vein & S & 3 & 000 & 58 & & & & & & & \\
\hline $12 \mathrm{R}-5,110 \mathrm{~cm}$ (Piece 11$)$ & 100.87 & GN & ii & vein & S & 4 & 015 & 57 & & & & & & & \\
\hline $12 \mathrm{R}-5,111 \mathrm{~cm}$ (Piece 11 ) & 100.88 & GN & 11 & vein & $\mathrm{s}$ & 5 & 015 & 57 & & & & & & & \\
\hline $12 \mathrm{R}-5,112 \mathrm{~cm}$ (Piece 11 ) & 100.89 & GN & 11 & vein & S & 6 & 015 & 57 & & & & & & & \\
\hline $12 \mathrm{R}-5,110 \mathrm{~cm}$ (Piece 11$)$ & 100.87 & GN & 11 & foliation & & 7 & 230 & 73 & & & & & & & \\
\hline 12R-6, $4 \mathrm{~cm}$ (Piece 1) & 101.25 & GN & 11 & foliation & & 1 & 050 & 70 & & & & & & & \\
\hline 12R-6. $32 \mathrm{~cm}$ (Piece 5) & 101.53 & GN & ii & vein & $\mathrm{s}$ & 1 & 173 & 67 & 034.0 & 33 & $\mathrm{C}(+\mathrm{J})$ & 139 & 67 & 134.1 & 86.8 \\
\hline 12R-6, $33 \mathrm{~cm}$ (Piece 5) & 101.54 & GN & 11 & vein & S & 2 & 173 & 67 & 034.0 & 33 & $\mathrm{C}(+\mathrm{J})$ & 139 & 67 & 134.1 & 86.8 \\
\hline $12 \mathrm{R}-6,30 \mathrm{~cm}$ (Piece 5) & 101.51 & GN & ii & vein & $\mathrm{S}$ & 6 & 264 & 90 & 034.0 & 33 & $\mathrm{C}(+\mathrm{J})$ & 230 & 90 & 226.4 & 68.6 \\
\hline 12R-6, $38 \mathrm{~cm}$ (Piece 5) & 101.59 & GN & 11 & vein & $\mathrm{S}$ & 3 & 141 & 73 & 034.0 & 33 & $C(+J)$ & 107 & 73 & 286.5 & 79.8 \\
\hline 12R-6, $37 \mathrm{~cm}$ (Piece 5) & 101.58 & GN & 11 & vein & S & 4 & 143 & 73 & 034.0 & 33 & $\mathrm{C}(+\mathrm{J})$ & 109 & 73 & 288.4 & 80.0 \\
\hline $12 \mathrm{R}-6,41 \mathrm{~cm}$ (Piece 5) & 101.62 & GN & 11 & vein & $\mathrm{S}$ & 5 & 200 & 71 & 034.0 & 33 & $\mathrm{C}(+\mathrm{J})$ & 166 & 71 & 158.8 & 79.8 \\
\hline 12R-6, $57 \mathrm{~cm}$ (Piece 6) & 101.78 & GN & 11 & vein & S & 1 & 180 & 26 & & & & & & & \\
\hline 13R-1, $45 \mathrm{~cm}$ (Piece 7) & 103.85 & GN & 11 & vein & $\mathrm{S}$ & 1 & 180 & 90 & & & & & & & \\
\hline 13R-1, $64 \mathrm{~cm}$ (Piece 9) & 104.04 & GN & 11 & foliation & & 5 & 350 & 80 & 341.0 & 39 & $\mathrm{C}(1 / 2)$ & 009 & 80 & 013.0 & 86.8 \\
\hline 13R-1, $68 \mathrm{~cm}$ (Piece 9) & 104.08 & GN & 11 & vein & S & 2 & 185 & 50 & 341.0 & 39 & $\mathrm{C}(1 / 2)$ & 204 & 50 & 171.4 & 45.1 \\
\hline 13R-1, $60 \mathrm{~cm}$ (Piece 9) & 104.00 & GN & 11 & vein & S & 1 & 327 & 65 & 341.0 & 39 & $C(1 / 2)$ & 346 & 65 & 003.8 & 61.8 \\
\hline $13 \mathrm{R}-1,70 \mathrm{~cm}$ (Piece 9) & 104.10 & $\mathrm{GN}$ & 11 & vein & S & 3 & 358 & 42 & 341.0 & 39 & $\mathrm{C}(1 / 2)$ & 017 & 42 & 042.3 & 59.8 \\
\hline 13R-1, $68 \mathrm{~cm}$ (Piece 9) & 104.08 & GN & 11 & vein & $\mathrm{S}$ & 4 & 167 & 71 & 341.0 & 39 & $\mathrm{C}(1 / 2)$ & 186 & 71 & 173.8 & 71.1 \\
\hline
\end{tabular}

13R-1,77 cm (Piece 11) 104.17 GN 11 vein

13R-1,92 cm (Piece 11) 104.32 GN 11 vein

13R-1, $92 \mathrm{~cm}$ (Piece 11) 104.32 GN 11 foliation

13R-1, $76 \mathrm{~cm}$ (Piece 11) $104.16 \quad \mathrm{GN} \quad 11$ vein

13R-1, 104 cm (Piece 12) 104.41 GN 11 vein

$13 R-1,104 \mathrm{~cm}$ (Piece 12) 104.44 GN 11 vein

$13 R-1,108 \mathrm{~cm}$ (Piece 12) 104.48 GN 11 vein

$13 \mathrm{R}-1,109 \mathrm{~cm}$ (Piece 12) 104.49 GN 11 vein

$13 \mathrm{R}-1,127 \mathrm{~cm}$ (Piece 14) 104.67 GN 11 vein

$13 \mathrm{R}-1,124 \mathrm{~cm}$ (Piece 14) 104.64 GN 11 vein

$13 \mathrm{R}-2,6 \mathrm{~cm}$ (Piece 1) $\quad 104.84$ GN 11 vein

13R-2, $1 \mathrm{~cm}$ (Piece 1) $\quad 104.79$ GN 11 vein

13R-2,31 cm (Piece 5) 105.09 GN II vein

$13 \mathrm{R}-2,35 \mathrm{~cm}$ (Piece 5) 105.13 GN 11 vein

$13 R-2,43 \mathrm{~cm}$ (Piece 5) 105.21 GN 11 vein

$13 R-2,60 \mathrm{~cm}$ (Piece 7) 105.38 GN 11 vein

$13 \mathrm{R}-2,62 \mathrm{~cm}$ (Piece 7) 105.40 GN 11 vein

$13 \mathrm{R}-2,68 \mathrm{~cm}$ (Piece 7) $105.46 \quad \mathrm{GN} \quad 11$ vein

13R-2, 74 cm (Piece 7) 105.52 GN 11 vein

$13 \mathrm{R}-2,57 \mathrm{~cm}$ (Piece 7) 105.35 GN 11 foliation

$13 \mathrm{R}-2.77 \mathrm{~cm}$ (Piece 7) 105.55 GN 11 vein

13R-2, $80 \mathrm{~cm}$ (Piece 7) $105.58 \mathrm{GN} \quad 11$ vein

$\begin{array}{lllll}13 \mathrm{R}-2,87 \mathrm{~cm} \text { (Piece 8) } & 105.65 & \mathrm{GN} & 11 & \text { vein } \\ 13 \mathrm{R}-2,97 \mathrm{~cm} \text { (Piece 8) } & 105.75 & \mathrm{GN} & 11 & \text { vein }\end{array}$

$\begin{array}{lllll}13 \mathrm{R}-2,97 \mathrm{~cm} \text { (Piece 8) } & 105.75 & \mathrm{GN} & 11 & \text { vein } \\ 13 \mathrm{R}-2,92 \mathrm{~cm} \text { (Piece 8) } & 105.70 & \mathrm{GN} & 11 & \text { foliation }\end{array}$

13R-2, $109 \mathrm{~cm}$ (Piece 9) 105.87 GN 11 vein

$13 \mathrm{R}-2,109 \mathrm{~cm}$ (Piece 9) $105.87 \mathrm{GN} \quad 11$ vein

$13 \mathrm{R}-2,115 \mathrm{~cm}$ (Piece 10) 105.93 GN 11 vein

$13 \mathrm{R}-2,123 \mathrm{~cm}$ (Piece 10) $106.01 \mathrm{GN}$ il vein

$13 R-2,131 \mathrm{~cm}$ (Piece 10) 106.09 GN 11 vein

$13 \mathrm{R}-2,140 \mathrm{~cm}$ (Piece 10) $106.18 \mathrm{GN} \quad 11$ vein

$13 R-2,130 \mathrm{~cm}$ (Piece 10) 106.08 GN 11 vein

13R-2, $138 \mathrm{~cm}$ (Piece 10) 106.16 GN 11 vein

$13 R-2,128 \mathrm{~cm}$ (Piece 10) 106.06 GN 11 vein

$13 \mathrm{R}-2,147 \mathrm{~cm}$ (Piece 10) 106.25 GN 11 vein

$13 \mathrm{R}-3,3 \mathrm{~cm}$ (Piece 1) 106.28 GN 11 vein

$13 \mathrm{R}-3,9 \mathrm{~cm}$ (Piece 1) 106.34 GN 11 vein

$13 \mathrm{R}-3,13 \mathrm{~cm}$ (Piece 1) 106.38 GN 11 vein

$13 \mathrm{R}-3,23 \mathrm{~cm}$ (Piece 1) 106.48 GN 11 vein

$13 \mathrm{R}-3,12 \mathrm{~cm}$ (Piece 1) $106.37 \mathrm{GN}$ il foliation

13R-3, $30 \mathrm{~cm}$ (Piece 2) $\quad 106.55$ GN 11 vein

$13 \mathrm{R}-3,32 \mathrm{~cm}$ (Piece 2) $106.57 \quad$ GN 11 vein

$13 \mathrm{R}-3,28 \mathrm{~cm}$ (Piece 2) $106.53 \mathrm{GN} 11$ vein

$13 R-3,27 \mathrm{~cm}$ (Piece 2) 106.52 GN 11 vein

$13 \mathrm{R}-3,42 \mathrm{~cm}$ (Piece 3 ) 106.67 GN 11 vein

13R-3, $48 \mathrm{~cm}$ (Piece 3) 106.73 GN 11 vein

$13 \mathrm{R}-3,47 \mathrm{~cm}$ (Piece 3) 106.72 GN 11 vein

13R-3, $45 \mathrm{~cm}$ (Piece 3) $106.70 \quad \mathrm{GN} 11$ vein

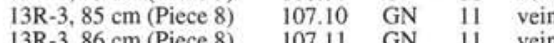

$13 \mathrm{R}-3,105 \mathrm{~cm}$ (Piece 11) $107.30 \mathrm{GN}$ it vein

13R-3, $109 \mathrm{~cm}$ (Piece 11) $107.34 \mathrm{GN}$.

$13 \mathrm{R}-3,105 \mathrm{~cm}$ (Piece 11$)$ 107.34 GN 11 vein

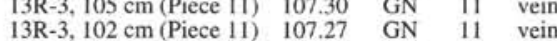

$\begin{array}{lllll}13 \mathrm{R}-3,102 \mathrm{~cm}(\text { Piece 11) } & 107.27 & \mathrm{GN} & 11 & \text { vein } \\ 13 \mathrm{R}-3,111 \mathrm{~cm} \text { (Piece 12) } & 107.36 & \mathrm{GN} & 11 & \text { vein }\end{array}$

$\begin{array}{lllll}13 \mathrm{R}-3,111 \mathrm{~cm}(\text { Piece 12) } & 107.36 & \mathrm{GN} & 11 & \text { vein } \\ 13 \mathrm{R}-3,110 \mathrm{~cm} \text { (Piece 12) } & 107.35 & \text { GN } & 11 & \text { vein }\end{array}$

13R-3, $118 \mathrm{~cm}$ (Piece 12) 107.43 GN 11 vein

13R-3, $120 \mathrm{~cm}$ (Piece 13) 107.45 GN 11 vein

$13 \mathrm{R}-3,131 \mathrm{~cm}$ (Piece 13) $107.56 \mathrm{GN}$ - 11 - foliation

13R-3, $3120 \mathrm{~cm}$ (Piece 13) $107.45 \mathrm{GN}$ il vein

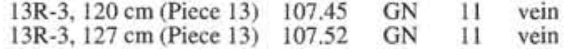

$\begin{array}{ll}-068.0 & 5 \\ -068.0 & 5 \\ -068.0 & 5 \\ -098.0 & 4 \\ -068.0 & 5 \\ -098.0 & 4 \\ -098.0 & 4\end{array}$

$\begin{array}{ll}52 & \text { J } \\ 52 & \mathrm{~J} \\ 52 & \mathrm{~J} \\ 45 & \mathrm{~J} \\ 52 & \mathrm{~J} \\ 45 & \mathrm{~J} \\ 45 & \mathrm{~J}\end{array}$

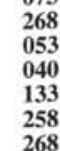

$\mathbf{5 0}$
$\mathbf{1 0}$
$\mathbf{7 0}$
$\mathbf{4 3}$
$\mathbf{9 0}$
$\mathbf{5 0}$
$\mathbf{5}$

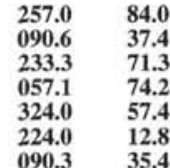

84.0
37.4
71.3
74.2
57.4
12.8
35.4 
Table 1 (continued).

\begin{tabular}{|c|c|c|c|c|c|c|c|c|}
\hline & Depth & & & & Vein & & Con & pined \\
\hline Sample number & (mbsf) & Lith. & Unit & Feature type & fill & Feat. \# & $\operatorname{str}\left({ }^{\circ}\right)$ & $\operatorname{dip}\left({ }^{\circ}\right)$ \\
\hline 14R-1, $67 \mathrm{~cm}$ (Piece 11) & 113.77 & GN & 11 & vein & S & 1 & 049 & 72 \\
\hline $14 \mathrm{R}-1,72 \mathrm{~cm}$ (Piece 11) & 113.82 & $\mathrm{GN}$ & 11 & vein & S & 2 & 010 & 71 \\
\hline $14 \mathrm{R}-1,66 \mathrm{~cm}$ (Piece 11$)$ & 113.76 & GN & 11 & vein & $\mathrm{s}$ & 3 & 350 & 47 \\
\hline $15 \mathrm{R}-1,46 \mathrm{~cm}$ (Piece 7) & 119.26 & $\mathrm{GN}$ & 11 & vein & S & 1 & 136 & 69 \\
\hline $15 \mathrm{R}-1,71 \mathrm{~cm}$ (Piece 12) & 119.51 & GN & 11 & ign contact & & 6 & 195 & 47 \\
\hline $15 \mathrm{R}-1,69 \mathrm{~cm}$ (Piece 12) & 119.49 & $\mathrm{GN}$ & 11 & vein & S & 3 & 037 & 86 \\
\hline 15R-1, $71 \mathrm{~cm}$ (Piece 12) & 119.51 & $\mathrm{GN}$ & 11 & vein & S & 4 & 263 & 34 \\
\hline $15 \mathrm{R}-1,76 \mathrm{~cm}$ (Piece 12) & 119.56 & $\mathrm{GN}$ & 11 & vein & A & 5 & 252 & 66 \\
\hline 17R-1, $24 \mathrm{~cm}$ (Piece 6) & 126.04 & GN & 11 & vein & $S$ & 1 & 114 & 14 \\
\hline 17R-1, $25 \mathrm{~cm}$ (Piece 6) & 126.05 & $\mathrm{GN}$ & 11 & vein & S & 2 & 121 & 90 \\
\hline 17R-1. $48 \mathrm{~cm}$ (Piece 10) & 126.28 & GN & 11 & vein & $\mathrm{S}$ & 1 & 224 & 34 \\
\hline $17 \mathrm{R}-1.50 \mathrm{~cm}$ (Piece 10) & 126.30 & GN & 11 & vein & S & 2 & 220 & 36 \\
\hline $17 \mathrm{R}-1,57 \mathrm{~cm}$ (Piece 10) & 126.37 & GN & 11 & vein & $\mathrm{s}$ & 5 & 188 & 83 \\
\hline $17 \mathrm{R}-1,61 \mathrm{~cm}$ (Piece 10) & 126.41 & $\mathrm{GN}$ & iI & vein & $\mathrm{s}$ & 7 & 187 & 86 \\
\hline $17 \mathrm{R}-1,60 \mathrm{~cm}$ (Piece 10) & 126.40 & $\mathrm{GN}$ & ii & vein & S & 6 & 022 & 88 \\
\hline 17R-1, $61 \mathrm{~cm}$ (Piece 10) & 126.41 & $\mathrm{GN}$ & 11 & vein & S & 8 & 005 & 90 \\
\hline $17 \mathrm{R}-1,52 \mathrm{~cm}$ (Piece 10) & 126.32 & GN & 11 & vein & $\mathrm{s}$ & 4 & 040 & 76 \\
\hline $17 \mathrm{R}-1,65 \mathrm{~cm}$ (Piece 10) & 126.45 & $\mathrm{GN}$ & 11 & foliation & & 10 & 260 & 84 \\
\hline $17 \mathrm{R}-1,53 \mathrm{~cm}$ (Piece 10) & 126.33 & GN & 11 & vein & $\mathrm{s}$ & 3 & 198 & 84 \\
\hline $17 \mathrm{R}-1,65 \mathrm{~cm}$ (Piece 10) & 126.45 & GN & 11 & vein & $\mathrm{s}$ & 9 & 173 & 82 \\
\hline $17 \mathrm{R}-1,90 \mathrm{~cm}$ (Piece 12) & 126.70 & $\mathrm{GN}$ & 11 & vein & S & 1 & 173 & 90 \\
\hline $17 \mathrm{R}-1,88 \mathrm{~cm}$ (Piece 12) & 126.68 & $\mathrm{GN}$ & i1 & vein & s & 2 & 059 & 80 \\
\hline 17R-1, $88 \mathrm{~cm}$ (Piece 12) & 126.68 & $\mathrm{GN}$ & 11 & vein & S & 3 & 338 & 31 \\
\hline 17R-1, $87 \mathrm{~cm}$ (Piece 12) & 126.67 & $\mathrm{GN}$ & 11 & vein & S & 4 & 150 & 78 \\
\hline $17 \mathrm{R}-1,95 \mathrm{~cm}$ (Piece 12) & 126.75 & GN & 11 & vein & S & 5 & 195 & 48 \\
\hline $17 \mathrm{R}-1,121 \mathrm{~cm}$ (Piece 14) & 127.01 & $\mathrm{GN}$ & 11 & vein & S & 1 & 192 & 31 \\
\hline $17 \mathrm{R}-1,125 \mathrm{~cm}$ (Piece 14) & 127.05 & GN & 11 & vein & S & 2 & 021 & 57 \\
\hline 17R-1, $114 \mathrm{~cm}$ (Piece 14) & 126.94 & GN & 11 & vein & $S$ & 3 & 080 & 59 \\
\hline $17 \mathrm{R}-1,125 \mathrm{~cm}$ (Piece 14) & 127.05 & $\mathrm{GN}$ & 11 & foliation & & 4 & 345 & 85 \\
\hline $17 \mathrm{R}-1,139 \mathrm{~cm}$ (Piece 16) & 127.19 & GN & 11 & vein & $\mathrm{s}$ & 1 & 350 & 60 \\
\hline $17 \mathrm{R}-1,142 \mathrm{~cm}$ (Piece 16) & 127.22 & $\mathrm{GN}$ & 11 & vein & S & 2 & 002 & 80 \\
\hline $17 \mathrm{R}-1,137 \mathrm{~cm}$ (Piece 16) & 127.17 & GN & 11 & vein & S & 3 & 338 & 76 \\
\hline 17R-2, $2 \mathrm{~cm}$ (Piece 1) & 127.26 & GN & 11 & vein & S & 1 & 160 & 66 \\
\hline $17 \mathrm{R}-2,22 \mathrm{~cm}$ (Piece 1) & 127.46 & GN & 11 & vein & S & 2 & 308 & 90 \\
\hline $17 \mathrm{R}-2,3 \mathrm{~cm}$ (Piece 1$)$ & 127.27 & GN & 11 & vein & $\mathrm{s}$ & 3 & 010 & 63 \\
\hline $17 \mathrm{R}-2,10 \mathrm{~cm}$ (Piece 1) & 127.34 & GN & 11 & vein & A & 4 & 293 & 88 \\
\hline $17 \mathrm{R}-2,10 \mathrm{~cm}$ (Piece 1) & 127.34 & GN & 11 & vein & S & 5 & 305 & 89 \\
\hline $17 \mathrm{R}-2,11 \mathrm{~cm}$ (Piece 1$)$ & 127.35 & $\mathrm{GN}$ & i1 & vein & $\mathrm{s}$ & 6 & 010 & 74 \\
\hline 17R-2, $12 \mathrm{~cm}$ (Piece 1) & 127.36 & $\mathrm{GN}$ & 11 & foliation & & 7 & 325 & 72 \\
\hline $17 \mathrm{R}-2,44 \mathrm{~cm}$ (Piece 5) & 127.68 & $\mathrm{GN}$ & 11 & vein & S & 1 & 196 & 61 \\
\hline 17R-2, $49 \mathrm{~cm}$ (Piece 6) & 127.73 & GN & 11 & vein & s & 1 & 178 & 86 \\
\hline I7R-2, $49 \mathrm{~cm}$ (Piece 6) & 127.73 & GN & 11 & vein & S & 2 & 172 & 88 \\
\hline 17R-2, $49 \mathrm{~cm}$ (Piece 6) & 127.73 & GN & 11 & vein & $\mathrm{s}$ & 3 & 186 & 59 \\
\hline $17 \mathrm{R}-2,64 \mathrm{~cm}$ (Piece 7) & 127.88 & GN & 11 & vein & A & i & 010 & 75 \\
\hline $17 \mathrm{R}-2,62 \mathrm{~cm}$ (Piece 7 ) & 127.86 & GN & 11 & vein & S & 2 & 296 & 22 \\
\hline $17 \mathrm{R}-2,66 \mathrm{~cm}$ (Piece 7) & 127.90 & GN & 11 & vein & S & 3 & 208 & 90 \\
\hline $17 \mathrm{R}-2,60 \mathrm{~cm}$ (Piece 7) & 127.84 & GN & 11 & vein & $\mathrm{S}$ & 6 & 308 & 32 \\
\hline $17 \mathrm{R}-2,88 \mathrm{~cm}$ (Piece 8 ) & 128.12 & GN & 11 & vein & S & 1 & 270 & 42 \\
\hline $17 \mathrm{R}-2,87 \mathrm{~cm}$ (Piece 8) & 128.11 & $\mathrm{GN}$ & 11 & vein & S & 2 & 159 & 90 \\
\hline $17 \mathrm{R}-2,72 \mathrm{~cm}$ (Piece 8) & 127.96 & $\mathrm{GN}$ & 11 & vein & S & 3 & 029 & 89 \\
\hline $17 \mathrm{R}-2.77 \mathrm{~cm}$ (Piece 8) & 128.01 & GN & 11 & vein & S & 4 & 011 & 61 \\
\hline $17 \mathrm{R}-2,80 \mathrm{~cm}$ (Piece 8) & 128.04 & GN & 11 & vein & S & 5 & 355 & 60 \\
\hline $17 \mathrm{R}-2,109 \mathrm{~cm}$ (Piece 12) & 128.33 & GN & 11 & vein & s & 1 & 175 & 85 \\
\hline $17 \mathrm{R}-2,112 \mathrm{~cm}$ (Piece 12) & 128.36 & GN & 11 & vein & $\mathrm{S}$ & 2 & 039 & 70 \\
\hline 17R-2, $114 \mathrm{~cm}$ (Piece 12) & 128.38 & GN & 11 & vein & $\mathrm{S}$ & 3 & 127 & 82 \\
\hline $17 \mathrm{R}-2,106 \mathrm{~cm}$ (Piece 12) & 128.30 & $\mathrm{GN}$ & 11 & vein & S & 4 & 185 & 77 \\
\hline 18R-1, $65 \mathrm{~cm}$ (Piece 10) & 131.55 & GN & 11 & vein & $\mathrm{s}$ & 1 & 189 & 84 \\
\hline $18 \mathrm{R}-1,68 \mathrm{~cm}$ (Piece 10) & 131.58 & GN & 11 & vein & S & 2 & 221 & 84 \\
\hline 18R-1, $65 \mathrm{~cm}$ (Piece 10) & 131.55 & GN & 11 & vein & S & 3 & 207 & 73 \\
\hline 18R-1, $79 \mathrm{~cm}$ (Piece 10) & 131.69 & GN & 11 & vein & $\mathrm{S}$ & 4 & 031 & 80 \\
\hline $18 \mathrm{R}-1,72 \mathrm{~cm}$ (Piece 10) & 131.62 & GN & 11 & vein & S & 6 & 009 & 78 \\
\hline $18 \mathrm{R}-1,80 \mathrm{~cm}$ (Piece 10$)$ & 131.70 & $\mathrm{GN}$ & 11 & foliation & & 7 & 075 & 89 \\
\hline $18 \mathrm{R}-1,125 \mathrm{~cm}$ (Piece 14) & 132.15 & $\mathrm{GN}$ & 11 & vein & $\mathrm{S}$ & $i$ & 047 & 72 \\
\hline $18 \mathrm{R}-1,127 \mathrm{~cm}$ (Piece 14) & 132.17 & GN & 11 & vein & $\mathrm{S}$ & 2 & 198 & 76 \\
\hline $18 \mathrm{R}-1,132 \mathrm{~cm}$ (Piece 14) & 132.22 & GN & 11 & vein & S & 3 & 024 & 24 \\
\hline $18 \mathrm{R}-1,134 \mathrm{~cm}$ (Piece 14) & 132.24 & GN & 11 & vein & S & 4 & 175 & 68 \\
\hline $18 \mathrm{R}-1,132 \mathrm{~cm}$ (Piece 14) & 132.22 & GN & 11 & foliation & & 5 & 150 & 86 \\
\hline $18 \mathrm{R}-2,0 \mathrm{~cm}$ (Piece 1) & 132.33 & GN & 11 & vein & $\mathrm{S}$ & 1 & 018 & 56 \\
\hline $18 \mathrm{R}-2,5 \mathrm{~cm}$ (Piece 1) & 132.38 & GN & 11 & vein & S & 2 & 107 & 80 \\
\hline $18 \mathrm{R}-2,9 \mathrm{~cm}$ (Piece 1) & 132.42 & GN & 11 & vein & $\mathrm{s}$ & 3 & 200 & 66 \\
\hline $18 \mathrm{R}-2,25 \mathrm{~cm}$ (Piece 4) & 132.58 & GN & 11 & ign contact & & 1 & 058 & 34 \\
\hline $18 \mathrm{R}-2,29 \mathrm{~cm}$ (Piece 4$)$ & 132.62 & GN & 11 & ign contact & & 2 & 058 & 34 \\
\hline $18 \mathrm{R}-2,30 \mathrm{~cm}$ (Piece 4) & 132.63 & GN & 11 & vein & S & 3 & 175 & 80 \\
\hline 18R-2, $23 \mathrm{~cm}$ (Piece 4) & 132.56 & GN & 11 & foliation & & 4 & 045 & 72 \\
\hline $18 \mathrm{R}-2,76 \mathrm{~cm}$ (Piece 11) & 133.09 & GN & 11 & vein & C & i & 169 & 27 \\
\hline $18 \mathrm{R}-2,106 \mathrm{~cm}$ (Piece 16) & 133.39 & GN & 11 & vein & $\mathrm{C}$ & 1 & 146 & 40 \\
\hline $18 \mathrm{R}-2,107 \mathrm{~cm}$ (Piece 16) & 133.40 & GN & 11 & vein & $\mathrm{C}$ & 2 & 221 & 19 \\
\hline 18R-2, $104 \mathrm{~cm}$ (Piece 16) & 133.37 & GN & 11 & vein & $\mathrm{C}$ & 3 & 170 & 33 \\
\hline $18 \mathrm{R}-2,110 \mathrm{~cm}$ (Piece 16) & 133.43 & GN & 11 & vein & $\mathrm{S}$ & 4 & 054 & 82 \\
\hline $19 \mathrm{R}-1,30 \mathrm{~cm}$ (Piece 7) & 140.80 & $\mathrm{GN}$ & 11 & vein & $\mathrm{C}$ & 1 & 189 & 86 \\
\hline 19R-1, $32 \mathrm{~cm}$ (Piece 7) & 140.82 & GN & 11 & vein & $\mathrm{C}$ & 2 & 188 & 73 \\
\hline 19R-1, $37 \mathrm{~cm}$ (Piece 7) & 140.87 & GN & 11 & vein & S & 4 & 215 & 41 \\
\hline 19R-1, $34 \mathrm{~cm}$ (Piece 7) & 140.84 & GN & 11 & foliation & & 5 & 090 & 90 \\
\hline $19 \mathrm{R}-1,77 \mathrm{~cm}$ (Piece 12) & 141.27 & B & 12 & dike marg & & 3 & 336 & 87 \\
\hline $19 \mathrm{R}-1,70 \mathrm{~cm}$ (Piece 12) & 141.20 & B & 12 & vein & $\mathrm{C}$ & 1 & 001 & 82 \\
\hline 19R-1, $74 \mathrm{~cm}$ (Piece 12) & 141.24 & B & 12 & vein & $\mathrm{C}$ & 2 & 240 & 49 \\
\hline 19R-1, $84 \mathrm{~cm}$ (Piece 13) & 141.34 & B & 12 & vein & $\mathrm{s}$ & 3 & 007 & 61 \\
\hline 19R-1, $87 \mathrm{~cm}$ (Piece 13) & 141.37 & B & 12 & vein & $\mathrm{s}$ & 1 & 134 & 32 \\
\hline
\end{tabular}


Table 1 (continued).

\begin{tabular}{|c|c|c|c|c|c|c|c|c|c|c|c|c|c|c|c|}
\hline \multirow[b]{2}{*}{ Sample number } & \multirow{2}{*}{$\begin{array}{l}\text { Depth } \\
\text { (mbsf) }\end{array}$} & \multirow[b]{2}{*}{ Lith. } & \multirow[b]{2}{*}{ Unit } & \multirow[b]{2}{*}{ Feature type } & \multirow{2}{*}{$\begin{array}{l}\text { Vein } \\
\text { fill }\end{array}$} & \multirow[b]{2}{*}{ Feat. \# } & \multicolumn{2}{|c|}{ Combined } & \multicolumn{2}{|c|}{ Stable mag. rem. } & \multirow[b]{2}{*}{$\begin{array}{c}\text { Meas. } \\
\text { by }\end{array}$} & \multicolumn{2}{|c|}{ Rest (D only) } & \multicolumn{2}{|c|}{ Rest $(\mathrm{D}+\mathrm{I})$} \\
\hline & & & & & & & $\operatorname{str}\left({ }^{\circ}\right)$ & $\operatorname{dip}\left({ }^{\circ}\right)$ & $\operatorname{dec}\left({ }^{\circ}\right)$ & $\operatorname{inc}\left({ }^{\circ}\right)$ & & $\operatorname{str}\left({ }^{\circ}\right)$ & $\operatorname{dip}\left({ }^{\circ}\right)$ & $\operatorname{str}\left({ }^{\circ}\right)$ & $\operatorname{dip}\left({ }^{\circ}\right)$ \\
\hline 19R-1,91 cm (Piece 13) & 141.41 & B & 12 & vein & $\mathrm{S}$ & 2 & 221 & 32 & & & & & & & \\
\hline $20 \mathrm{R}-1,20 \mathrm{~cm}$ (Piece 4) & 145.80 & GN & 13 & vein & $\mathrm{s}$ & 2 & 180 & 75 & & & & & & & \\
\hline $20 \mathrm{R}-1,53 \mathrm{~cm}$ (Piece I0) & 146.13 & GN & 13 & vein & $S$ & 1 & 027 & 74 & -0.49 .4 & 44.1 & $\mathrm{~S}$ & 076 & 74 & 255.4 & 67.6 \\
\hline $20 \mathrm{R}-1,57 \mathrm{~cm}$ (Piece 10) & 146.17 & GN & 13 & vein & $\mathrm{S}$ & 2 & 100 & 55 & -049.4 & 44.1 & S & 149 & 55 & 135.5 & 80.0 \\
\hline $20 \mathrm{R}-1,53 \mathrm{~cm}$ (Piece 10) & 146.13 & $\mathrm{GN}$ & 13 & vein & $\mathrm{S}$ & 3 & 306 & 64 & -049.4 & 44.1 & $\mathrm{~s}$ & 355 & 64 & 013.7 & 67.2 \\
\hline $20 \mathrm{R}-1,55 \mathrm{~cm}$ (Piece 10) & 146.15 & $\mathrm{GN}$ & 13 & vein & $\mathrm{S}$ & 4 & 008 & 65 & -049.4 & 44.1 & S & 057 & 65 & 240.0 & 80.9 \\
\hline $20 \mathrm{R}-1,88 \mathrm{~cm}$ (Piece 15) & 146.48 & GN & 13 & vein & S & i & 005 & 63 & 056.0 & 32 & $\mathrm{~J}$ & 309 & 63 & 324.1 & 43.8 \\
\hline 20R-1, $92 \mathrm{~cm}$ (Piece 15) & 146.52 & $\mathrm{GN}$ & 13 & vein & $S$ & 2 & 013 & 63 & 056.0 & 32 & $\mathrm{j}$ & 317 & 63 & 333.1 & 46.9 \\
\hline $20 \mathrm{R}-1,121 \mathrm{~cm}$ (Piece 19) & 146.81 & GN & 13 & vein & $\mathrm{s}$ & 1 & 341 & 80 & & & & & & & \\
\hline $20 \mathrm{R}-1,115 \mathrm{~cm}$ (Piece 19) & 146.75 & $\mathrm{GN}$ & 13 & vein & $\mathrm{s}$ & 2 & 188 & 87 & & & & & & & \\
\hline $20 \mathrm{R}-1,133 \mathrm{~cm}$ (Piece 21) & 146.93 & GN & 13 & vein & $\mathrm{S}$ & 1 & 026 & 76 & & & & & & & \\
\hline $20 \mathrm{R}-1,129 \mathrm{~cm}$ (Piece 21) & 146.89 & GN & 13 & vein & $\mathrm{S}$ & 2 & 034 & 73 & & & & & & & \\
\hline $20 \mathrm{R}-2,0 \mathrm{~cm}$ (Piece $\mathrm{I})$ & 147.03 & GN & 13 & vein & $\mathrm{S}$ & 1 & 167 & 75 & & & & & & & \\
\hline 20R-2, $16 \mathrm{~cm}$ (Piece 2) & 147.19 & GN & 13 & vein & $\mathrm{S}$ & 1 & 149 & 79 & & & & & & & \\
\hline 20R-2, $33 \mathrm{~cm}$ (Piece 4) & 147.36 & GN & 13 & vein & $\mathrm{S}$ & $\mathrm{i}$ & 185 & 32 & & & & & & & \\
\hline $20 \mathrm{R}-2,35 \mathrm{~cm}$ (Piece 4$)$ & 147.38 & GN & 13 & vein & $\mathrm{s}$ & 2 & 205 & 36 & & & & & & & \\
\hline 20R-2, $38 \mathrm{~cm}$ (Piece 4) & 147.41 & GN & 13 & vein & $\mathrm{S}$ & 3 & 347 & 85 & & & & & & & \\
\hline 20R-2, $38 \mathrm{~cm}$ (Piece 4) & 147.41 & GN & 13 & vein & $\mathrm{S}$ & 4 & 170 & 90 & & & & & & & \\
\hline 20R-3, $50 \mathrm{~cm}$ (Piece 9) & 147.94 & $\mathrm{GN}$ & 13 & vein & $\mathrm{S}$ & 1 & 190 & 74 & Unstable & & $\mathrm{S}$ & & & & \\
\hline $20 \mathrm{R}-3,55 \mathrm{~cm}$ (Piece 9) & 147.99 & GN & 13 & vein & $\mathrm{s}$ & 2 & 185 & 52 & Unstable & & $\mathrm{s}$ & & & & \\
\hline 20R-3.63 cm (Piece 10) & 148.07 & GN & 13 & vein & $\mathrm{S}$ & $i$ & 179 & 86 & & & & & & & \\
\hline 20R-3, $64 \mathrm{~cm}$ (Piece 10) & 148.08 & GN & 13 & vein & A & 2 & 175 & 90 & & & & & & & \\
\hline $20 \mathrm{R}-3,79 \mathrm{~cm}$ (Piece 12) & 148.23 & GN & 13 & vein & $\mathrm{S}$ & 3 & 352 & 68 & & & & & & & \\
\hline $20 \mathrm{R}-3,80 \mathrm{~cm}$ (Piece 12) & 148.24 & GN & 13 & vein & $\mathrm{s}$ & i & 270 & 5 & & & & & & & \\
\hline 20R-3, $104 \mathrm{~cm}$ (Piece 15) & 148.48 & $\mathrm{GN}$ & 13 & vein & $\mathrm{s}$ & $\mathrm{i}$ & 177 & 65 & & & & & & & \\
\hline $20 \mathrm{R}-3,119 \mathrm{~cm}$ (Piece 17) & 148.63 & GN & 13 & vein & $\mathrm{S}$ & $i$ & 142 & 84 & 049.0 & -31 & $\mathrm{C}$ & 093 & 84 & 094.0 & 48.5 \\
\hline $20 \mathrm{R}-3,121 \mathrm{~cm}$ (Piece 17) & 148.65 & $\mathrm{GN}$ & 13 & vein & $\mathrm{S}$ & 2 & 015 & 82 & 049.0 & -31 & $\mathrm{C}$ & 326 & 82 & 147.1 & 77.9 \\
\hline $20 \mathrm{R}-3,125 \mathrm{~cm}$ (Piece 17) & 148.69 & GN & 13 & vein & $\mathrm{S}$ & 3 & 331 & 68 & 049.0 & -31 & C & 282 & 68 & 101.4 & 77.1 \\
\hline 20R-3, $124 \mathrm{~cm}$ (Piece 17) & 148.68 & $\mathrm{GN}$ & 13 & vein & $\mathrm{s}$ & 4 & 023 & 76 & 049.0 & $-3 I$ & C & 334 & 76 & 150.8 & 87.1 \\
\hline $20 \mathrm{R}-3,128 \mathrm{~cm}$ (Piece 17 ) & 148.72 & $\mathrm{GN}$ & 13 & vein & $\mathrm{s}$ & 5 & 187 & 70 & 049.0 & -31 & $\mathrm{C}$ & 138 & 70 & 155.9 & 49.9 \\
\hline
\end{tabular}

Table 2. Structural data from Site 895.

\begin{tabular}{|c|c|c|c|c|c|c|c|c|c|c|c|c|c|}
\hline \multirow[b]{2}{*}{ Sample number } & \multirow{2}{*}{$\begin{array}{l}\text { Depth } \\
\text { (mbsf) }\end{array}$} & \multirow[b]{2}{*}{ Lith. } & \multirow[b]{2}{*}{ Feature type } & \multirow[b]{2}{*}{ Feat. \# } & \multicolumn{2}{|c|}{ Combined } & \multicolumn{2}{|c|}{ Stable mag. rem. } & \multirow[b]{2}{*}{$\begin{array}{c}\text { Meas. } \\
\text { by }\end{array}$} & \multicolumn{2}{|c|}{ Rest (D only) } & \multicolumn{2}{|c|}{ Rest (D+I) } \\
\hline & & & & & $\operatorname{str}\left({ }^{\circ}\right)$ & $\operatorname{dip}\left({ }^{\circ}\right)$ & $\operatorname{dec}\left({ }^{\circ}\right)$ & $\operatorname{inc}\left({ }^{\circ}\right)$ & & $\operatorname{str}\left({ }^{\circ}\right)$ & $\operatorname{dip}\left({ }^{\circ}\right)$ & $\operatorname{str}\left({ }^{\circ}\right)$ & $\operatorname{dip}\left({ }^{\circ}\right)$ \\
\hline \multicolumn{14}{|l|}{ Hole 895B- } \\
\hline IR-1, $60 \mathrm{~cm}$ (Piece 9) & 0.60 & $\mathrm{HZ}$ & foliation & 3 & 300 & 81 & -063.4 & 39.3 & $\mathrm{~S}(+\mathrm{C})$ & 003.4 & 81 & 007.9 & 84.5 \\
\hline IR-1. $65 \mathrm{~cm}$ (Piece 9) & 0.65 & $\mathrm{HZ}$ & vein & 1 & 198 & 66 & -063.4 & 39.3 & $\mathrm{~s}$ & 261.4 & 66 & 255.0 & 31.9 \\
\hline $1 \mathrm{R}-1,79 \mathrm{~cm}$ (Piece 9) & 0.79 & $\mathrm{HZ}$ & vein & 2 & 173 & 65 & -063.4 & 39.3 & $\mathrm{~S}$ & 236.4 & 65 & 217.2 & 39.0 \\
\hline \multicolumn{14}{|l|}{ Hole $895 \mathrm{C}$ - } \\
\hline $1 \mathrm{R}-1,32 \mathrm{~cm}$ (Piece 5) & 0.32 & $\mathrm{HZ}$ & foliation & 1 & 005 & 60 & & & & & & & \\
\hline $1 \mathrm{R}-1,61 \mathrm{~cm}$ (Piece 9) & 0.61 & $\mathrm{HZ}$ & foliation & 1 & 305 & 25 & & & & & & & \\
\hline $1 \mathrm{R}-1,109 \mathrm{~cm}$ (Piece 17) & 1.09 & $\mathrm{HZ}$ & foliation & 1 & 180 & 65 & & & & & & & \\
\hline 1R-1, $119 \mathrm{~cm}$ (Piece 18) & 1.19 & $\mathrm{HZ}$ & foliation & 1 & 140 & 62 & & & & & & & \\
\hline $3 \mathrm{R}-1,138 \mathrm{~cm}$ (Piece 22) & 19.68 & $\mathrm{HZ}$ & foliation & i & 000 & 90 & -012.8 & 45.7 & $\mathrm{~S}$ & 012.8 & 90 & 189.7 & 81.6 \\
\hline $3 \mathrm{R}-2,14 \mathrm{~cm}$ (Piece 2) & 19.89 & $\mathrm{HZ}$ & vein & 1 & 192 & 41 & & & & & & & \\
\hline $3 \mathrm{R}-2,16 \mathrm{~cm}$ (Piece 2) & 19.91 & $\mathrm{HZ}$ & foliation & 2 & 345 & 56 & & & & & & & \\
\hline $4 \mathrm{R}-1,20 \mathrm{~cm}$ (Piece 4) & 28.10 & DN & vein & 1 & 177 & 85 & & & & & & & \\
\hline $4 \mathrm{R}-2,54 \mathrm{~cm}$ (Piece 1) & 29.52 & IMP DN & vein & 1 & 012 & 22 & & & & & & & \\
\hline $4 \mathrm{R}-2,49 \mathrm{~cm}$ (Piece I) & 29.47 & IMP DN & vein & 2 & 184 & 40 & & & & & & & \\
\hline $4 \mathrm{R}-2,28 \mathrm{~cm}$ (Piece I) & 29.26 & IMP DN & vein & 3 & 168 & 41 & & & & & & & \\
\hline $4 \mathrm{R}-2,34 \mathrm{~cm}$ (Piece 1) & 29.32 & IMP DN & vein & 4 & 161 & 67 & & & & & & & \\
\hline $4 \mathrm{R}-2,16 \mathrm{~cm}$ (Piece 1$)$ & 29.14 & IMP DN & vein & 5 & 290 & 77 & & & & & & & \\
\hline $4 \mathrm{R}-2,94 \mathrm{~cm}$ (Piece 6) & 29.92 & IMP DN & vein & 1 & 044 & 39 & 056.0 & 44.0 & C & 348.0 & 39 & 032.5 & 46.9 \\
\hline 4R-2, $98 \mathrm{~cm}$ (Piece 6) & 29.96 & IMP DN & vein & 2 & 020 & 33 & 056.0 & 44.0 & $\mathrm{C}$ & 324.0 & 33 & 032.9 & 31.7 \\
\hline $4 \mathrm{R}-2,101 \mathrm{~cm}$ (Piece 6) & 29.99 & IMP DN & vein & 3 & 024 & 31 & 056.0 & 44.0 & $\mathrm{C}$ & 328.0 & 31 & 037.3 & 33.3 \\
\hline 4R-3, $41 \mathrm{~cm}$ (Piece 4) & 30.89 & $\mathrm{HZ}$ & vein & 1 & 179 & 85 & & & & & & & \\
\hline $4 \mathrm{R}-3,41 \mathrm{~cm}$ (Piece 4) & 30.89 & $\mathrm{HZ}$ & foliation & 2 & 020 & 90 & & & & & & & \\
\hline \multicolumn{14}{|l|}{ Hole 895D- } \\
\hline $2 \mathrm{R}-1.47 \mathrm{~cm}$ (Piece 6) & 16.47 & $\mathrm{HZ}$ & vein & 1 & 188 & 12 & -138.7 & 37.8 & $\mathrm{~S}$ & 326.7 & 12 & 068.5 & 28.2 \\
\hline $2 \mathrm{R}-1,55 \mathrm{~cm}$ (Piece 6) & 16.55 & $\mathrm{HZ}$ & foliation & 2 & 080 & 87 & -138.7 & 37.8 & $\mathrm{~S}$ & 218.7 & 87 & 212.4 & 67.3 \\
\hline $2 \mathrm{R}-2,6 \mathrm{~cm}$ (Piece 1 ) & 17.48 & $\mathrm{HZ}$ & vein & 1 & 167 & 34 & & & & & & & \\
\hline $2 \mathrm{R}-2.7 \mathrm{~cm}$ (Piece 1) & 17.49 & $\mathrm{HZ}$ & vein & 2 & 342 & 76 & & & & & & & \\
\hline $2 \mathrm{R}-2,15 \mathrm{~cm}$ (Piece 1) & 17.57 & $\mathrm{HZ}$ & vein & 3 & 171 & 35 & & & & & & & \\
\hline $2 \mathrm{R}-2,11 \mathrm{~cm}$ (Piece 1) & 17.53 & $\mathrm{HZ}$ & foliation & 4 & 190 & 52 & & & & & & & \\
\hline 2R-2. $6 \mathrm{~cm}$ (Piece 1) & 17.48 & $\mathrm{HZ}$ & ign contact & 5 & 190 & 70 & & & & & & & \\
\hline $2 \mathrm{R}-2,42 \mathrm{~cm}$ (Piece 3 ) & 17.84 & $\mathrm{HZ}$ & $\begin{array}{l}\text { foliation } \\
\text { follat }\end{array}$ & 1 & 235 & 72 & 109.1 & -34.2 & $\mathrm{~S}(+\mathrm{C})$ & 125.9 & 72 & 143.9 & 43.7 \\
\hline $2 \mathrm{R}-2,66 \mathrm{~cm}$ (Piece 5 ) & 18.08 & $\mathrm{HZ}$ & foliation & 1 & 050 & 70 & & & & & & & \\
\hline $2 \mathrm{R}-2,80 \mathrm{~cm}$ (Piece 6) & 18.22 & $\mathrm{HZ}$ & foliation & 1 & 045 & 72 & 019.0 & -10.0 & $\mathrm{P}$ & 026.0 & 72 & 020.8 & 66.2 \\
\hline $3 \mathrm{R}-1,35 \mathrm{~cm}$ (Piece 6) & 26.35 & $\mathrm{HZ}$ & vein & 1 & 174 & 67 & 116.3 & -23.0 & $\mathrm{~S}$ & 057.7 & 67 & 046.0 & 45.0 \\
\hline $3 \mathrm{R}-1,75 \mathrm{~cm}$ (Piece 9) & 26.75 & $\mathrm{HZ}$ & vein & i & 000 & 90 & & & & & & & \\
\hline $3 \mathrm{R}-1,73 \mathrm{~cm}$ (Piece 9) & 26.73 & $\mathrm{HZ}$ & vein netwk & 2 & 010 & 90 & & & & & & & \\
\hline 3R-1, $75 \mathrm{~cm}$ (Piece 9) & 26.75 & $\mathrm{HZ}$ & foliation & 3 & 334 & 86 & & & & & & & \\
\hline $3 \mathrm{R}-1,92 \mathrm{~cm}$ (Piece 11) & 26.92 & $\mathrm{HZ}$ & vein & 1 & 080 & 72 & & & & & & & \\
\hline $3 \mathrm{R}-1,112 \mathrm{~cm}$ (Piece 13) & 27.12 & $\mathrm{HZ}$ & vein & i & 170 & 18 & & & & & & & \\
\hline $3 \mathrm{R}-1,113 \mathrm{~cm}$ (Piece 13) & 27.13 & $\mathrm{HZ}$ & vein & 3 & 309 & 43 & & & & & & & \\
\hline $3 \mathrm{R}-1,128 \mathrm{~cm}$ (Piece 15) & 27.28 & $\mathrm{HZ}$ & vein & 1 & 351 & 80 & & & & & & & \\
\hline $3 \mathrm{R}-1,134 \mathrm{~cm}$ (Piece 15) & 27.34 & $\mathrm{HZ}$ & vein & 2 & 023 & 19 & & & & & & & \\
\hline $3 \mathrm{R}-1,125 \mathrm{~cm}$ (Piece 15) & 27.25 & $\mathrm{HZ}$ & vein & 3 & 352 & 85 & & & & & & & \\
\hline $3 \mathrm{R}-1.137 \mathrm{~cm}$ (Piece 15) & 27.37 & $\mathrm{HZ}$ & vein & 5 & 356 & 32 & & & & & & & \\
\hline $4 \mathrm{R}-2,4 \mathrm{~cm}$ (Piece 1) & 35.63 & $\mathrm{HZ}$ & vein & 1 & 185 & 10 & & & & & & & \\
\hline $4 \mathrm{R}-2,7 \mathrm{~cm}$ (Piece 1) & 35.66 & $\mathrm{HZ}$ & foliation & 2 & 330 & 68 & & & & & & & \\
\hline
\end{tabular}


Table 2 (continued).

\begin{tabular}{|c|c|c|c|c|c|c|c|c|}
\hline & Depth & & & & Con & ined & Stable n & ig. rem. \\
\hline Sample number & (mbsf) & Lith. & Feature type & Feat. \# & $\operatorname{str}\left({ }^{\circ}\right)$ & $\operatorname{dip}\left({ }^{\circ}\right)$ & $\operatorname{dec}\left({ }^{\circ}\right)$ & $\operatorname{inc}\left({ }^{\circ}\right)$ \\
\hline 4R-2, $23 \mathrm{~cm}$ (Piece 4) & 35.82 & $\mathrm{HZ}$ & foliation & 1 & 265 & 88 & & \\
\hline 4R-2, $28 \mathrm{~cm}$ (Piece 4) & 35.87 & $\mathrm{HZ}$ & vein & 2 & 296 & 22 & & \\
\hline $4 \mathrm{R}-2,41 \mathrm{~cm}$ (Piece 6) & 36.00 & $\mathrm{HZ}$ & vein & 1 & 148 & 34 & & \\
\hline $4 \mathrm{R}-2,45 \mathrm{~cm}$ (Piece 6) & 36.04 & $\mathrm{HZ}$ & vein & 2 & 320 & 53 & & \\
\hline 4R-2, $38 \mathrm{~cm}$ (Piece 6) & 35.97 & $\mathrm{HZ}$ & vein & 3 & 000 & 0 & & \\
\hline $4 \mathrm{R}-2,42 \mathrm{~cm}$ (Piece 6) & 36.01 & $\mathrm{HZ}$ & foliation & 4 & 175 & 80 & & \\
\hline 4R-2, $103 \mathrm{~cm}$ (Piece 15) & 36.62 & $\mathrm{HZ}$ & vein & 2 & 180 & 5 & & \\
\hline $4 \mathrm{R}-2,123 \mathrm{~cm}$ (Piece 18) & 36.82 & $\mathrm{HZ}$ & vein netwk & 1 & 010 & 75 & & \\
\hline $4 \mathrm{R}-2,132 \mathrm{~cm}$ (Piece 18) & 36.91 & $\mathrm{HZ}$ & foliation & 2 & 090 & 90 & & \\
\hline $4 \mathrm{R}-3,5 \mathrm{~cm}$ (Piece 1) & 37.04 & $\mathrm{HZ}$ & vein & 1 & 350 & 70 & 021.0 & -9.0 \\
\hline $4 \mathrm{R}-3,18 \mathrm{~cm}$ (Piece 2$)$ & 37.17 & IMP DN & vein & i & 200 & 57 & 053.0 & -11.0 \\
\hline $4 \mathrm{R}-3,51 \mathrm{~cm}$ (Piece 6) & 37.50 & $\mathrm{HZ}$ & vein & 1 & 350 & 50 & & \\
\hline $4 \mathrm{R}-3,50 \mathrm{~cm}$ (Piece 6) & 37.49 & $\mathrm{HZ}$ & vein & 2 & 200 & 37 & & \\
\hline $4 \mathrm{R}-3,95 \mathrm{~cm}$ (Piece 11 ) & 37.94 & $\mathrm{HZ}$ & foliation & 1 & 180 & 70 & & \\
\hline $4 \mathrm{R}-3,109 \mathrm{~cm}$ (Piece 12) & 38.08 & $\mathrm{HZ}$ & foliation & 1 & 285 & 73 & 048.8 & 38.3 \\
\hline $4 \mathrm{R}-3,121 \mathrm{~cm}$ (Piece 13) & 38.20 & $\mathrm{HZ}$ & vein & i & 180 & 15 & & \\
\hline $4 \mathrm{R}-3,127 \mathrm{~cm}$ (Piece 14) & 38.26 & $\mathrm{HZ}$ & vein & 1 & 299 & 44 & & \\
\hline $4 \mathrm{R}-3,128 \mathrm{~cm}$ (Piece 14) & 38.27 & $\mathrm{HZ}$ & vein & 2 & 299 & 44 & & \\
\hline $4 \mathrm{R}-3,135 \mathrm{~cm}$ (Piece 15) & 38.34 & $\mathrm{HZ}$ & vein & i & 000 & 30 & & \\
\hline $4 \mathrm{R}-3,136 \mathrm{~cm}$ (Piece 15) & 38.35 & $\mathrm{HZ}$ & vein & 2 & 000 & 30 & & \\
\hline $4 \mathrm{R}-4,14 \mathrm{~cm}$ (Piece 2$)$ & 38.59 & $\mathrm{HZ}$ & vein & 1 & 000 & 10 & & \\
\hline $4 \mathrm{R}-4,18 \mathrm{~cm}$ (Piece 2) & 38.63 & $\mathrm{HZ}$ & foliation & 2 & 295 & 74 & & \\
\hline $4 \mathrm{R}-4,32 \mathrm{~cm}$ (Piece 4) & 38.77 & $\mathrm{HZ}$ & vein & 1 & 175 & 65 & & \\
\hline $4 \mathrm{R}-4,33 \mathrm{~cm}$ (Piece 4) & 38.78 & $\mathrm{HZ}$ & vein & 3 & 270 & 5 & & \\
\hline $4 \mathrm{R}-4,43 \mathrm{~cm}$ (Piece 5) & 38.88 & $\mathrm{HZ}$ & vein & 1 & 355 & 75 & & \\
\hline $4 \mathrm{R}-4,45 \mathrm{~cm}$ (Piece 5 ) & 38.90 & $\mathrm{HZ}$ & vein & 2 & 170 & 55 & & \\
\hline $4 \mathrm{R}-4,45 \mathrm{~cm}$ (Piece 5 ) & 38.90 & $\mathrm{HZ}$ & vein & 3 & 253 & 31 & & \\
\hline $4 \mathrm{R}-4,96 \mathrm{~cm}$ (Piece 14$)$ & 39.41 & $\mathrm{HZ}$ & vein & 1 & 000 & 65 & 265.0 & 41.0 \\
\hline $4 \mathrm{R}-4,99 \mathrm{~cm}$ (Piece 14) & 39.44 & $\mathrm{HZ}$ & vein & 2 & 026 & 22 & 265.0 & 41.0 \\
\hline $4 \mathrm{R}-5,3 \mathrm{~cm}$ (Piece 1) & 39.76 & $\mathrm{HZ}$ & vein netwk & 1 & 125 & 45 & 023.3 & 24.1 \\
\hline $4 \mathrm{R}-5,10 \mathrm{~cm}$ (Piece 1) & 39.83 & $\mathrm{HZ}$ & foliation & 2 & 190 & 40 & 023.3 & 24.1 \\
\hline $5 \mathrm{R}-1,45 \mathrm{~cm}$ (Piece 8$)$ & 43.75 & $\mathrm{HZ}$ & vein & 1 & 171 & 66 & -088.6 & -6.4 \\
\hline $5 \mathrm{R}-1,49 \mathrm{~cm}$ (Piece 8 ) & 43.79 & $\mathrm{HZ}$ & vein & 2 & 160 & 56 & -088.6 & -6.4 \\
\hline $5 \mathrm{R}-1,48 \mathrm{~cm}$ (Piece 8 ) & 43.78 & $\mathrm{HZ}$ & foliation & 3 & 310 & 42 & -088.6 & -6.4 \\
\hline $5 \mathrm{R}-1.123 \mathrm{~cm}$ (Piece 18) & 44.53 & $\mathrm{HZ}$ & vein & 1 & 005 & 41 & & \\
\hline $5 \mathrm{R}-1,115 \mathrm{~cm}$ (Piece 18) & 44.45 & $\mathrm{HZ}$ & vein & 2 & 084 & 51 & & \\
\hline $5 \mathrm{R}-1,120 \mathrm{~cm}$ (Piece 18) & 44.50 & $\mathrm{HZ}$ & vein & 3 & 249 & 44 & & \\
\hline $5 \mathrm{R}-1,122 \mathrm{~cm}$ (Piece 18) & 44.52 & $\mathrm{HZ}$ & foliation & 4 & 240 & 67 & & \\
\hline $5 \mathrm{R}-2,5 \mathrm{~cm}$ (Piece 2$)$ & 44.79 & $\mathrm{HZ}$ & vein & 1 & 349 & 30 & & \\
\hline $5 \mathrm{R}-2,6 \mathrm{~cm}$ (Piece 2 ) & 44.80 & $\mathrm{HZ}$ & vein & 2 & 341 & 33 & & \\
\hline $5 \mathrm{R}-2,10 \mathrm{~cm}$ (Piece 2) & 44.84 & $\mathrm{HZ}$ & vein & 3 & 043 & 27 & & \\
\hline $5 \mathrm{R}-2,23 \mathrm{~cm}$ (Piece 4) & 44.97 & $\mathrm{HZ}$ & vein & 1 & 189 & 85 & & \\
\hline $5 \mathrm{R}-2,22 \mathrm{~cm}$ (Piece 4) & 44.96 & $\mathrm{HZ}$ & vein & 2 & 358 & 86 & & \\
\hline $5 \mathrm{R}-2,23 \mathrm{~cm}$ (Piece 4) & 44.97 & $\mathrm{HZ}$ & vein & 3 & 001 & 82 & & \\
\hline $5 \mathrm{R}-2,50 \mathrm{~cm}$ (Piece 7) & 45.24 & $\mathrm{HZ}$ & vein & 1 & 199 & 81 & & \\
\hline $5 \mathrm{R}-2,51 \mathrm{~cm}$ (Piece 7) & 45.25 & $\mathrm{HZ}$ & vein & 2 & 197 & 56 & & \\
\hline $5 R-2,111 \mathrm{~cm}$ (Piece 14) & 45.85 & $\mathrm{HZ}$ & vein & 1 & 191 & 79 & 162.7 & -3.1 \\
\hline $5 \mathrm{R}-2,103 \mathrm{~cm}$ (Piece 14) & 45.77 & $\mathrm{HZ}$ & foliation & 2 & 000 & 58 & 162.7 & -3.1 \\
\hline $6 \mathrm{R}-1,27 \mathrm{~cm}$ (Piece 5) & 55.27 & $\mathrm{HZ}$ & vein & 1 & 340 & 62 & & \\
\hline $6 \mathrm{R}-1,65 \mathrm{~cm}$ (Piece 10) & 55.65 & $\mathrm{HZ}$ & vein & i & 042 & 13 & & \\
\hline $6 \mathrm{R}-1,74 \mathrm{~cm}$ (Piece 11$)$ & 55.74 & $\mathrm{HZ}$ & vein & i & 186 & 21 & 176.0 & 61.0 \\
\hline $7 \mathrm{R}-1,18 \mathrm{~cm}$ (Piece 4) & 64.78 & ROD G & foliation & 1 & 280 & 45 & & \\
\hline $7 \mathrm{R}-1,61 \mathrm{~cm}$ (Piece 10) & 65.21 & IMP DN & vein & 1 & 190 & 40 & 241.0 & -15.0 \\
\hline $7 \mathrm{R}-1.132 \mathrm{~cm}$ (Piece 20) & 65.92 & $\mathrm{HZ}$ & foliation & 1 & 280 & 78 & 036.7 & 59.4 \\
\hline $7 \mathrm{R}-2,13 \mathrm{~cm}$ (Piece 2) & 66.20 & $\mathrm{HZ}$ & foliation & 1 & 095 & 87 & 274.0 & 56.0 \\
\hline $7 \mathrm{R}-2,42 \mathrm{~cm}$ (Piece 6) & 66.49 & $\mathrm{HZ}$ & vein netwk & i & 000 & 0 & & \\
\hline 7R-2, $67 \mathrm{~cm}$ (Piece 9) & 66.74 & TROCT & vein & 1 & 170 & 80 & & \\
\hline $7 \mathrm{R}-2,97 \mathrm{~cm}$ (Piece 12) & 67.04 & $\mathrm{HZ}$ & foliation & 1 & 305 & 72 & 118.2 & 50.0 \\
\hline $7 \mathrm{R}-2,112 \mathrm{~cm}$ (Piece 13) & 67.19 & $\mathrm{HZ}$ & foliation & i & 040 & 86 & 184.0 & 54.0 \\
\hline $7 \mathrm{R}-2,132 \mathrm{~cm}$ (Piece 16) & 67.39 & $\mathrm{HZ}$ & foliation & i & 145 & 73 & & \\
\hline $7 \mathrm{R}-3,12 \mathrm{~cm}$ (Piece 2) & 67.65 & $\mathrm{HZ}$ & foliation & 1 & 050 & 87 & & \\
\hline $8 \mathrm{R}-1,39 \mathrm{~cm}$ (Piece 6) & 74.69 & $\mathrm{HZ}$ & foliation & i & 150 & 81 & & \\
\hline $8 \mathrm{R}-1,44 \mathrm{~cm}$ (Piece 7) & 74.74 & $\mathrm{HZ}$ & vein & I & 011 & 25 & -040.5 & 49.1 \\
\hline $8 \mathrm{R}-1,48 \mathrm{~cm}$ (Piece 7) & 74.78 & $\mathrm{HZ}$ & foliation & 2 & 200 & 37 & -040.5 & 49.1 \\
\hline $8 \mathrm{R}-1,71 \mathrm{~cm}$ (Piece 10) & 75.01 & TROCT & ign contact & 1 & 064 & 22 & & \\
\hline $8 \mathrm{R}-2,26 \mathrm{~cm}$ (Piece 3$)$ & 75.94 & $\mathrm{G}$ & foln in G & 1 & 010 & 70 & & \\
\hline $9 \mathrm{R}-1.78 \mathrm{~cm}$ (Piece 12) & 84.78 & $\mathrm{HZ}$ & vein netwk & 1 & 025 & 32 & 094.0 & 16.0 \\
\hline 9R-1, $117 \mathrm{~cm}$ (Piece 18) & 85.17 & DN & vein & 1 & 355 & 45 & 052.1 & -58.9 \\
\hline $9 \mathrm{R}-1,115 \mathrm{~cm}$ (Piece 18) & 85.15 & DN & vein & 2 & 203 & 42 & 052.1 & -58.9 \\
\hline $9 \mathrm{R}-1,125 \mathrm{~cm}$ (Piece 19) & 85.25 & $\mathrm{DN}$ & vein & 1 & 028 & 53 & 223.0 & 35.0 \\
\hline $9 \mathrm{R}-1,128 \mathrm{~cm}$ (Piece 19) & 85.28 & $\mathrm{DN}$ & vein netwk & 2 & 305 & 17 & 223.0 & 35.0 \\
\hline $9 \mathrm{R}-1,126 \mathrm{~cm}$ (Piece 19) & 85.26 & DN & vein & 3 & 267 & 47 & 223.0 & 35.0 \\
\hline 9R-1, $147 \mathrm{~cm}$ (Piece 22) & 85.47 & DN & vein & 1 & 358 & 90 & & \\
\hline $9 \mathrm{R}-1,147 \mathrm{~cm}$ (Piece 22) & 85.47 & DN & vein netwk & 2 & 208 & 74 & & \\
\hline 9R-1, $144 \mathrm{~cm}$ (Piece 22) & 85.44 & DN & vein & 3 & 060 & 28 & & \\
\hline $10 \mathrm{~W}-1,75 \mathrm{~cm}$ (Piece 9) & 94.45 & TROCT & dykelet & 1 & 000 & 80 & & \\
\hline Hole 895E- & & & & & & & & \\
\hline IR-1, $20 \mathrm{~cm}$ (Piece 3) & 0.20 & ROD G & vein & 1 & 039 & 26 & & \\
\hline IR-1, $68 \mathrm{~cm}$ (Piece 7) & 0.68 & ROD G & vein & 1 & 205 & 44 & & \\
\hline IR-1, $87 \mathrm{~cm}$ (Piece 9) & 0.87 & ROD G & vein & 1 & 199 & 15 & & \\
\hline $1 \mathrm{R}-\mathrm{I}, 107 \mathrm{~cm}$ (Piece 12) & 1.07 & ROD G & vein & 1 & 092 & 48 & & \\
\hline 1R-1, $137 \mathrm{~cm}$ (Piece 16) & 1.37 & ROD G & vein & 1 & 150 & 19 & & \\
\hline IR-2, $25 \mathrm{~cm}$ (Piece 5) & 1.69 & DN & vein & 1 & 178 & 10 & & \\
\hline $1 \mathrm{R}-2,70 \mathrm{~cm}$ (Piece 15 ) & 2.14 & DN & vein & 1 & 344 & 44 & -073.5 & 46.7 \\
\hline $1 \mathrm{R}-2,73 \mathrm{~cm}$ (Piece 15) & 2.17 & $\mathrm{DN}$ & vein & 2 & 316 & 40 & -073.5 & 46.7 \\
\hline IR-2, $78 \mathrm{~cm}$ (Piece 15) & 2.22 & DN & vein & 3 & 085 & 29 & -073.5 & 46.7 \\
\hline IR-2, $82 \mathrm{~cm}$ (Piece 15) & 2.26 & DN & vein & 4 & 128 & 18 & -073.5 & 46.7 \\
\hline $1 \mathrm{R}-2,98 \mathrm{~cm}$ (Piece 18) & 2.42 & $\mathrm{DN}$ & vein & 1 & 120 & 23 & 318.0 & 43.0 \\
\hline
\end{tabular}


Table 2 (continued).

\begin{tabular}{|c|c|c|c|c|c|c|c|c|}
\hline & Depth & & & & Con & ned & Stable $\mathrm{m}$ & g. rem. \\
\hline Sample number & (mbsf) & Lith. & Feature type & Feat. \# & $\operatorname{str}\left({ }^{\circ}\right)$ & $\operatorname{dip}\left({ }^{\circ}\right)$ & $\operatorname{dec}\left({ }^{\circ}\right)$ & $\operatorname{inc}\left({ }^{\circ}\right)$ \\
\hline IR-2, $107 \mathrm{~cm}$ (Piece 18) & 2.51 & DN & vein & 2 & 275 & 86 & 318.0 & 43.0 \\
\hline IR-2, $109 \mathrm{~cm}$ (Piece 18) & 2.53 & DN & vein & 3 & 270 & 90 & 318.0 & 43.0 \\
\hline IR-2, $107 \mathrm{~cm}$ (Piece 18) & 2.51 & DN & vein & 4 & 071 & 52 & 318.0 & 43.0 \\
\hline $1 \mathrm{R}-2,114 \mathrm{~cm}$ (Piece 18) & 2.58 & DN & vein & 5 & 069 & 52 & 318.0 & 43.0 \\
\hline $1 \mathrm{R}-2,116 \mathrm{~cm}$ (Piece 18) & 2.60 & DN & vein & 6 & 270 & 90 & 318.0 & 43.0 \\
\hline $1 \mathrm{R}-2,119 \mathrm{~cm}$ (Piece 18) & 2.63 & DN & vein & 7 & 125 & 46 & 318.0 & 43.0 \\
\hline IR-2, $130 \mathrm{~cm}$ (Piece 18) & 2.74 & DN & vein & 8 & 270 & 90 & 318.0 & 43.0 \\
\hline IR-2, $132 \mathrm{~cm}$ (Piece 18) & 2.76 & DN & vein & 9 & 335 & 65 & 318.0 & 43.0 \\
\hline IR-2, $102 \mathrm{~cm}$ (Piece 18) & 2.46 & DN & vein & 10 & 304 & 42 & 318.0 & 43.0 \\
\hline IR-3, $10 \mathrm{~cm}$ (Piece 1) & 2.96 & DN & foliation & 1 & 283 & 39 & & \\
\hline IR-3, $24 \mathrm{~cm}$ (Piece 2) & 3.10 & DN & vein & i & 005 & 45 & 317.0 & 31.0 \\
\hline IR-3, $28 \mathrm{~cm}$ (Piece 2) & 3.14 & DN & vein netwk & 2 & 141 & 13 & 317.0 & 31.0 \\
\hline 1R-3, $23 \mathrm{~cm}$ (Piece 2) & 3.09 & $\mathrm{DN} / \mathrm{HZ}$ & ign contact & 3 & 040 & 57 & 317.0 & 31.0 \\
\hline IR-3, $60 \mathrm{~cm}$ (Piece 4) & 3.46 & DN & vein netwk & 1 & 045 & 3 & 151.4 & 37.8 \\
\hline IR-3, $50 \mathrm{~cm}$ (Piece 4) & 3.36 & DN & foliation & 3 & 065 & 41 & 151.4 & 37.8 \\
\hline $1 \mathrm{R}-3,70 \mathrm{~cm}$ (Piece 4) & 3.56 & DN & vein & 2 & 000 & 90 & 151.4 & 37.8 \\
\hline IR-3,99 cm (Piece 5) & 3.85 & $\mathrm{HZ}$ & vein netwk & 1 & 218 & 31 & 253.8 & 35.0 \\
\hline IR-3, $125 \mathrm{~cm}$ (Piece 9) & 4.11 & TROCT & foliation & 1 & 000 & 40 & & \\
\hline IR-3, $135 \mathrm{~cm}$ (Piece 10) & 4.21 & TROCT & foliation & 1 & 064 & 11 & & \\
\hline IR-4, $6 \mathrm{~cm}$ (Piece 1) & 4.42 & TROCT & foliation & 1 & 000 & 25 & & \\
\hline $1 \mathrm{R}-4,16 \mathrm{~cm}$ (Piece 2) & 4.52 & TROCT & vein & i & 000 & 25 & & \\
\hline $1 \mathrm{R}-4,17 \mathrm{~cm}$ (Piece 2) & 4.53 & TROCT & foliation & 2 & 180 & 20 & & \\
\hline IR-4, $24 \mathrm{~cm}$ (Piece 3) & 4.60 & $\mathrm{DN}$ & vein netwk & 1 & 277 & 15 & & \\
\hline IR-4, $24 \mathrm{~cm}$ (Piece 3$)$ & 4.60 & DN & vein & 2 & 017 & 51 & & \\
\hline $1 \mathrm{R}-4,39 \mathrm{~cm}$ (Piece 4) & 4.75 & DN & vein & 1 & 015 & 62 & & \\
\hline $1 \mathrm{R}-4,36 \mathrm{~cm}$ (Piece 4) & 4.72 & DN & vein netwk & 2 & 315 & 7 & & \\
\hline $2 \mathrm{R}-1,86 \mathrm{~cm}$ (Piece 13) & 20.46 & ROD G & vein & 1 & 026 & 22 & & \\
\hline $2 \mathrm{R}-1,93 \mathrm{~cm}$ (Piece 14) & 20.53 & ROD G & vein & 1 & 180 & 20 & & \\
\hline 2R-1, $94 \mathrm{~cm}$ (Piece 14) & 20.54 & ROD G & vein & 2 & 315 & 50 & & \\
\hline $2 \mathrm{R}-1,106 \mathrm{~cm}$ (Piece 15) & 20.66 & ROD G & vein & 1 & 000 & 10 & & \\
\hline $2 \mathrm{R}-2,21 \mathrm{~cm}$ (Piece 2$)$ & 21.17 & $\mathrm{HZ}$ & vein netwk & 1 & 315 & 33 & 099.7 & 9.7 \\
\hline 2R-2, $59 \mathrm{~cm}$ (Piece 9) & 21.55 & DN & vein & 1 & 180 & 50 & 026.0 & 58.0 \\
\hline 2R-2, $63 \mathrm{~cm}$ (Piece 9) & 21.59 & DN & vein netwk & 2 & 135 & 7 & 026.0 & 58.0 \\
\hline $2 \mathrm{R}-2,106 \mathrm{~cm}$ (Piece 15) & 22.02 & $\mathrm{DN}$ & vein & 1 & 195 & 80 & 000.6 & 58.7 \\
\hline $2 \mathrm{R}-2,106 \mathrm{~cm}$ (Piece 15) & 22.02 & $\mathrm{DN}$ & foliation & 2 & 235 & 51 & 000.6 & 58.7 \\
\hline $3 \mathrm{R}-1,8 \mathrm{~cm}$ (Piece 2) & 29.68 & DN & vein netwk & 1 & 284 & 65 & & \\
\hline $3 \mathrm{R}-1,10 \mathrm{~cm}$ (Piece 2) & 29.70 & DN & vein & 2 & 186 & 85 & & \\
\hline $3 \mathrm{R}-1,8 \mathrm{~cm}$ (Piece 2) & 29.68 & DN & vein & 3 & 336 & 90 & & \\
\hline 3R-1, $50 \mathrm{~cm}$ (Piece 8) & 30.10 & DN & vein & 1 & 151 & 24 & & \\
\hline $3 \mathrm{R}-1,47 \mathrm{~cm}$ (Piece 8) & 30.07 & $\mathrm{DN}$ & vein & 2 & 040 & 90 & & \\
\hline $3 \mathrm{R}-1,43 \mathrm{~cm}$ (Piece 8) & 30.03 & DN & vein & 3 & 250 & 27 & & \\
\hline $3 \mathrm{R}-1,62 \mathrm{~cm}$ (Piece 10) & 30.22 & DN & vein & 1 & 060 & 79 & & \\
\hline $3 \mathrm{R}-1,59 \mathrm{~cm}$ (Piece 10) & 30.19 & DN & vein & 2 & 015 & 38 & & \\
\hline $3 \mathrm{R}-1,56 \mathrm{~cm}$ (Piece 10) & 30.16 & DN & vein & 3 & 354 & 64 & & \\
\hline 3R-1, $60 \mathrm{~cm}$ (Piece 10) & 30.20 & $\mathrm{DN}$ & foliation & 4 & 239 & 12 & & \\
\hline 3R-1, $73 \mathrm{~cm}$ (Piece 12) & 30.33 & DN & vein & 1 & 016 & 78 & & \\
\hline $3 \mathrm{R}-1,72 \mathrm{~cm}$ (Piece 12) & 30.32 & DN & vein & 2 & 192 & 82 & & \\
\hline $3 \mathrm{R}-1,80 \mathrm{~cm}$ (Piece 12) & 30.40 & $\mathrm{DN}$ & foliation & 3 & 135 & 21 & & \\
\hline 3R-1, $84 \mathrm{~cm}$ (Piece 13) & 30.44 & $\mathrm{HZ}$ & vein & 1 & 315 & 15 & & \\
\hline $3 \mathrm{R}-1,86 \mathrm{~cm}$ (Piece 13) & 30.46 & $\mathrm{HZ}$ & foliation & 2 & 324 & 24 & & \\
\hline $3 \mathrm{R}-2,2 \mathrm{~cm}$ (Piece 1) & 30.99 & DN & vein & 1 & 177 & 67 & & \\
\hline 3R-2, $10 \mathrm{~cm}$ (Piece 1) & 31.07 & DN & vein & 2 & 173 & 63 & & \\
\hline $3 \mathrm{R}-2,7 \mathrm{~cm}$ (Piece 1) & 31.04 & DN & vein netwk & 3 & 106 & 77 & & \\
\hline $3 \mathrm{R}-2,22 \mathrm{~cm}$ (Piece 3$)$ & 31.19 & DN & vein netwk & 1 & 302 & 64 & 171.0 & 46.0 \\
\hline 3R-2, $26 \mathrm{~cm}$ (Piece 3) & 31.23 & DN & vein & 2 & 256 & 4 & 171.0 & 46.0 \\
\hline 3R-2, $25 \mathrm{~cm}$ (Piece 3) & 31.22 & DN & foliation & 4 & 108 & 16 & 171.0 & 46.0 \\
\hline $3 \mathrm{R}-2,30 \mathrm{~cm}$ (Piece 3$)$ & 31.27 & DN & vein & 3 & 250 & 77 & 171.0 & 46.0 \\
\hline $3 \mathrm{R}-2,42 \mathrm{~cm}$ (Piece 4) & 31.39 & DN & vein & 1 & 199 & 62 & & \\
\hline 3R-2, $44 \mathrm{~cm}$ (Piece 4) & 31.41 & DN & vein & 2 & 205 & 90 & & \\
\hline $3 \mathrm{R}-2,38 \mathrm{~cm}$ (Piece 4) & 31.35 & DN & vein & 3 & 177 & 45 & & \\
\hline 3R-2, $40 \mathrm{~cm}$ (Piece 4) & 31.37 & $\mathrm{DN}$ & vein netwk & 4 & 294 & 47 & & \\
\hline $3 R-2,51 \mathrm{~cm}$ (Piece 5) & 31.48 & DN & vein & 1 & 050 & 64 & 151.0 & 36.8 \\
\hline 3R-2, $49 \mathrm{~cm}$ (Piece 5) & 31.46 & DN & vein & 2 & 045 & 68 & 151.0 & 36.8 \\
\hline $3 \mathrm{R}-2,54 \mathrm{~cm}$ (Piece 5) & 31.51 & DN & vein netwk & 3 & 311 & 44 & 151.0 & 36.8 \\
\hline $3 \mathrm{R}-2,60 \mathrm{~cm}$ (Piece 5) & 31.57 & DN & vein & 4 & 000 & 90 & 151.0 & 36.8 \\
\hline $3 \mathrm{R}-2,58 \mathrm{~cm}$ (Piece 5) & 31.55 & DN & vein netwk & 5 & 312 & 16 & 151.0 & 36.8 \\
\hline $3 \mathrm{R}-2,61 \cdot \mathrm{cm}$ (Piece 5) & 31.58 & $\mathrm{DN}$ & vein & 6 & 165 & 63 & 151.0 & 36.8 \\
\hline $3 \mathrm{R}-2,66 \mathrm{~cm}$ (Piece 5) & 31.63 & $\mathrm{DN}$ & vein & 7 & 171 & 54 & 151.0 & 36.8 \\
\hline $3 \mathrm{R}-2,65 \mathrm{~cm}$ (Piece 5) & 31.62 & DN & foliation & 9 & 104 & 21 & 151.0 & 36.8 \\
\hline 3R-2, $137 \mathrm{~cm}$ (Piece 14) & 32.34 & DN & vein & 1 & 355 & 90 & & \\
\hline $3 \mathrm{R}-2,136 \mathrm{~cm}$ (Piece 14) & 32.33 & $\mathrm{DN}$ & vein & 2 & 184 & 58 & & \\
\hline $3 \mathrm{R}-2,135 \mathrm{~cm}$ (Piece 14$)$ & 32.32 & DN & vein netwk & 3 & 025 & 16 & & \\
\hline 3R-3, $24 \mathrm{~cm}$ (Piece 3) & 32.71 & DN & vein & 1 & 161 & 81 & -108.0 & 44.6 \\
\hline $3 \mathrm{R}-3,37 \mathrm{~cm}$ (Piece 3 ) & 32.84 & DN & vein & 2 & 133 & 58 & -108.0 & 44.6 \\
\hline 3R-3, $30 \mathrm{~cm}$ (Piece 3$)$ & 32.77 & DN & vein netwk & 3 & 344 & 31 & -108.0 & 44.6 \\
\hline $3 \mathrm{R}-3,36 \mathrm{~cm}$ (Piece 3 ) & 32.83 & DN & foliation & 4 & 205 & 58 & -108.0 & 44.6 \\
\hline $3 \mathrm{R}-3,42 \mathrm{~cm}$ (Piece 4) & 32.89 & DN & vein & 1 & 172 & 70 & & \\
\hline 3R-3, $44 \mathrm{~cm}$ (Piece 4) & 32.91 & DN & vein netwk & 2 & 042 & 29 & & \\
\hline $3 \mathrm{R}-3,51 \mathrm{~cm}$ (Piece 5) & 32.98 & DN & vein & 1 & 199 & 76 & & \\
\hline $3 \mathrm{R}-3,56 \mathrm{~cm}$ (Piece 5) & 33.03 & DN & vein netwk & 2 & 062 & 12 & & \\
\hline $3 \mathrm{R}-3,58 \mathrm{~cm}$ (Piece 5) & 33.05 & DN & foliation & 3 & 061 & 44 & & \\
\hline 3R-3, $75 \mathrm{~cm}$ (Piece 6) & 33.22 & DN & vein & 1 & 359 & 75 & & \\
\hline $4 \mathrm{R}-1,45 \mathrm{~cm}$ (Piece 8) & 39.95 & DN & vein netwk & 1 & 039 & 27 & & \\
\hline $4 \mathrm{R}-1,43 \mathrm{~cm}$ (Piece 8) & 39.93 & DN & vein & 2 & 160 & 84 & & \\
\hline $4 \mathrm{R}-1,86 \mathrm{~cm}$ (Piece 15) & 40.36 & DN & vein & 1 & 184 & 69 & -046.5 & 30.1 \\
\hline $4 \mathrm{R}-1,88 \mathrm{~cm}$ (Piece 15) & 40.38 & DN & vein netwk & 2 & 065 & 23 & -046.5 & 30.1 \\
\hline $4 \mathrm{R}-1,96 \mathrm{~cm}$ (Piece 15) & 40.46 & DN & vein netwk & 3 & 057 & 21 & -046.5 & 30.1 \\
\hline $4 \mathrm{R}-1,98 \mathrm{~cm}$ (Piece 15) & 40.48 & DN & vein & 4 & 080 & 60 & -046.5 & 30.1 \\
\hline
\end{tabular}


Table 2 (continued).

\begin{tabular}{|c|c|c|c|c|c|c|c|c|c|c|}
\hline & Depth & & & & Con & ined & Stable m & g. rem. & & Rest ( \\
\hline Sample number & (mbsf) & Lith. & Feature type & Feat. \# & $\operatorname{str}\left(\left(^{\circ}\right)\right.$ & $\operatorname{dip}\left({ }^{\circ}\right)$ & $\operatorname{dec}\left(\left(^{\circ}\right)\right.$ & $\operatorname{inc}\left({ }^{\circ}\right)$ & $\begin{array}{c}\text { Meas. } \\
\text { by }\end{array}$ & $\operatorname{str}\left({ }^{\circ}\right)$ \\
\hline $4 \mathrm{R}-1,119 \mathrm{~cm}$ (Piece 17) & 40.69 & DN & vein netwk & 1 & 199 & 26 & & & & \\
\hline $4 \mathrm{R}-1,121 \mathrm{~cm}$ (Piece 17) & 40.71 & DN & vein netwk & 2 & 240 & 25 & & & & \\
\hline $4 \mathrm{R}-2,23 \mathrm{~cm}$ (Piece 2 ) & 41.17 & DN & vein & 1 & 235 & 65 & 035.8 & 22.5 & J & 199.2 \\
\hline $4 \mathrm{R}-2,18 \mathrm{~cm}$ (Piece 2) & 41.12 & DN & foliation & 2 & 017 & 56 & 035.8 & 22.5 & $\mathrm{~J}$ & 341.2 \\
\hline 4R-2, $55 \mathrm{~cm}$ (Piece 4) & 41.49 & DN & vein & 2 & 205 & 76 & 068.0 & 40.0 & C & 137.0 \\
\hline 4R-2, $78 \mathrm{~cm}$ (Piece 8) & 41.72 & DN & vein & 1 & 007 & 70 & & & & \\
\hline $4 \mathrm{R}-2,80 \mathrm{~cm}$ (Piece 8 ) & 41.74 & IMP DN & plag impr & 2 & 253 & 14 & & & & \\
\hline $4 \mathrm{R}-2,97 \mathrm{~cm}$ (Piece 10) & 41.91 & DN & vein & 1 & 060 & 32 & & & & \\
\hline $4 \mathrm{R}-2,123 \mathrm{~cm}$ (Piece 14) & 42.17 & DN & vein & 1 & 019 & 64 & & & & \\
\hline $4 \mathrm{R}-2,127 \mathrm{~cm}$ (Piece 14) & 42.21 & DN & vein & 2 & 163 & 44 & & & & \\
\hline 4R-2, $126 \mathrm{~cm}$ (Piece 14) & 42.20 & DN & vein netwk & 3 & 255 & 44 & & & & \\
\hline $\begin{array}{l}5 \mathrm{R}-1,88 \mathrm{~cm} \text { (Piece 16) } \\
5 \mathrm{R}-\mathrm{I}, 105 \mathrm{~cm} \text { (Piece 18) }\end{array}$ & $\begin{array}{l}49.78 \\
49.95\end{array}$ & $\begin{array}{l}\text { DN } \\
\text { DN }\end{array}$ & $\begin{array}{l}\text { vein } \\
\text { vein }\end{array}$ & $\begin{array}{l}1 \\
1\end{array}$ & $\begin{array}{l}163 \\
002\end{array}$ & $\begin{array}{l}86 \\
35\end{array}$ & 251.5 & 32.6 & $\mathbf{J}$ & 110.5 \\
\hline $5 \mathrm{R}-1,104 \mathrm{~cm}$ (Piece 18) & 49.94 & DN & vein & 3 & 038 & 70 & 251.5 & 32.6 & $\mathrm{~J}$ & 146.5 \\
\hline $5 \mathrm{R}-1,148 \mathrm{~cm}$ (Piece 21) & 50.38 & DN & vein & 1 & 000 & 47 & -143.4 & 44.6 & $\mathrm{~S}$ & 143.4 \\
\hline $5 \mathrm{R}-1,132 \mathrm{~cm}$ (Piece 21) & 50.22 & DN & vein & 2 & 315 & 65 & -143.4 & 44.6 & $\mathrm{~S}$ & 098.4 \\
\hline $5 \mathrm{R}-1,142 \mathrm{~cm}$ (Piece 21) & 50.32 & DN & vein & 3 & 024 & 69 & -143.4 & 44.6 & $\mathrm{~S}$ & 167.4 \\
\hline $5 \mathrm{R}-1,141 \mathrm{~cm}$ (Piece 21) & 50.31 & DN & vein netwk & 4 & 275 & 40 & -143.4 & 44.6 & $\mathrm{~S}$ & 058.4 \\
\hline $5 R-1,133 \mathrm{~cm}$ (Piece 21) & 50.23 & DN & vein & 5 & 356 & 82 & -143.4 & 44.6 & $\mathrm{~S}$ & 139.4 \\
\hline $5 \mathrm{R}-1,140 \mathrm{~cm}$ (Piece 21) & 50.30 & DN & foliation & 6 & 218 & 50 & -143.4 & 44.6 & $\mathrm{~s}$ & 001.4 \\
\hline $5 \mathrm{R}-2,45 \mathrm{~cm}$ (Piece 9) & 50.85 & DN & vein & 1 & 018 & 66 & 130.0 & 38.0 & $\mathrm{P}$ & 248.0 \\
\hline $5 \mathrm{R}-2,43 \mathrm{~cm}$ (Piece 9) & 50.83 & DN & vein & 2 & 016 & 71 & 130.0 & 38.0 & $\mathrm{P}$ & 246.0 \\
\hline $5 \mathrm{R}-2,52 \mathrm{~cm}$ (Piece 9) & 50.92 & DN & foliation & 3 & 132 & 42 & 130.0 & 38.0 & $\mathrm{P}$ & 002.0 \\
\hline $5 \mathrm{R}-2,92 \mathrm{~cm}$ (Piece 15) & 51.32 & DN & vein & 1 & 220 & 71 & & & & \\
\hline $5 \mathrm{R}-2,89 \mathrm{~cm}$ (Piece 15) & 51.29 & DN & vein & 2 & 025 & 35 & & & & \\
\hline $5 \mathrm{R}-2,93 \mathrm{~cm}$ (Piece 15$)$ & 51.33 & DN & vein & 3 & 018 & 34 & & & & \\
\hline $5 \mathrm{R}-2,116 \mathrm{~cm}$ (Piece 18) & 51.56 & DN & vein & 1 & 166 & 72 & -073.8 & 27.1 & $S$ & 239.8 \\
\hline $5 \mathrm{R}-2,113 \mathrm{~cm}$ (Piece 18) & 51.53 & DN & vein & 2 & 040 & 49 & -073.8 & 27.1 & $\mathrm{~S}$ & 113.8 \\
\hline $5 \mathrm{R}-2,108 \mathrm{~cm}$ (Piece 18) & 51.48 & DN & vein & 3 & 030 & 35 & -073.8 & 27.1 & $\mathrm{~S}$ & 103.8 \\
\hline $5 \mathrm{R}-2,137 \mathrm{~cm}$ (Piece 20) & 51.77 & DN & vein & 1 & 033 & 45 & 292.0 & 39.0 & $\mathrm{C}$ & 101.0 \\
\hline SR-2, $144 \mathrm{~cm}$ (Piece 20) & 51.84 & DN & vein & 2 & 051 & 21 & 292.0 & 39.0 & $\mathrm{C}$ & 119.0 \\
\hline $5 \mathrm{R}-3,7 \mathrm{~cm}$ (Piece 1) & 51.97 & DN & vein & 1 & 180 & 30 & & & & \\
\hline $5 \mathrm{R}-3,15 \mathrm{~cm}$ (Piece 1) & 52.05 & DN & vein netwk & 2 & 000 & 50 & & & & \\
\hline SR-3, I cm (Piece 1) & 51.91 & DN & vein & 3 & 350 & 40 & & & & \\
\hline $5 \mathrm{R}-3,23 \mathrm{~cm}$ (Piece 2$)$ & 52.13 & DN & vein & 1 & 180 & 60 & 044.7 & 32.1 & $\mathrm{~J}$ & 135.3 \\
\hline $5 \mathrm{R}-3,20 \mathrm{~cm}$ (Piece 2) & 52.10 & DN & vein & 2 & 079 & 25 & 044.7 & 32.1 & J & 034.3 \\
\hline $5 \mathrm{R}-3,22 \mathrm{~cm}$ (Piece 2) & 52.12 & DN & foliation & 3 & 028 & 46 & 044.7 & 32.1 & J & 343.3 \\
\hline $5 \mathrm{R}-3,55 \mathrm{~cm}$ (Piece 6) & 52.45 & DN & vein & 1 & 180 & 45 & & & & \\
\hline $5 \mathrm{R}-3,69 \mathrm{~cm}$ (Piece 8) & 52.59 & DN & vein netwk & 1 & 185 & 50 & & & & \\
\hline $6 \mathrm{R}-1,58 \mathrm{~cm}$ (Piece 11) & 59.18 & DN & vein & 1 & 170 & 55 & & & & \\
\hline $6 \mathrm{R}-1,113 \mathrm{~cm}$ (Piece 21) & 59.73 & DN & vein & 1 & 154 & 22 & & & & \\
\hline $\begin{array}{l}\text { 6R-1, } 124 \mathrm{~cm} \text { (Piece 23) } \\
6 \mathrm{R}-2,13 \mathrm{~cm} \text { (Piece 3) }\end{array}$ & $\begin{array}{l}59.84 \\
60.23\end{array}$ & $\begin{array}{l}\text { DN } \\
\text { DN }\end{array}$ & $\begin{array}{l}\text { vein } \\
\text { vein }\end{array}$ & $\begin{array}{l}1 \\
1\end{array}$ & $\begin{array}{l}210 \\
151\end{array}$ & $\begin{array}{l}86 \\
26\end{array}$ & 106.0 & 11.0 & C & 104.0 \\
\hline $6 \mathrm{R}-2,18 \mathrm{~cm}$ (Piece 3 ) & 60.28 & DN & vein & 2 & 121 & 52 & & & & \\
\hline $6 \mathrm{R}-2,31 \mathrm{~cm}$ (Piece 4) & 60.41 & $\mathrm{DN}$ & vein & $i$ & 084 & 40 & 173.0 & 18.0 & $\mathrm{C}$ & 271.0 \\
\hline $6 \mathrm{R}-2,25 \mathrm{~cm}$ (Piece 4) & 60.35 & DN & vein & 2 & 320 & 42 & 173.0 & 18.0 & $\mathrm{C}$ & 147.0 \\
\hline $6 \mathrm{R}-2,27 \mathrm{~cm}$ (Piece 4) & 60.37 & $\mathrm{DN}$ & vein & 3 & 310 & 57 & 173.0 & 18.0 & C & 137.0 \\
\hline $6 \mathrm{R}-2,21 \mathrm{~cm}$ (Piece 5) & 60.31 & DN & vein & 1 & 084 & 40 & 083.0 & 15.6 & $\mathrm{~J}$ & 001.0 \\
\hline $6 \mathrm{R}-2,24 \mathrm{~cm}$ (Piece 5) & 60.34 & DN & vein & 2 & 310 & 42 & 083.0 & 15.6 & J & 227.0 \\
\hline $6 \mathrm{R}-2,27 \mathrm{~cm}$ (Piece 5) & 60.37 & DN & vein & 3 & 310 & 57 & 083.0 & 15.6 & $\mathrm{~J}$ & 227.0 \\
\hline 6R-2, $39 \mathrm{~cm}$ (Piece 5) & 60.49 & DN & vein & 4 & 180 & 80 & 083.0 & 15.6 & $\mathbf{J}$ & 097.0 \\
\hline $6 \mathrm{R}-2,52 \mathrm{~cm}$ (Piece 5) & 60.62 & $\mathrm{DN}$ & vein & 5 & 045 & 7 & 083.0 & 15.6 & J & 322.0 \\
\hline $6 \mathrm{R}-2,58 \mathrm{~cm}$ (Piece 5$)$ & 60.68 & DN & vein & 6 & 176 & 50 & 083.0 & 15.6 & J & 093.0 \\
\hline 6R-2, $64 \mathrm{~cm}$ (Piece 5) & 60.74 & DN & vein & 7 & 000 & 15 & 083.0 & 15.6 & $\mathrm{~J}$ & 277.0 \\
\hline $6 \mathrm{R}-2,68 \mathrm{~cm}$ (Piece 5) & 60.78 & DN & vein & 8 & 225 & 86 & 083.0 & 15.6 & $\mathrm{~J}$ & 142.0 \\
\hline 6R-2, $87 \mathrm{~cm}$ (Piece 5) & 60.97 & $\mathrm{DN}$ & vein & 9 & 072 & 16 & 083.0 & 15.6 & J & 349.0 \\
\hline $6 \mathrm{R}-2,90 \mathrm{~cm}$ (Piece 5) & 61.00 & $\mathrm{DN}$ & vein & 10 & 325 & 86 & 083.0 & 15.6 & J & 242.0 \\
\hline 6R-2, $80 \mathrm{~cm}$ (Piece 5) & 60.90 & DN & foliation & 11 & 150 & 68 & 083.0 & 15.6 & $\mathbf{J}$ & 067.0 \\
\hline $6 \mathrm{R}-2,103 \mathrm{~cm}$ (Piece 6) & 61.13 & DN & vein & 1 & 005 & 55 & & & & \\
\hline $6 \mathrm{R}-2,106 \mathrm{~cm}$ (Piece 7) & 61.16 & $\mathrm{DN}$ & vein & i & 210 & 68 & 050.3 & 11.2 & & 159.7 \\
\hline $6 \mathrm{R}-2,110 \mathrm{~cm}$ (Piece 7) & 61.20 & DN & vein & 2 & 197 & 65 & 050.3 & 11.2 & & 146.7 \\
\hline $6 \mathrm{R}-3,5 \mathrm{~cm}$ (Piece 1) & 61.36 & $\mathrm{DN}$ & vein & 1 & 191 & 25 & 216.1 & 4.5 & J & 334.9 \\
\hline $6 \mathrm{R}-3,30 \mathrm{~cm}$ (Piece 1) & 61.61 & DN & vein & 2 & 225 & 14 & 216.1 & 4.5 & J & 008.9 \\
\hline $6 \mathrm{R}-3,38 \mathrm{~cm}$ (Piece 1) & 61.69 & DN & vein & 3 & 180 & 25 & 216.1 & 4.5 & J & 323.9 \\
\hline $6 \mathrm{R}-3,6 \mathrm{~cm}$ (Piece 1) & 61.37 & DN & vein & 4 & 162 & 41 & 216.1 & 4.5 & $\mathbf{J}$ & 305.9 \\
\hline $6 \mathrm{R}-3,40 \mathrm{~cm}$ (Piece 1) & 61.71 & DN & vein & 5 & 184 & 40 & 216.1 & 4.5 & J & 327.9 \\
\hline $6 \mathrm{R}-3,31 \mathrm{~cm}$ (Piece 1) & 61.62 & $\mathrm{DN}$ & vein & 6 & 180 & 80 & 216.1 & 4.5 & J & 323.9 \\
\hline $6 \mathrm{R}-3,10 \mathrm{~cm}$ (Piece 1) & 61.41 & DN & foliation & 7 & 095 & 88 & 216.1 & 4.5 & $\mathbf{J}$ & 238.9 \\
\hline $6 \mathrm{R}-3,48 \mathrm{~cm}$ (Piece 2) & 61.79 & $\mathrm{DN}$ & vein & 1 & 291 & 67 & 211.0 & 13.0 & $\mathrm{C}$ & 080.0 \\
\hline $6 \mathrm{R}-3,53 \mathrm{~cm}$ (Piece 2) & 61.84 & DN & vein & 2 & 225 & 55 & 211.0 & 13.0 & $\mathrm{C}$ & 014.0 \\
\hline 6R-3, $57 \mathrm{~cm}$ (Piece 2) & 61.88 & DN & vein & 3 & 315 & 90 & 211.0 & 13.0 & $\mathrm{C}$ & 104.0 \\
\hline $6 \mathrm{R}-3,65 \mathrm{~cm}$ (Piece 2) & 61.96 & $\mathrm{DN}$ & vein & 4 & 313 & 72 & 211.0 & 13.0 & $\mathrm{C}$ & 102.0 \\
\hline $6 \mathrm{R}-3,53 \mathrm{~cm}$ (Piece 2) & 61.84 & $\mathrm{DN}$ & vein & 6 & 076 & 20 & 211.0 & 13.0 & $\mathrm{C}$ & 225.0 \\
\hline $6 \mathrm{R}-3,55 \mathrm{~cm}$ (Piece 2) & 61.86 & DN & vein & 7 & 212 & 34 & 211.0 & 13.0 & $\mathrm{C}$ & 001.0 \\
\hline $6 \mathrm{R}-3,80 \mathrm{~cm}$ (Piece 4) & 62.11 & DN & vein & 1 & 030 & 90 & & & & \\
\hline $6 \mathrm{R}-3,79 \mathrm{~cm}$ (Piece 4) & 62.10 & DN & vein & 2 & 276 & 40 & & & & \\
\hline $6 \mathrm{R}-3,94 \mathrm{~cm}$ (Piece 5) & 62.25 & DN & vein & 1 & 165 & 26 & -026.8 & 57.6 & $\mathrm{~S}$ & 191.8 \\
\hline 6R-3, $98 \mathrm{~cm}$ (Piece 5) & 62.29 & DN & vein & 2 & 210 & 30 & -026.8 & 57.6 & $\mathrm{~S}$ & 236.8 \\
\hline $6 \mathrm{R}-3,103 \mathrm{~cm}$ (Piece 5) & 62.34 & DN & vein & 3 & 225 & 50 & -026.8 & 57.6 & $\mathrm{~S}$ & 251.8 \\
\hline $6 \mathrm{R}-3,107 \mathrm{~cm}$ (Piece 5) & 62.38 & $\mathrm{DN}$ & vein & 4 & 195 & 37 & -026.8 & 57.6 & $\mathrm{~S}$ & 221.8 \\
\hline $6 \mathrm{R}-3,87 \mathrm{~cm}$ (Piece 5$)$ & 62.18 & DN & vein & 5 & 172 & 17 & -026.8 & 57.6 & $\mathrm{~S}$ & 198.8 \\
\hline $6 \mathrm{R}-3,88 \mathrm{~cm}$ (Piece 5) & 62.19 & $\mathrm{DN}$ & vein & 6 & 195 & 38 & -026.8 & 57.6 & $\mathrm{~S}$ & 221.8 \\
\hline 6R-3,89 cm (Piece 5) & 62.20 & DN & foliation & 7 & 000 & 70 & -026.8 & 57.6 & $\mathrm{~s}$ & 026.8 \\
\hline $6 \mathrm{R}-4,2 \mathrm{~cm}$ (Piece 1) & 62.44 & DN & vein & 1 & 335 & 32 & & & & \\
\hline $6 \mathrm{R}-4,16 \mathrm{~cm}$ (Piece 1) & 62.58 & DN & vein & 2 & 355 & 60 & & & & \\
\hline $6 \mathrm{R}-4,28 \mathrm{~cm}$ (Piece 1) & 62.70 & DN & vein & 3 & 200 & 85 & & & & \\
\hline $6 \mathrm{R}-4,12 \mathrm{~cm}$ (Piece 1) & 62.54 & DN & foliation & 4 & 300 & 63 & & & & \\
\hline $6 \mathrm{R}-4,41 \mathrm{~cm}$ (Piece 2) & 62.83 & DN & vein & 1 & 180 & 25 & -064.5 & 35.5 & $\mathbf{J}$ & 244.5 \\
\hline
\end{tabular}


Table 2 (continued).

\begin{tabular}{|c|c|c|c|c|c|c|c|c|}
\hline & Depth & & & & Con & ined & Stable n & ag. rem. \\
\hline Sample number & (mbsf) & Lith. & Feature type & Feat. \# & $\operatorname{str}\left({ }^{\circ}\right)$ & $\operatorname{dip}\left({ }^{\circ}\right)$ & $\operatorname{dec}\left({ }^{\circ}\right)$ & $\operatorname{inc}\left({ }^{\circ}\right)$ \\
\hline $6 \mathrm{R}-4,45 \mathrm{~cm}$ (Piece 2) & 62.87 & DN & vein & 2 & 195 & 71 & -064.5 & 35.5 \\
\hline $6 \mathrm{R}-4,57 \mathrm{~cm}$ (Piece 2 ) & 62.99 & DN & vein & 3 & 160 & 62 & -064.5 & 35.5 \\
\hline $6 \mathrm{R}-4,62 \mathrm{~cm}$ (Piece 2) & 63.04 & DN & vein & 4 & 000 & 20 & -064.5 & 35.5 \\
\hline $6 \mathrm{R}-4,65 \mathrm{~cm}$ (Piece 2) & 63.07 & DN & vein & 5 & 170 & 20 & -064.5 & 35.5 \\
\hline $6 \mathrm{R}-4,70 \mathrm{~cm}$ (Piece 2$)$ & 63.12 & DN & vein & 6 & 346 & 21 & -064.5 & 35.5 \\
\hline $6 \mathrm{R}-4,51 \mathrm{~cm}$ (Piece 2) & 62.93 & DN & foliation & 7 & 276 & 80 & -064.5 & 35.5 \\
\hline $6 \mathrm{R}-4,106 \mathrm{~cm}$ (Piece 6) & 63.48 & DN & vein & 1 & 180 & 40 & & \\
\hline $6 \mathrm{R}-4,109 \mathrm{~cm}$ (Piece 6) & 63.51 & DN & vein & 2 & 350 & 80 & & \\
\hline $6 \mathrm{R}-4,107 \mathrm{~cm}$ (Piece 6) & 63.49 & DN & vein & 3 & 185 & 70 & & \\
\hline $6 \mathrm{R}-5,16 \mathrm{~cm}$ (Piece 1) & 63.91 & $\mathrm{DN}$ & vein & 1 & 160 & 42 & & \\
\hline $6 \mathrm{R}-5,14 \mathrm{~cm}$ (Piece 1) & 63.89 & $\mathrm{DN}$ & foliation & 2 & 274 & 75 & & \\
\hline 6R-5, $44 \mathrm{~cm}$ (Piece 4) & 64.19 & $\mathrm{DN}$ & vein & 1 & 015 & 71 & 059.0 & 38.0 \\
\hline $6 \mathrm{R}-5,70 \mathrm{~cm}$ (Piece 5) & 64.45 & DN & vein & 1 & 171 & 30 & -115.3 & 16.9 \\
\hline $6 \mathrm{R}-5,79 \mathrm{~cm}$ (Piece 5) & 64.54 & DN & vein & 2 & 190 & 85 & -115.3 & 16.9 \\
\hline 6R-5, $67 \mathrm{~cm}$ (Piece 5) & 64.42 & DN & foliation & 3 & 268 & 70 & -115.3 & 16.9 \\
\hline $6 \mathrm{R}-5,88 \mathrm{~cm}$ (Piece 6) & 64.63 & DN & vein & 1 & 170 & 80 & 203.0 & 6.0 \\
\hline 6R-5, $100 \mathrm{~cm}$ (Piece 6) & 64.75 & DN & vein & 2 & 188 & 80 & 203.0 & 6.0 \\
\hline $6 \mathrm{R}-5,95 \mathrm{~cm}$ (Piece 6) & 64.70 & DN & vein & 3 & 154 & 22 & 203.0 & 6.0 \\
\hline $6 \mathrm{R}-5,111 \mathrm{~cm}$ (Piece 6) & 64.86 & DN & vein & 4 & 175 & 45 & 203.0 & 6.0 \\
\hline $6 \mathrm{R}-5,112 \mathrm{~cm}$ (Piece 6) & 64.87 & DN & vein & 5 & 027 & 38 & 203.0 & 6.0 \\
\hline 6R-5, $92 \mathrm{~cm}$ (Piece 6) & 64.67 & DN & foliation & 6 & 265 & 84 & 203.0 & 6.0 \\
\hline $6 \mathrm{R}-6,8 \mathrm{~cm}$ (Piece 1) & 65.06 & DN & vein & 1 & 206 & 11 & & \\
\hline $6 \mathrm{R}-6,11 \mathrm{~cm}$ (Piece 1) & 65.09 & DN & foliation & 2 & 027 & 53 & & \\
\hline 6R-6, $3 \mathrm{~cm}$ (Piece 1) & 65.01 & DN & vein & 3 & 182 & 34 & & \\
\hline $6 \mathrm{R}-6,10 \mathrm{~cm}$ (Piece 1) & 65.08 & DN & vein & 4 & 198 & 9 & & \\
\hline $6 \mathrm{R}-6,8 \mathrm{~cm}$ (Piece 1) & 65.06 & DN & vein & 5 & 038 & 38 & & \\
\hline $6 \mathrm{R}-6,18 \mathrm{~cm}$ (Piece 2) & 65.16 & DN & vein & 1 & 185 & 70 & & \\
\hline 6R-6, $27 \mathrm{~cm}$ (Piece 3) & 65.25 & DN & vein & 1 & 203 & 42 & 255.2 & 13.7 \\
\hline 6R-6, $30 \mathrm{~cm}$ (Piece 3) & 65.28 & $\mathrm{DN}$ & foliation & 2 & 280 & 43 & 255.2 & 13.7 \\
\hline $7 \mathrm{R}-1,15 \mathrm{~cm}$ (Piece 2) & 68.35 & DN & vein & 1 & 002 & 45 & & \\
\hline $7 \mathrm{R}-1,11 \mathrm{~cm}$ (Piece 2) & 68.31 & DN & vein & 2 & 175 & 55 & & \\
\hline $7 \mathrm{R}-1,16 \mathrm{~cm}$ (Piece 2) & 68.36 & $\mathrm{DN}$ & foliation & 3 & 289 & 72 & & \\
\hline $7 \mathrm{R}-1,25 \mathrm{~cm}$ (Piece 3) & 68.45 & DN & vein & 1 & 015 & 71 & & \\
\hline $7 \mathrm{R}-1,49 \mathrm{~cm}$ (Piece 7) & 68.69 & $\mathrm{DN}$ & vein & 1 & 000 & 75 & & \\
\hline $7 \mathrm{R}-1,50 \mathrm{~cm}$ (Piece 7) & 68.70 & DN & vein & 2 & 205 & 85 & & \\
\hline $7 \mathrm{R}-1,62 \mathrm{~cm}$ (Piece 7) & 68.82 & DN & vein & 3 & 244 & 58 & & \\
\hline $7 \mathrm{R}-1,58 \mathrm{~cm}$ (Piece 7) & 68.78 & DN & vein & 4 & 200 & 85 & & \\
\hline $7 \mathrm{R}-1,79 \mathrm{~cm}$ (Piece 10) & 68.99 & DN & vein & 1 & 200 & 71 & 091.5 & 30.2 \\
\hline $7 \mathrm{R}-1.88 \mathrm{~cm}$ (Piece 11) & 69.08 & DN & vein & 1 & 190 & 70 & & \\
\hline $7 \mathrm{R}-1,124 \mathrm{~cm}$ (Piece 15) & 69.44 & DN & vein & 1 & 006 & 60 & 094.0 & 28.1 \\
\hline $7 \mathrm{R}-1,126 \mathrm{~cm}$ (Piece 15) & 69.46 & DN & vein & 2 & 200 & 85 & 094.0 & 28.1 \\
\hline $7 \mathrm{R}-1,135 \mathrm{~cm}$ (Piece 15) & 69.55 & DN & vein & 3 & 014 & 21 & 094.0 & 28.1 \\
\hline $7 \mathrm{R}-1,141 \mathrm{~cm}$ (Piece 15) & 69.61 & DN & vein & 4 & 340 & 52 & 094.0 & 28.1 \\
\hline $7 \mathrm{R}-1,122 \mathrm{~cm}$ (Piece 15) & 69.42 & DN & foliation & 5 & 230 & 70 & 094.0 & 28.1 \\
\hline $7 \mathrm{R}-2,5 \mathrm{~cm}$ (Piece 1) & 69.75 & $\mathrm{DN}$ & vein & 1 & 099 & 30 & & \\
\hline $7 \mathrm{R}-2,11 \mathrm{~cm}$ (Piece 1) & 69.81 & DN & foliation & 2 & 316 & 33 & & \\
\hline $7 \mathrm{R}-2,18 \mathrm{~cm}$ (Piece 2) & 69.88 & DN & vein & $i$ & 030 & 59 & & \\
\hline $7 \mathrm{R}-2,20 \mathrm{~cm}$ (Piece 2) & 69.90 & $\mathrm{DN}$ & vein & 2 & 180 & 45 & & \\
\hline $7 \mathrm{R}-2,29 \mathrm{~cm}$ (Piece 3) & 69.99 & DN & vein & 1 & 210 & 73 & & \\
\hline $7 \mathrm{R}-2,32 \mathrm{~cm}$ (Piece 3 ) & 70.02 & DN & foliation & 2 & 075 & 66 & & \\
\hline $7 \mathrm{R}-2,76 \mathrm{~cm}$ (Piece 8 ) & 70.46 & $\mathrm{HZ}$ & vein & 1 & 180 & 0 & & \\
\hline $7 \mathrm{R}-2,107 \mathrm{~cm}$ (Piece 11) & 70.77 & DN & vein & 1 & 180 & 80 & 093.8 & 29.7 \\
\hline $7 \mathrm{R}-2,110 \mathrm{~cm}$ (Piece 11) & 70.80 & DN & vein & 2 & 000 & 55 & 093.8 & 29.7 \\
\hline $7 \mathrm{R}-2,109 \mathrm{~cm}$ (Piece 11) & 70.79 & DN & foliation & 3 & 080 & 73 & 093.8 & 29.7 \\
\hline 7R-2, $132 \mathrm{~cm}$ (Piece 14) & 71.02 & DN & vein & 1 & 180 & 25 & & \\
\hline $7 \mathrm{R}-3,5 \mathrm{~cm}$ (Piece 1$)$ & 71.25 & DN & vein & i & 190 & 85 & & \\
\hline $7 \mathrm{R}-3,17 \mathrm{~cm}$ (Piece 3 ) & 71.37 & DN & vein & 1 & 205 & 58 & 106.0 & 34.0 \\
\hline $7 \mathrm{R}-3,31 \mathrm{~cm}$ (Piece 3$)$ & 71.51 & $\mathrm{DN}$ & vein & 2 & 303 & 18 & 106.0 & 34.0 \\
\hline $7 \mathrm{R}-3,46 \mathrm{~cm}$ (Piece 3 ) & 71.66 & DN & vein & 3 & 180 & 85 & 106.0 & 34.0 \\
\hline $7 \mathrm{R}-3,34 \mathrm{~cm}$ (Piece 3 ) & 71.54 & DN & foliation & 4 & 078 & 80 & 106.0 & 34.0 \\
\hline $7 \mathrm{R}-3,51 \mathrm{~cm}$ (Piece 4) & 71.71 & DN & vein & I & 005 & 15 & 019.6 & 35.4 \\
\hline 7R-3, $67 \mathrm{~cm}$ (Piece 4) & 71.87 & $\mathrm{DN}$ & vein & 2 & 135 & 7 & 019.6 & 35.4 \\
\hline 7R-3, $65 \mathrm{~cm}$ (Piece 4) & 71.85 & DN & vein netwk & 3 & 113 & 31 & 019.6 & 35.4 \\
\hline $7 \mathrm{R}-3,62 \mathrm{~cm}$ (Piece 4) & 71.82 & DN & foliation & 4 & 350 & 60 & 019.6 & 35.4 \\
\hline $7 \mathrm{R}-3,81 \mathrm{~cm}$ (Piece 6) & 72.01 & DN & vein & 1 & 040 & 62 & & \\
\hline $7 \mathrm{R}-3,78 \mathrm{~cm}$ (Piece 6) & 71.98 & $\mathrm{DN}$ & vein & 2 & 146 & 40 & & \\
\hline $7 \mathrm{R}-3,86 \mathrm{~cm}$ (Piece 6) & 72.06 & DN & vein & 3 & 195 & 80 & & \\
\hline $7 \mathrm{R}-3,115 \mathrm{~cm}$ (Piece 10) & 72.35 & DN & vein & 1 & 020 & 42 & & \\
\hline $7 \mathrm{R}-3,124 \mathrm{~cm}$ (Piece 11) & 72.44 & DN & vein & 1 & 210 & 28 & 331.0 & -10.0 \\
\hline $7 \mathrm{R}-3,129 \mathrm{~cm}$ (Piece 11) & 72.49 & DN & vein & 2 & 215 & 35 & 331.0 & -10.0 \\
\hline 7R-3, $131 \mathrm{~cm}$ (Piece 11) & 72.51 & DN & vein & 3 & 105 & 46 & 331.0 & -10.0 \\
\hline $7 \mathrm{R}-3,126 \mathrm{~cm}$ (Piece 11) & 72.46 & $\mathrm{DN}$ & foliation & 4 & 348 & 61 & 331.0 & -10.0 \\
\hline $7 \mathrm{R}-3,146 \mathrm{~cm}$ (Piece 13) & 72.66 & DN & vein & 1 & 218 & 31 & -063.8 & -13.2 \\
\hline $7 \mathrm{R}-3,145 \mathrm{~cm}$ (Piece 13) & 72.65 & $\mathrm{DN}$ & foliation & 2 & 335 & 69 & -063.8 & -13.2 \\
\hline $7 \mathrm{R}-4,10 \mathrm{~cm}$ (Piece 1) & 72.80 & $\mathrm{HZ}$ & vein & 1 & 140 & 48 & & \\
\hline $7 \mathrm{R}-4,4 \mathrm{~cm}$ (Piece 1) & 72.74 & $\mathrm{HZ}$ & foliation & 2 & 070 & 74 & & \\
\hline $7 \mathrm{R}-4,23 \mathrm{~cm}$ (Piece 3 ) & 72.93 & $\mathrm{HZ}$ & vein netwk & 1 & 318 & 57 & & \\
\hline $7 \mathrm{R}-4,35 \mathrm{~cm}$ (Piece 4) & 73.05 & $\mathrm{HZ}$ & foliation & 1 & 076 & 56 & 097.2 & 40.7 \\
\hline $7 \mathrm{R}-4,64 \mathrm{~cm}$ (Piece 5) & 73.34 & $\mathrm{HZ}$ & vein & 1 & 020 & 71 & 093.0 & 31.0 \\
\hline $7 \mathrm{R}-4,54 \mathrm{~cm}$ (Piece 5) & 73.24 & $\mathrm{HZ}$ & foliation & 2 & 298 & 38 & 093.0 & 31.0 \\
\hline $7 \mathrm{R}-4,89 \mathrm{~cm}$ (Piece 8$)$ & 73.59 & $\mathrm{HZ}$ & foliation & 1 & 156 & 62 & & \\
\hline $8 \mathrm{R}-1,37 \mathrm{~cm}$ (Piece 6) & 78.27 & DN & vein & 1 & 354 & 60 & & \\
\hline $8 \mathrm{R}-1,38 \mathrm{~cm}$ (Piece 6) & 78.28 & DN & vein & 2 & 139 & 19 & & \\
\hline $8 \mathrm{R}-1,34 \mathrm{~cm}$ (Piece 6) & 78.24 & DN & vein & 3 & 195 & 66 & & \\
\hline $8 \mathrm{R}-1,40 \mathrm{~cm}$ (Piece 6) & 78.30 & DN & vein & 4 & 354 & 56 & & \\
\hline $8 \mathrm{R}-1,29 \mathrm{~cm}$ (Piece 6) & 78.19 & DN & vein & 5 & 014 & 56 & & \\
\hline $8 \mathrm{R}-1,37 \mathrm{~cm}$ (Piece 6) & 78.27 & $\mathrm{DN}$ & foliation & 6 & 091 & 89 & & \\
\hline $8 \mathrm{R}-1,51 \mathrm{~cm}$ (Piece 8) & 78.41 & $\mathrm{DN}$ & vein & 1 & 353 & 60 & & \\
\hline
\end{tabular}


Table 2 (continued).

\begin{tabular}{|c|c|c|c|c|c|c|c|c|c|c|c|c|c|}
\hline \multirow[b]{2}{*}{ Sample number } & \multirow{2}{*}{$\begin{array}{l}\text { Depth } \\
\text { (mbsf) }\end{array}$} & \multirow[b]{2}{*}{ Lith. } & \multirow[b]{2}{*}{ Feature type } & & Con & ined & Stable m & g. rem. & & Rest ( & only) & Rest & $+1)$ \\
\hline & & & & Feat.\# & $\operatorname{str}\left({ }^{\circ}\right)$ & $\operatorname{dip}\left({ }^{\circ}\right)$ & $\operatorname{dec}\left({ }^{\circ}\right)$ & inc $\left(^{\circ}\right)$ & $\begin{array}{c}\text { Meas. } \\
\text { by }\end{array}$ & $\operatorname{str}\left({ }^{\circ}\right)$ & $\operatorname{dip}\left({ }^{\circ}\right)$ & $\operatorname{str}\left(\left(^{\circ}\right)\right.$ & $\operatorname{dip}\left({ }^{\circ}\right)$ \\
\hline $8 \mathrm{R}-1,69 \mathrm{~cm}$ (Piece 8) & 78.59 & DN & vein & 2 & 005 & 55 & & & & & & - & \\
\hline $8 \mathrm{R}-1,65 \mathrm{~cm}$ (Piece 8) & 78.55 & DN & vein & 3 & 090 & 23 & & & & & & & \\
\hline $8 \mathrm{R}-1,63 \mathrm{~cm}$ (Piece 8) & 78.53 & DN & vein & 4 & 152 & 53 & & & & & & & \\
\hline $8 \mathrm{R}-1,71 \mathrm{~cm}$ (Piece 9) & 78.61 & DN & vein & 1 & 356 & 45 & & & & & & & \\
\hline $8 \mathrm{R}-1,75 \mathrm{~cm}$ (Piece 9) & 78.65 & DN & vein netwk & 2 & 161 & 47 & & & & & & & \\
\hline $8 \mathrm{R}-1,84 \mathrm{~cm}$ (Piece 10) & 78.74 & DN & vein & 1 & 027 & 70 & & & & & & & \\
\hline $8 \mathrm{R}-1,86 \mathrm{~cm}$ (Piece 10) & 78.76 & DN & vein & 2 & 002 & 60 & & & & & & & \\
\hline $8 \mathrm{R}-1,93 \mathrm{~cm}$ (Piece 10) & 78.83 & DN & vein & 3 & 008 & 65 & & & & & & & \\
\hline $8 \mathrm{R}-1,93 \mathrm{~cm}$ (Piece 10) & 78.83 & DN & vein & 4 & 162 & 43 & & & & & & & \\
\hline $8 \mathrm{R}-1,107 \mathrm{~cm}$ (Piece 11) & 78.97 & $\mathrm{DN}$ & vein & 1 & 092 & 65 & 064.6 & 22.7 & $\mathbf{J}$ & 027.4 & 65 & 033.3 & 74.2 \\
\hline $8 \mathrm{R}-1,108 \mathrm{~cm}$ (Piece 11) & 78.98 & $\mathrm{DN}$ & vein & 2 & 164 & 46 & 064.6 & 22.7 & J & 099.4 & 46 & 097.5 & 63.9 \\
\hline $8 \mathrm{R}-1,101 \mathrm{~cm}$ (Piece 11) & 78.91 & DN & vein netwk & 3 & 253 & 57 & 064.6 & 22.7 & $J$ & 188.4 & 57 & 176.4 & 56.2 \\
\hline $8 \mathrm{R}-1,100 \mathrm{~cm}$ (Piece 11) & 78.90 & DN & foliation & 4 & 024 & 46 & 064.6 & 22.7 & J & 319.4 & 46 & 337.2 & 36.3 \\
\hline $8 \mathrm{R}-1,122 \mathrm{~cm}$ (Piece 12) & 79.12 & $\mathrm{DN}$ & vein & 1 & 163 & 64 & 045.0 & -20.0 & $\mathrm{P}(+\mathrm{C})$ & 118.0 & 64 & 128.1 & 43.2 \\
\hline $8 \mathrm{R}-1,124 \mathrm{~cm}$ (Piece 12) & 79.14 & DN & vein & 2 & 015 & 63 & 045,0 & -20.0 & $\mathrm{P}(+\mathrm{C})$ & 330.0 & 63 & 322.4 & 76.9 \\
\hline $8 \mathrm{R}-1,130 \mathrm{~cm}$ (Piece 12) & 79.20 & DN & vein & 4 & 203 & 62 & 045.0 & -20.0 & $\mathrm{P}(+\mathrm{C})$ & 158.0 & 62 & 172.7 & 55.6 \\
\hline $8 \mathrm{R}-1,129 \mathrm{~cm}$ (Piece 12) & 79.19 & DN & vein & 6 & 036 & 18 & 045.0 & -20.0 & $\mathrm{P}(+\mathrm{C})$ & 351.0 & 18 & 304.8 & 32.4 \\
\hline $8 \mathrm{R}-1,127 \mathrm{~cm}$ (Piece 12) & 79.17 & $\mathrm{DN}$ & vein & 7 & 045 & 68 & 045.0 & -20.0 & $\mathrm{P}(+\mathrm{C})$ & 000.0 & 68 & 350.5 & 70.1 \\
\hline $8 \mathrm{R}-1,125 \mathrm{~cm}$ (Piece 12) & 79.15 & DN & vein ntwk & 8 & 192 & 61 & 045.0 & -20.0 & $\mathrm{P}(+\mathrm{C})$ & 147.0 & 61 & 162.5 & 50.3 \\
\hline $8 \mathrm{R}-1,122 \mathrm{~cm}$ (Piece 12) & 79.12 & DN & foliation & 9 & 311 & 57 & 045.0 & -20.0 & $\mathrm{P}(+\mathrm{C})$ & 266.0 & 57 & 266.6 & 81.6 \\
\hline $8 \mathrm{R}-2,30 \mathrm{~cm}$ (Piece 5) & 79.69 & $\mathrm{DN}$ & vein & 1 & 288 & 56 & 170.3 & 29.6 & $\mathrm{~S}(+\mathrm{P})$ & 117.7 & 56 & 113.1 & 78.7 \\
\hline $8 \mathrm{R}-2,32 \mathrm{~cm}$ (Piece 5) & 79.71 & DN & vein & 2 & 227 & 40 & 170.3 & 29.6 & $\mathrm{~S}(+\mathrm{P})$ & 056.7 & 40 & 066.5 & 62.1 \\
\hline $8 \mathrm{R}-2,35 \mathrm{~cm}$ (Piece 5) & 79.74 & DN & vein & 3 & 056 & 58 & 170.3 & 29.6 & $S(+P)$ & 245.7 & 58 & 233.8 & 36.2 \\
\hline $8 \mathrm{R}-2,50 \mathrm{~cm}$ (Piece 5) & 79.89 & DN & vein & 4 & 062 & 75 & 170.3 & 29.6 & $\mathrm{~S}(+\mathrm{P})$ & 251.7 & 75 & 247.2 & 51.5 \\
\hline $8 \mathrm{R}-2,65 \mathrm{~cm}$ (Piece 5) & 80.04 & DN & vein & 5 & 265 & 44 & 170.3 & 29.6 & $\mathrm{~S}(+\mathrm{P})$ & 094.7 & 44 & 093.5 & 68.9 \\
\hline $8 \mathrm{R}-2,51 \mathrm{~cm}$ (Piece 5) & 79.90 & $\mathrm{DN} / \mathrm{HZ}$ & ign contact & 7 & 323 & 62 & 170.3 & 29.6 & $\mathrm{~S}(+\mathrm{P})$ & 152.7 & 62 & 144.2 & 75.3 \\
\hline $8 \mathrm{R}-2,70 \mathrm{~cm}$ (Piece 5) & 80.09 & DN & vein netwk & 8 & 241 & 50 & 170.3 & 29.6 & $\mathrm{~S}(+\mathrm{P})$ & 070.7 & 50 & 074.7 & 73.9 \\
\hline $8 \mathrm{R}-2,87 \mathrm{~cm}$ (Piece 7) & 80.26 & $\mathrm{HZ}$ & vein & 1 & 353 & 58 & 060.0 & 18.0 & C & 293.0 & 58 & 297.5 & 45.9 \\
\hline $8 \mathrm{R}-2,93 \mathrm{~cm}$ (Piece 7) & 80.32 & $\mathrm{HZ}$ & vein & 2 & 253 & 35 & 060.0 & 18.0 & C & 193.0 & 35 & 173.4 & 34.2 \\
\hline $8 \mathrm{R}-2,96 \mathrm{~cm}$ (Piece 7) & 80.35 & $\mathrm{HZ}$ & vein netwk & 3 & 184 & 50 & 060.0 & 18.0 & C & 124.0 & 50 & 119.2 & 61.4 \\
\hline $8 \mathrm{R}-2,96 \mathrm{~cm}$ (Piece 7) & 80.35 & $\mathrm{HZ}$ & foliation & 4 & 067 & 51 & 060.0 & 18.0 & C & 007.0 & 51 & 017.1 & 53.8 \\
\hline $8 \mathrm{R}-2,122 \mathrm{~cm}$ (Piece 9) & 80.61 & DN & vein & 1 & 104 & 48 & & & & & & & \\
\hline $8 \mathrm{R}-2,113 \mathrm{~cm}$ (Piece 9) & 80.52 & DN & vein & 2 & 024 & 62 & & & & & & & \\
\hline $8 \mathrm{R}-2,120 \mathrm{~cm}$ (Piece 9) & 80.59 & DN & vein netwk & 3 & 034 & 53 & & & & & & & \\
\hline $8 \mathrm{R}-3,10 \mathrm{~cm}$ (Piece 1) & 80.90 & DN & vein & 1 & 148 & 45 & -050.8 & -16.0 & $\mathrm{~J}$ & 198.8 & 45 & 214.6 & 54.4 \\
\hline 8R-3, $4 \mathrm{~cm}$ (Piece 1) & 80.84 & DN & foliation & 2 & 030 & 58 & -050.8 & -16.0 & J & 080.8 & 58 & 077.2 & 37.8 \\
\hline $8 \mathrm{R}-3,7 \mathrm{~cm}$ (Piece 1) & 80.87 & DN & vein netwk & 3 & 105 & 38 & -050.8 & -16.0 & j & 155.8 & 38 & 184.2 & 34.3 \\
\hline 8R-3, $8 \mathrm{~cm}$ (Piece 1) & 80.88 & DN & vein & 4 & 190 & 30 & -050.8 & -16.0 & J & 240.8 & 30 & 251.1 & 48.9 \\
\hline $8 \mathrm{R}-3,34 \mathrm{~cm}$ (Piece 2) & 81.14 & DN & vein & 1 & 006 & 90 & & & & & & & \\
\hline $8 \mathrm{R}-3,16 \mathrm{~cm}$ (Piece 2) & 80.96 & DN & vein & 2 & 167 & 66 & & & & & & & * \\
\hline $8 \mathrm{R}-3,25 \mathrm{~cm}$ (Piece 2) & 81.05 & $\mathrm{DN}$ & vein netwk & 4 & 195 & 44 & & & & & & & \\
\hline $8 \mathrm{R}-3,45 \mathrm{~cm}$ (Piece 3) & 81.25 & DN & vein & 1 & 123 & 18 & 061.0 & -15.0 & $\mathrm{C}$ & 062.0 & 18 & 336.9 & 9.1 \\
\hline $8 \mathrm{R}-3,47 \mathrm{~cm}$ (Piece 3) & 81.27 & DN & vein & 2 & 293 & 41 & 061.0 & -15.0 & C & 232.0 & 41 & 241.4 & 57.5 \\
\hline $8 \mathrm{R}-3,51 \mathrm{~cm}$ (Piece 3) & 81.31 & DN & vein & 3 & 080 & 77 & 061.0 & -15.0 & $\mathrm{C}$ & 019.0 & 77 & 013.6 & 71.4 \\
\hline $8 \mathrm{R}-3,56 \mathrm{~cm}$ (Piece 3) & 81.36 & DN & vein & 4 & 016 & 59 & 061.0 & -15.0 & C & 315.0 & 59 & 309.2 & 73.6 \\
\hline $8 \mathrm{R}-3,48 \mathrm{~cm}$ (Piece 3) & 81.28 & DN & vein netwk & 5 & 204 & 32 & 061.0 & -15.0 & C & 143.0 & 32 & 177.8 & 25.1 \\
\hline $8 \mathrm{R}-3,66 \mathrm{~cm}$ (Piece 5) & 81.46 & DN & vein & 1 & 072 & 56 & 141.0 & -19.0 & C & 291.0 & 56 & 287.7 & 78.3 \\
\hline $8 \mathrm{R}-3,64 \mathrm{~cm}$ (Piece 5) & 81.44 & DN & vein & 2 & 282 & 43 & 141.0 & -19.0 & C & 141.0 & 43 & 169.3 & 32.6 \\
\hline $8 \mathrm{R}-3,79 \mathrm{~cm}$ (Piece 5) & 81.59 & DN & vein & 3 & 000 & 17 & 141.0 & -19.0 & C & 219.0 & 17 & 247.6 & 36.6 \\
\hline $8 \mathrm{R}-3,86 \mathrm{~cm}$ (Piece 5) & 81.66 & DN & vein & 4 & 140 & 64 & 141.0 & -19.0 & C & 359.0 & 64 & 348.1 & 66.7 \\
\hline $8 \mathrm{R}-3,70 \mathrm{~cm}$ (Piece 5) & 81.50 & DN & vein netwk & 5 & 292 & 41 & 141.0 & -19.0 & C & 151.0 & 41 & 181.1 & 35.0 \\
\hline $8 \mathrm{R}-3,102 \mathrm{~cm}$ (Piece 6) & 81.82 & DN & vein & 1 & 354 & 40 & 040.3 & -11.0 & $\mathrm{~S}$ & 313.7 & 40 & 304.2 & 52.2 \\
\hline 8R-3, $107 \mathrm{~cm}$ (Piece 6) & 81.87 & DN & foliation & 2 & 310 & 32 & 040.3 & -11.0 & $\mathrm{~S}$ & 269.7 & 32 & 269.8 & 47.6 \\
\hline $8 \mathrm{R}-3,105 \mathrm{~cm}$ (Piece 6) & 81.85 & DN & vein netwk & 3 & 202 & 36 & 040.3 & -11.0 & $\mathrm{~S}$ & 161.7 & 36 & 184.1 & 34.0 \\
\hline $8 \mathrm{R}-3,107 \mathrm{~cm}$ (Piece 6) & 81.87 & $\mathrm{DN}$ & vein & 4 & 301 & 57 & 040.3 & -11.0 & $\mathrm{~S}$ & 260.7 & 57 & 261.8 & 72.4 \\
\hline 8R-3, $106 \mathrm{~cm}$ (Piece 6) & 81.86 & $\mathrm{DN}$ & vein & 5 & 220 & 54 & 040.3 & -11.0 & $\mathrm{~S}$ & 179.7 & 54 & 190.8 & 55.4 \\
\hline $8 \mathrm{R}-3,110 \mathrm{~cm}$ (Piece 6) & 81.90 & DN & vein & 6 & 062 & 49 & 040.3 & -11.0 & $\mathrm{~S}$ & 021.7 & 49 & 007.5 & 45.0 \\
\hline $8 \mathrm{R}-3,100 \mathrm{~cm}$ (Piece 6) & 81.80 & $\mathrm{DN}$ & vein & 7 & 303 & 18 & 040.3 & -11.0 & $\mathrm{~S}$ & 262.7 & 18 & 265.9 & 33.5 \\
\hline $8 \mathrm{R}-3,116 \mathrm{~cm}$ (Piece 7) & 81.96 & DN & vein & 1 & 190 & 80 & & & & & & & \\
\hline $8 \mathrm{R}-3,113 \mathrm{~cm}$ (Piece 7) & 81.93 & DN & vein & 2 & 100 & 45 & & & & & & & \\
\hline $8 \mathrm{R}-3,116 \mathrm{~cm}$ (Piece 7) & 81.96 & $\mathrm{DN}$ & vein & 3 & 130 & 44 & & & & & & & \\
\hline $8 \mathrm{R}-3,116 \mathrm{~cm}$ (Piece 7) & 81.96 & DN & vein netwk & 4 & 023 & 48 & & & & & & & \\
\hline $8 \mathrm{R}-4,50 \mathrm{~cm}$ (Piece 8) & 82.80 & DN & vein & 1 & 357 & 36 & & & & & & & \\
\hline $8 \mathrm{R}-4,48 \mathrm{~cm}$ (Piece 8) & 82.78 & DN & vein & 2 & 024 & 90 & & & & & & & \\
\hline $8 \mathrm{R}-4,48 \mathrm{~cm}$ (Piece 8) & 82.78 & DN & vein & 3 & 017 & 80 & & & & & & & \\
\hline $8 \mathrm{R}-4,90 \mathrm{~cm}$ (Piece 13) & 83.20 & DN & vein & 1 & 270 & 9 & & & & & & & \\
\hline $8 \mathrm{R}-4,86 \mathrm{~cm}$ (Piece 13) & 83.16 & DN & vein & 2 & 034 & 76 & & & & & & & \\
\hline $8 \mathrm{R}-4.80 \mathrm{~cm}$ (Piece 13) & 83.10 & DN & vein & 3 & 336 & 16 & & & & & & & \\
\hline $8 \mathrm{R}-4,86 \mathrm{~cm}$ (Piece 13) & 83.16 & DN & vein netwk & 4 & 304 & 28 & & & & & & & \\
\hline $8 \mathrm{R}-4,111 \mathrm{~cm}$ (Piece 17) & 83.41 & DN & vein & 1 & 188 & 55 & 029.0 & -13.0 & C & 159.0 & 55 & 172.1 & 50.5 \\
\hline $8 \mathrm{R}-4,120 \mathrm{~cm}$ (Piece 17) & 83.50 & DN & vein netwk & 2 & 201 & 42 & 029.0 & $-13,0$ & $\mathrm{C}$ & 172.0 & 42 & 191.6 & 42.6 \\
\hline $8 \mathrm{R}-4,137 \mathrm{~cm}$ (Piece 19) & 83.67 & DN & vein & 1 & 102 & 43 & & & & & & & \\
\hline $8 \mathrm{R}-4,139 \mathrm{~cm}$ (Piece 19) & 83.69 & DN & vein & 2 & 181 & 42 & & & & & & & \\
\hline $8 \mathrm{R}-4,134 \mathrm{~cm}$ (Piece 19) & 83.64 & DN & vein & 3 & 059 & 65 & & & & & & & \\
\hline $8 \mathrm{R}-4,132 \mathrm{~cm}$ (Piece 19) & 83.62 & $\mathrm{DN}$ & vein & 4 & 175 & 42 & & & & & & & \\
\hline
\end{tabular}

